GBOLOGIC LOGS FOR SBLBCTED DBEP WBLLS IN PARTS OF

OKLAHOMA, TBXAS, AND NEW MEXICO

by Scott C. Christenson, Robert B. Morton,

John S. Havens, and Roy W. Fairchild

U.S. GBOLOGICAL SURVBY

Open-File Report 86-541

Oklahoma City, Oklahoma 


\section{DEPARTMENT OF THE INTERIOR \\ DONALD PAUL HODEL, Secretary \\ U.S. GEOLOGICAL SURVEY \\ Dallas L. Peok, Director}

For additional information write to:

District Chief

U.S. Geological Survey

Water Resources Division

215 Dean A. MOGee, Room 621

Oklahoma City, OK 73102
Coples of this report can be purohased from:

U.S. Geologioal survey Books and Open-File Reports Federal Center. Box 25425

Denver, Colorado 80225 
CONTENTS

Page

Abstract.............................. 1

Introduction .......................... 1

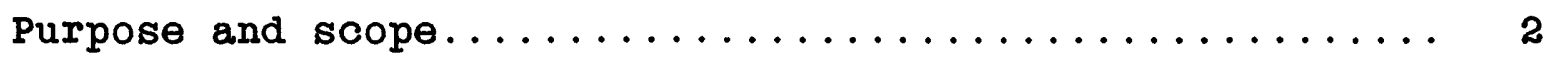

Explanation of the site-numbering system.......... 5

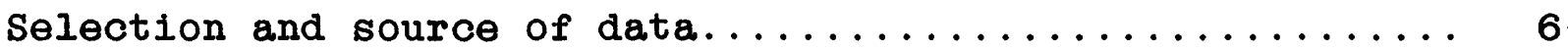

Supplemental information.................... 15

ILLUSTRATIONS

Figure 1. Map showing the location of the Central Midwest

Regional Aquifer-System Analysis study area

and the sub-project area..............4 4

2. Map showing location of wells for which geologic

logs were included in the geologic-log data

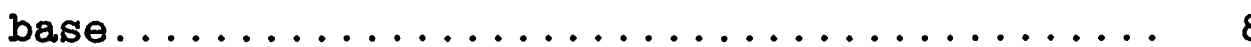

\section{TABLES}

Table 1. Chronostratigraphic units in the stratigraphic section of the Central Midwest Regional Aquifer-System Analysis study area......... 3

2. Geologic-unit codes, geologic units, and chronostratigraphic units............. 9

3. Geologic logs for wells in the study area....... 18

4. Geophysical logs for wells in the study area.... 125 


\section{CONVERSION FACTORS}

The inch-pound units of measurement given in this report may be converted to the International System (SI) of units by using the following conversion factors:

Inch-pound unit

foot ( $f t$ )

mile (m1)
Multiply by

0.3048

1.609
SI unit meter kilometer 


\title{
GEOLOGIC LOGS FOR SELECTED DEEP WELLS IN PARTS OF \\ OKLAHOMA, TEXAS, AND NEW MEXICO
}

By Scott C. Christenson, Robert B. Morton, John S. Havens, and Roy W. Fairchild

\begin{abstract}
A computerized data base of geologic logs for selected deep wells in parts of Oklahoma, Texas, and New Mexico was prepared by the U.S. Geological Survey as part of the Central Midwest Regional Aquifer-system Analysis. At least one well per county in the study area was selected, and the geologic logs for the wells were entered into the data base. Summaries of the geologic logs are presented in this report. Geophysical logs were used for interpretation of the geologic logs, and a list of these geophysical logs also is presented. These geophysical logs are available for inspection at the U.S. Geological Survey office in Oklahoma City, Oklahoma.
\end{abstract}

\section{INTRODUCTION}

The U.S. Geological Survey began a Regional Aquifer-System Analysis (RASA) program in 1978 after a congressional mandate required development of quantitative appraisals of the major aquifer systems of the United States. Twenty-elght aquifer systems which supply most of the usable ground water in the United States have been identified for this program. The Central Midwest Region Aquifer-System Analysis (CMRASA) project studies one of the these aquifer systems.

The overall purpose of the RASA program is to compile hydrologic information, to develop an linderstanding of the aquifer systems, and to develop predictive capabilities that will contribute to the effective management of the Nation's ground-water resources. The specific objectives of the program are to: (1) Assemble available geologic, hydrologic, and water-quality information, and collect additional data that may be required for definition of the aquifer system; (2) synthesize the information to support research that will contribute to a more comprehensive understanding of the flow dynamics; 3) develop, test, and establish the validity of models that simulate the hydrologic systems; (4) analyze and interpret the hydrology of the aquifer systems, and describe the hydraulic implications of alternative management practices; and (5) report the results of these studies, including the hydrologic data, the simulation models, and the final conclusions. 
The CMRASA is a study of the regional ground-water flow system in formations of Paleozoic and Mesozolic age in the central United States (table 1). The study area extends from the foothilis of the Rocky Mountains in Colorado and New Mexico to the valley of the Mississippi River in eastern Missouri, and from southern South Dakota to northern Texas (fig. 1). As part of a multistate group participating in the CMRASA, personnel of the U.S. Geological Survey in Oklahoma are responsible for studying those parts of Oklahoma, Texas, and New Mexico that are within the CMRASA study area ( $f i g .1$ ).

\section{Purpose and Scope}

The purpose of this report is to present geologic logs for selected deep wells in Oklahoma, Texas, and New Mexico that were used in the CMRASA project. No interpretive materials, such as geologic maps or cross sections, are presented in this report. The report also lists the geophysical logs that were used to interpret the geologic logs.

A large number of geologic logs are avallable for the parts of Oklahoma, Texas, and New Mexico within the study area. During the early phases of the project, a subset of geologic logs available for the study area was selected and established as a geologic-log data base. Establishing a standardized data base decreased the quantity of data, and insured that CMRASA personnel in different states used the same data base for all work. The geologic logs from at least one well per county were selected and the logs from these wells were entered into the data base. All geologic maps and cross sections that were produced as part of the CMRASA are based on data from these logs. Supplemental data in addition to the data-base logs also were used to develop the geologic maps and cross sections produced by the project.

The geologic logs are stored in a data base that is part of the Survey's National Water Data Storage and Retrieval (WATSTORE) system. The WATSTORE system is a computer data file that can be accessed by any individual or organization that is part of the Survey's National Water Data Exchange (NAWDEX) program. The stratigraphic nomenclature in WATSTORE is from many sources and may not necessarily follow the current usage of the U.S. Geological Survey. 
Table 1.--Chronostratigraphic units in the stratigraphic section of the Central Midwest Regional Aquifer-System Analysis study area

\begin{tabular}{|c|c|c|c|}
\hline Erathem & System & Series & Provincial series \\
\hline \multirow[t]{9}{*}{ Mesozoic } & \multirow[t]{3}{*}{ Cretaceous } & \multirow[t]{2}{*}{ Upper } & Gulfian \\
\hline & & & Comanchean \\
\hline & & Lower & Coahuilan \\
\hline & \multirow[t]{3}{*}{ Jurassic } & Upper & \\
\hline & & Middle & \\
\hline & & Lower & \\
\hline & \multirow[t]{3}{*}{ Triassic } & Upper & \\
\hline & & Middle & \\
\hline & & Lower & \\
\hline \multirow[t]{19}{*}{ Paleozoic } & \multirow[t]{4}{*}{ Permian } & \multirow[t]{2}{*}{ Upper } & Dchoan \\
\hline & & & Guadalupian \\
\hline & & \multirow[t]{2}{*}{ Lower } & Leonardian \\
\hline & & & Wol f campian \\
\hline & \multirow[t]{5}{*}{ Pennsy I vanian } & \multirow[t]{2}{*}{ Upper } & Virgilian \\
\hline & & & Missourian \\
\hline & & \multirow[t]{2}{*}{ Middle } & Desmoinesian \\
\hline & & & Atokan \\
\hline & & Lower & Morrowan \\
\hline & \multirow[t]{4}{*}{ Mississippian } & \multirow[t]{2}{*}{ Upper } & Chesterian \\
\hline & & & Meramecian \\
\hline & & \multirow[t]{2}{*}{ Lower } & Dsagean \\
\hline & & & Kinderhookian \\
\hline & Devonian & & \\
\hline & Silurian & & \\
\hline & \multirow[t]{3}{*}{ Ordovician } & Upper & \\
\hline & & Middle & \\
\hline & & Lower & \\
\hline & Cambrian & Upper & \\
\hline Precambrian & & & \\
\hline
\end{tabular}




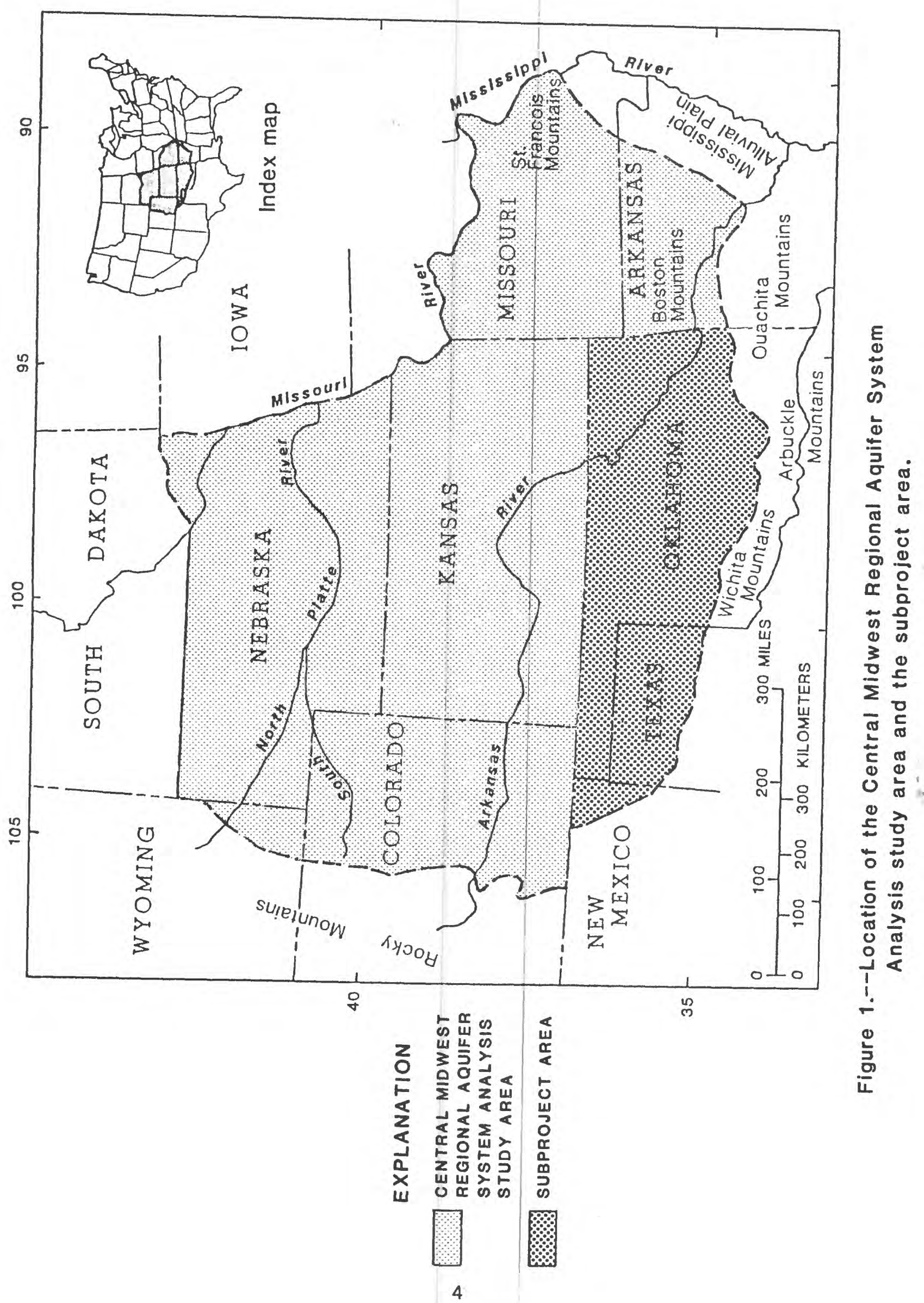




\section{Explanation of the Site-Numbering System}

The location of all data-collection sites is delineated by latitude and longitude to the nearest second. The standard system of describing a location by fractional section, section, township and range is replaced in this report by the system lllustrated in the following diagram:

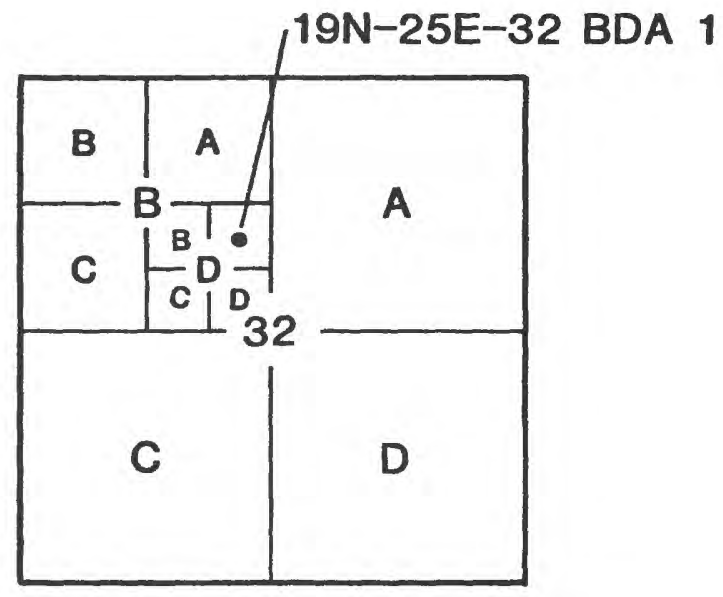

The location of the well indicated by the dot normally would be described as NE1/4 SEl/4 NWl/4, sec. 32, T. $19 \mathrm{~N} ., \mathrm{R} .25 \mathrm{E}$. The system used in this report indicates quarter-section subdivisions of the section by letters, from largest to smallest. The location of the site is 19N-25E-32 BDA 1 , and is referred to as the local number in this report. The final digit (1) is the sequential number of a well within the smallest fractional subdivision. 


\section{SELECTION AND SOURCES OF DATA}

Wells were selected for inclusion in the geologic-log data base according to the following criteria:

(1) Completeness of section --Initially, wells that had geologic logs of the most complete stratigraphic section were selected. Idealiy, the log showed the entire section between the land surface and the top of the Precambrian basement. However, this could not be achieved for some of the wells, because many geologic logs did not describe the entire depth of the well. O1I and gas companies, who may be interested only in a particular part of the stratigraphic section, produced some of the geologic logs. The logs may not include the upper part of the well, if it had been cased off. In some parts of western Oklahoma, no wells have ever penetrated to the basement. In southwestern Oklahoma, the basement may be buried under more than 40,000 feet of sedimentary rock.

(2) Quality of data -- The quality of geologic logs varied. The most accurate and complete logs that could be located for wells from each county were selected, but some less accurate and incomplete logs were used if no others were available. In counties where a geologic log that described the entire stratigraphic section was avallable, only that log was entered into the geologic-log data base. In many counties, the geologic logs from two or three wells were used to describe the complete stratigraphic section. Accurate and complete geologic logs might be avallable for the deeper parts of the section, but not the upper part. Additional geologic logs were used to describe the upper part of the section.

Geologic logs were compiled from different sources. Some geologic logs were obtained from the WATSTORE files of the U.S. Geological Survey. Additional geologic logs were obtained from commercial geologic-log distribution companies. Some geologic logs were obtained from the files of the Oklahoma City Geological Society. Finally, some of the geologic logs were prepared by the authors of this report, using geophysical logs and knowledge of the stratigraphy of the study area.

Generally, geologic logs were prepared from geophysical logs from the same wells. In some instances, geophysical logs from a nearby well were used in the preparation of the geologic log. Occasionally, the well that was selected for preparation of a geologic log did not have geophysical logs, therefore geophysical logs for a well elsewhere in the county were obtained so that at least one geophysical log would be on file for every county. Geophysical logs also were used by the 
authors to identify strata in the subsurface and to pick the top and bottom of geologic units.

The locations of each well described in this report is shown in figure 2. All wells from which geologic logs were obtained were given a geologic-unit code corresponding to the geologic unit penetrated by the well (table 2). The geologic-unit codes are the codes that are stored in the WATSTORE file.

The geologic logs are presented in table 3 , in the "Supplemental Information" section at the back of the report. The table presents the geologic units penetrated by the wells, the depths to the top and bottom of the geologic unit, and the dominant lithology. A list of geophysical logs for wells that were used to interpret the geologic logs is presented in table 4 , in the "Supplemental Information" section at the back of the report. These geophysical logs are avallable for inspection at the U.S. Geological Survey office in Oklahoma City, Oklahoma. 


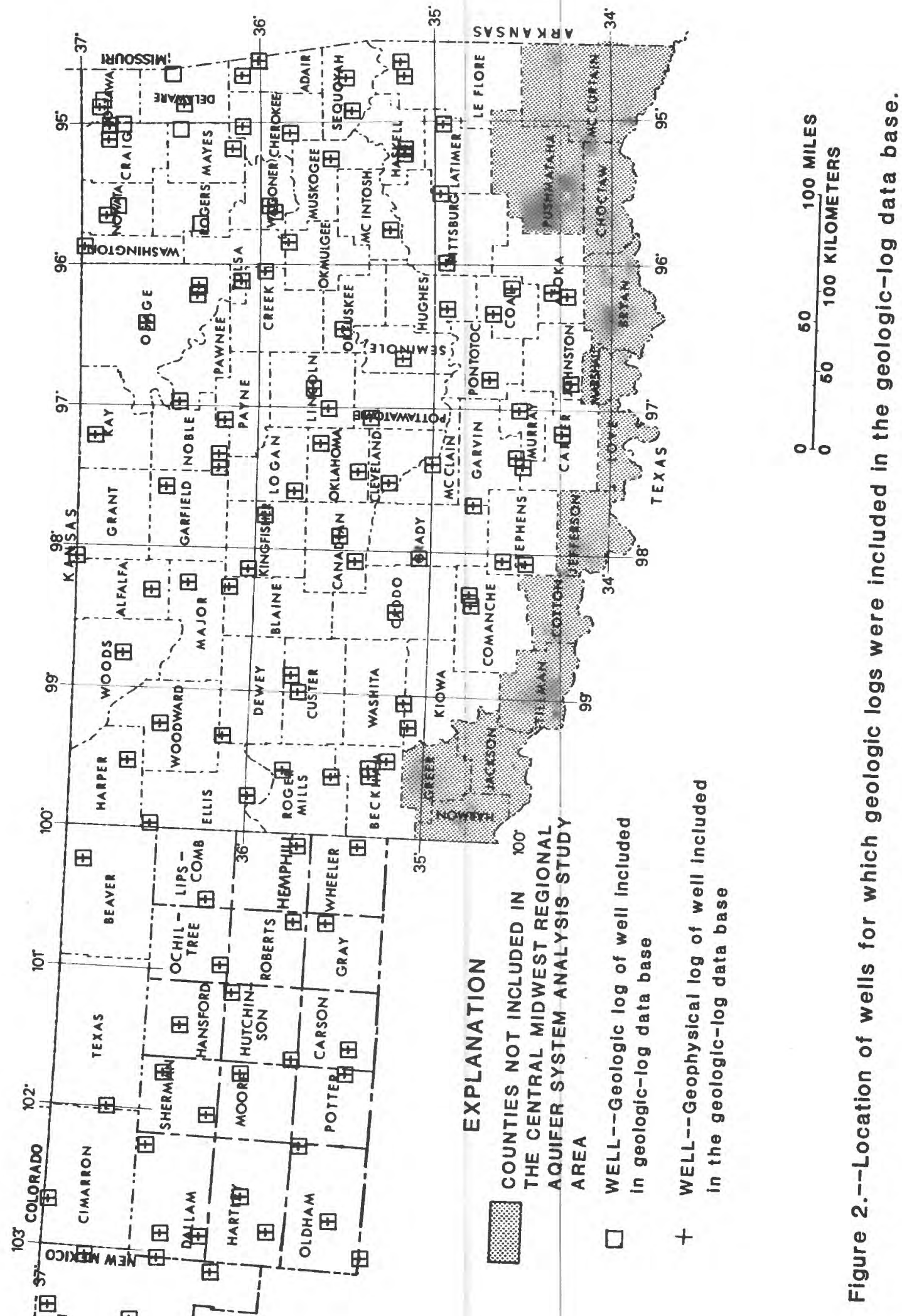




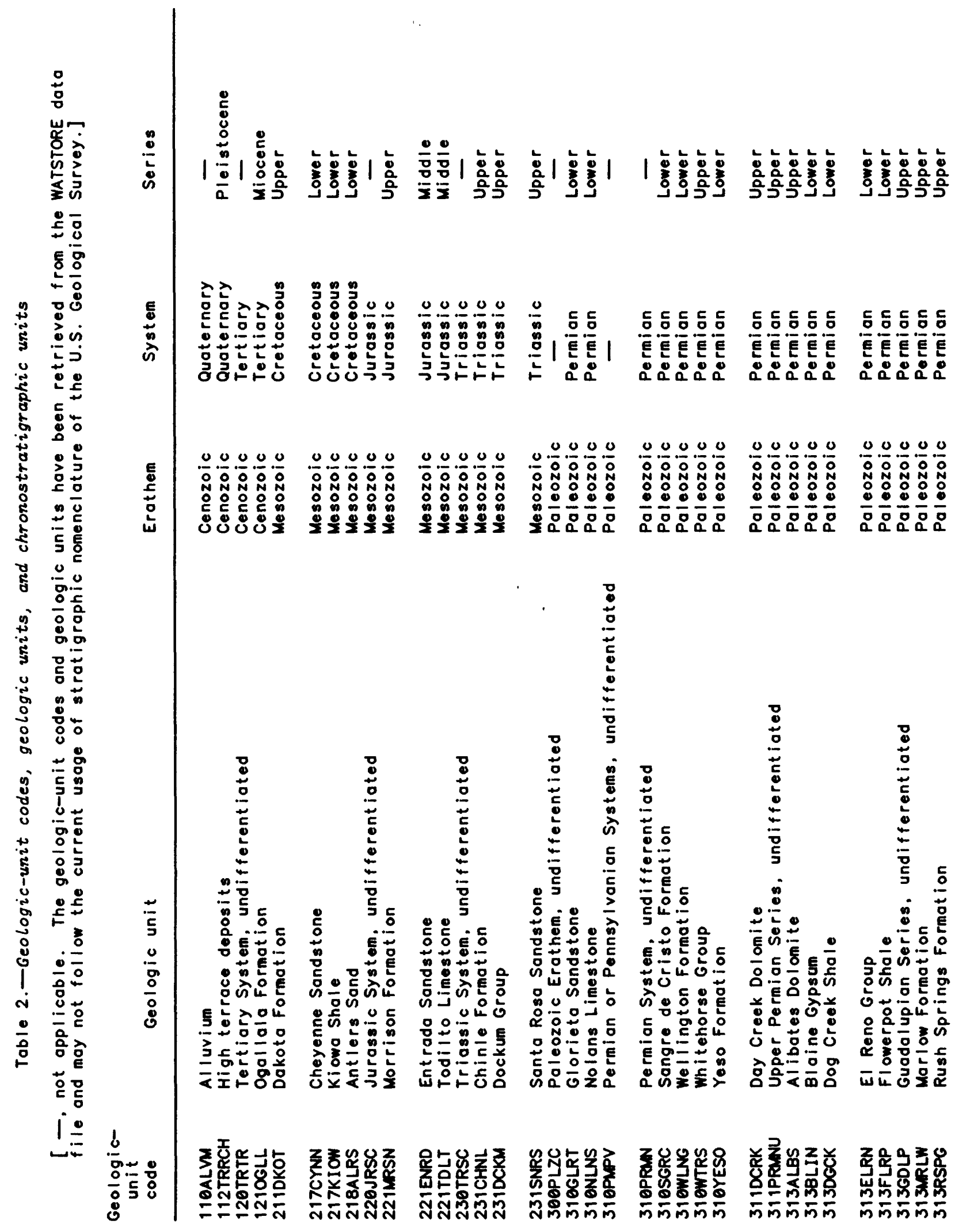




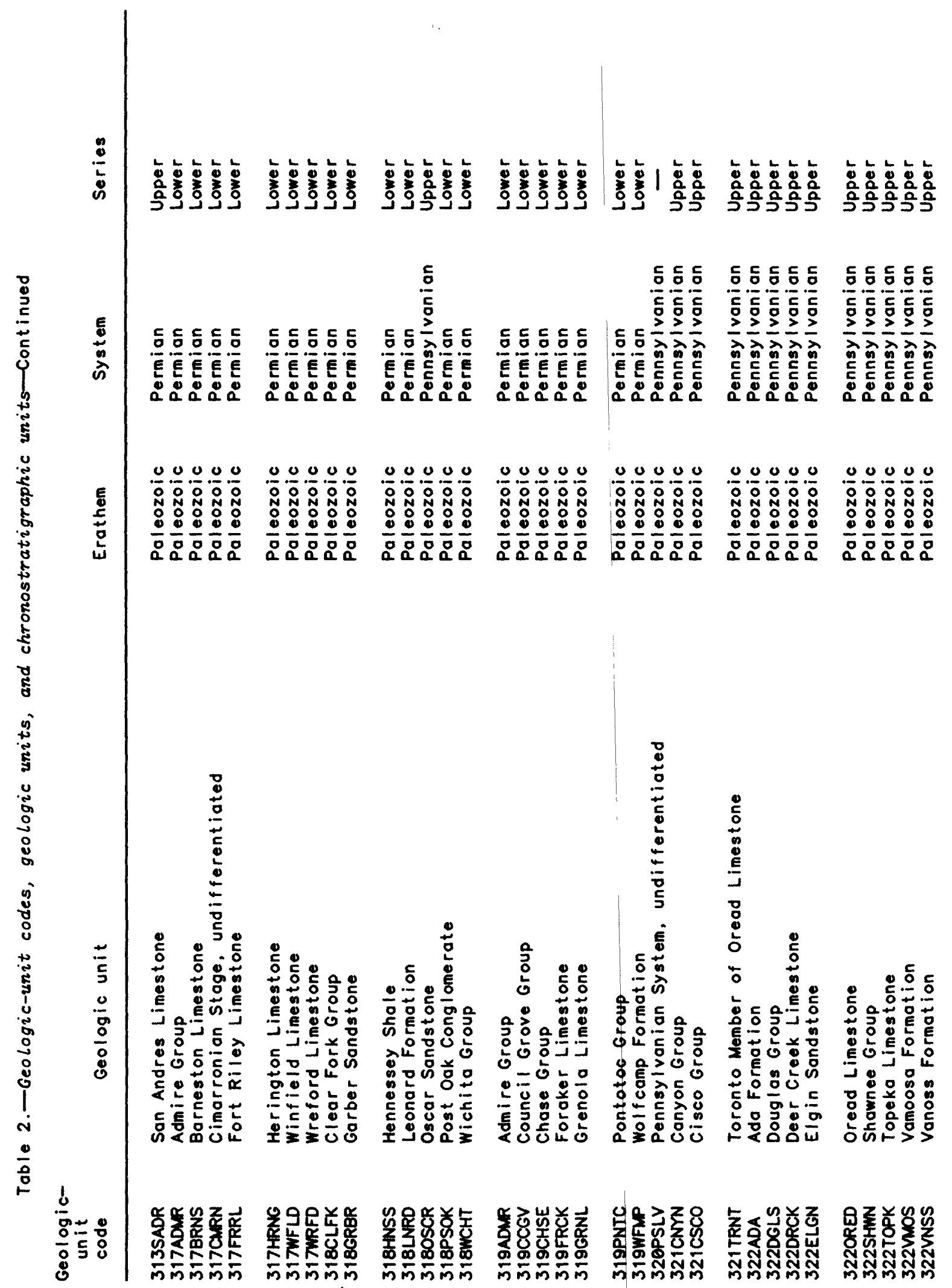




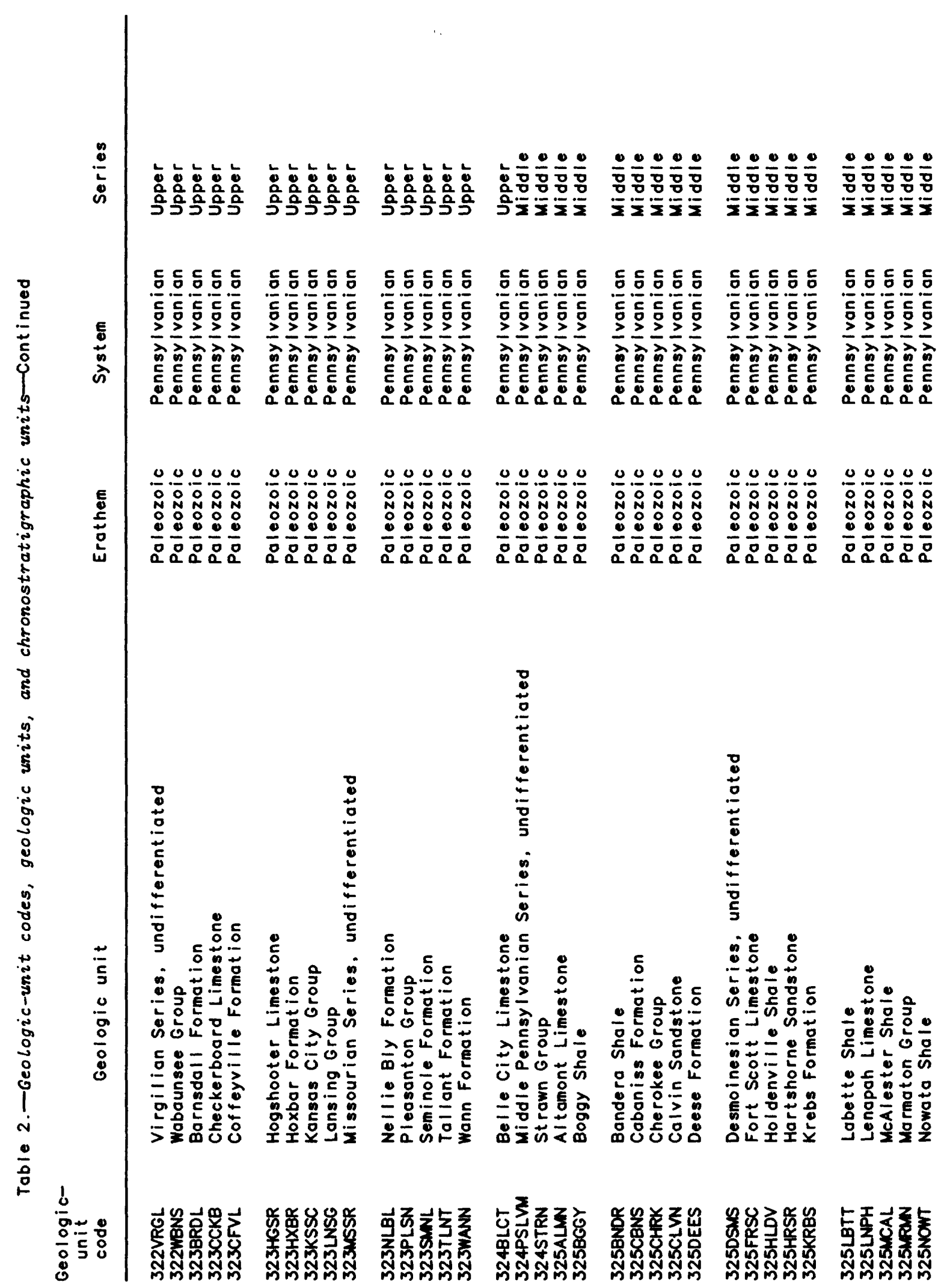




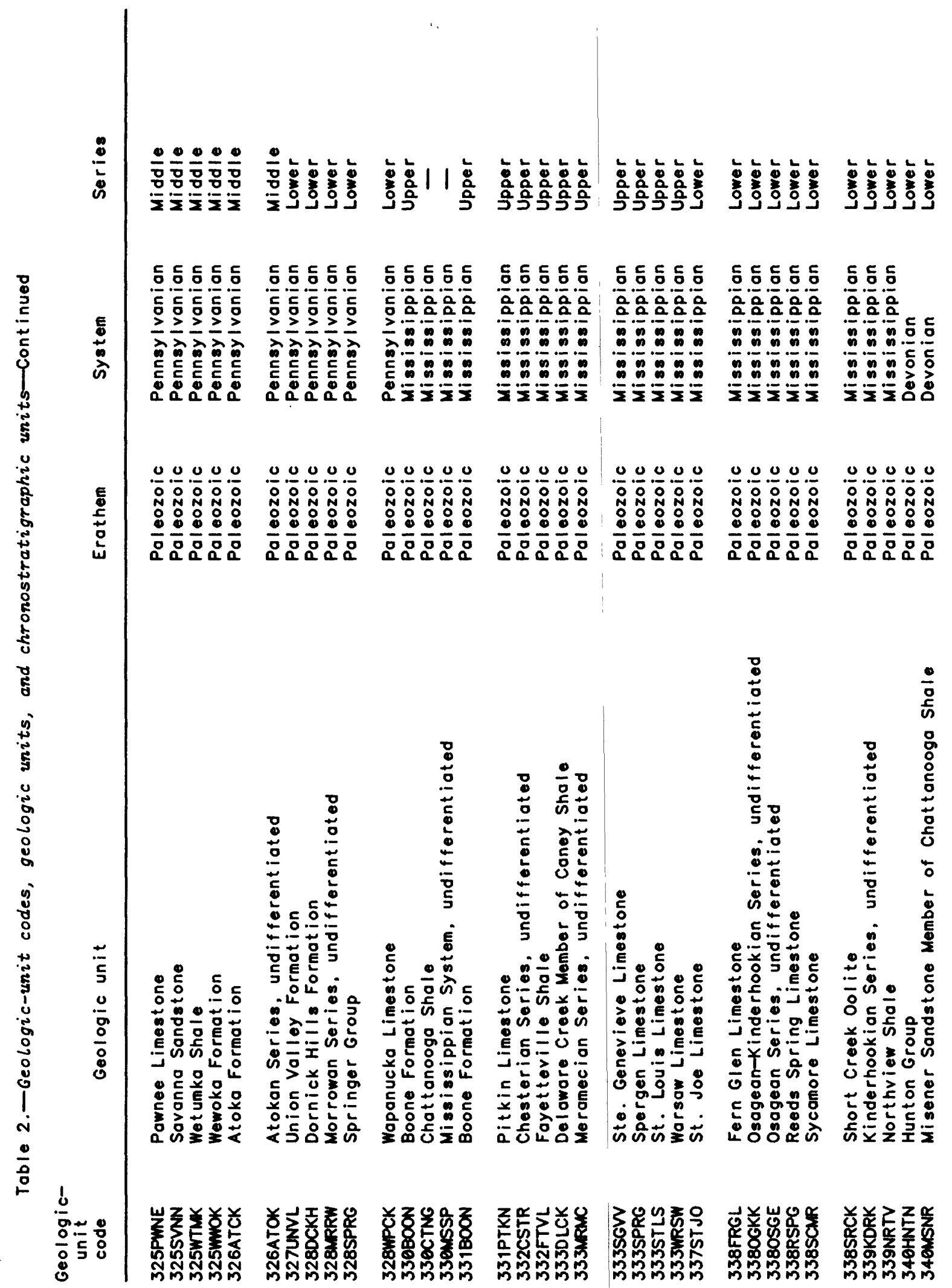




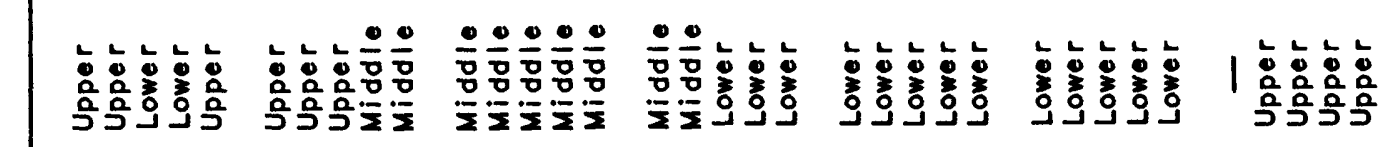

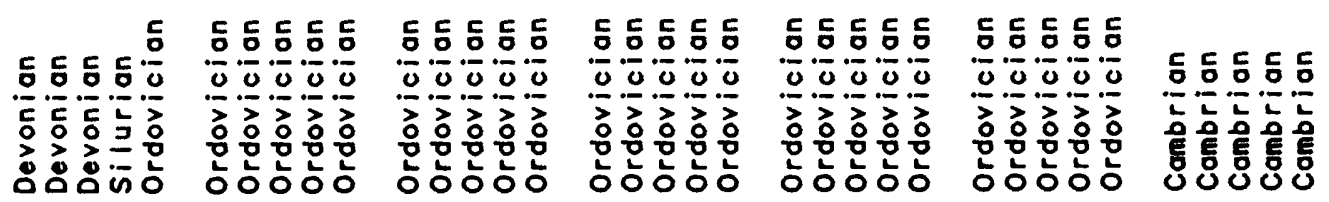

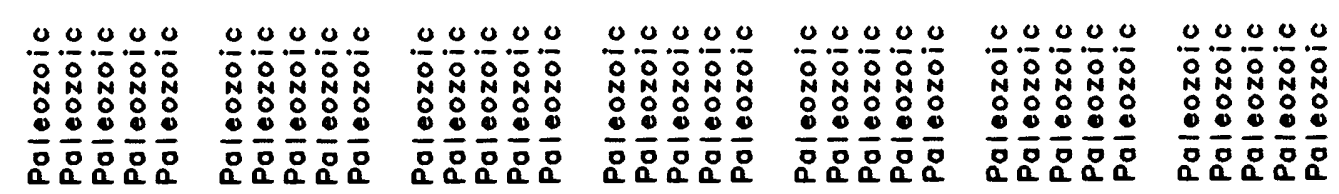

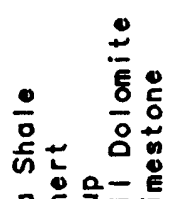

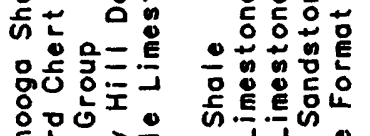

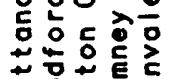

을응응

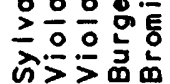

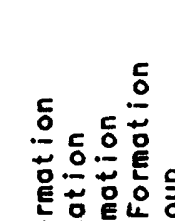

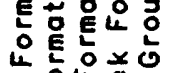

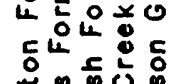

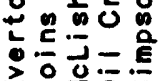

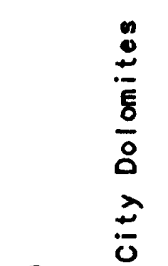

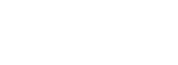

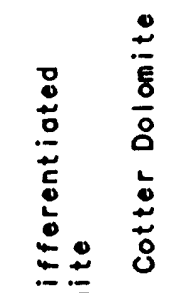

:

드응

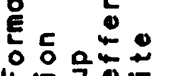

운둥흐융

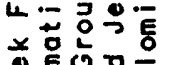

0

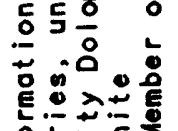

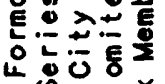

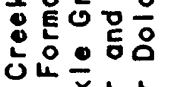

둥: 용

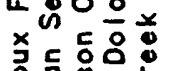

은웡

ㄴㅇㅇㅇ

응 눙

此范言

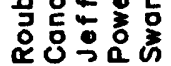

$\stackrel{8}{\frac{8}{8}}$

$\div$

웅

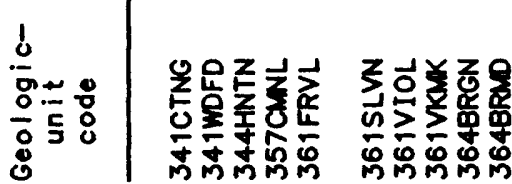
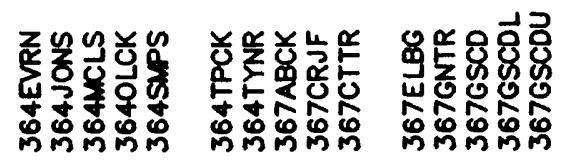

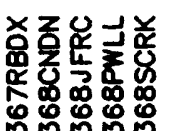

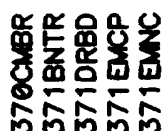




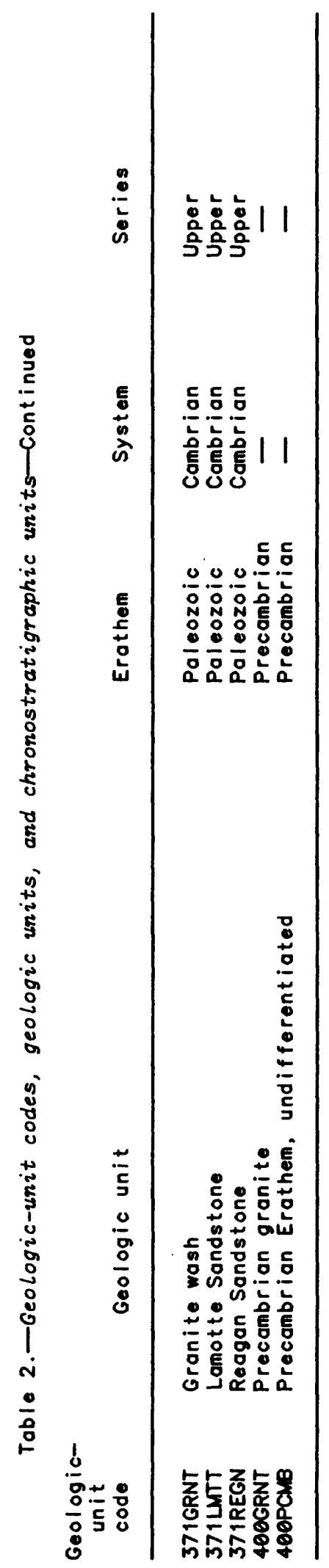




\section{SUPPLEMENTAL INFORMATION}

The geologic logs that were prepared from wells in the study area and stored in the geologic-log data base are presented in table 3. The wells are grouped by county for Oklahoma, Texas, and New Mexico. General information about each well is presented in a header, which is followed by the geologic log.

The table header contains the following information:

Latitude--latitude of the well in degrees, minutes, and seconds.

Longitude--longitude of the well in degrees, minutes, and seconds.

Owner--owner of the well at the time the log was prepared.

Local number--system for describing the tract of land where the well is located. In Oklahoma and New Mexico, this system is a version of the standard system of public land divisions (see the section "Explanation of the Site-numbering system"); in Texas, the local number is the standard system for describing public land divisions.

Use of site--an abbreviation for the principal use of the site; abbreviations are shown below:

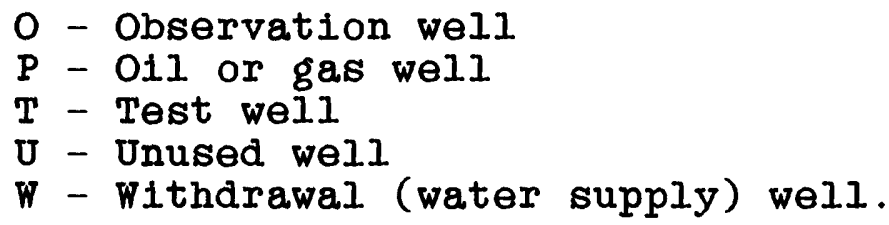

Completion date--date well completed, if known.

Altitude--altitude of land surface at the well.

Depth of well--total depth of the well.

For each well in table 3 , the header information is followed by the geologic log. Information from the geologic log includes the following:

Geologic-unit code--corresponds to the geologic unit penetrated by the wells; these codes are stored in the WATSTORE file. The term "geologic unit," for purposes of this report, is a general term for any geologic series, group, formation, or member. 
Depth to top--the depth in feet, from the land surface to the top of the geologic unit; if the geologic log for a well did not begin at the land surface, the depth to the top of the uppermost geologic unit identified in the well is the depth to the beginning of the geologic log and is not necessarily the top of the geologic unit.

Depth to bottom--depth in feet to the bottom of the geologic unit; generally left blank for the lowermost geologic unit, indicating that the well did not penetrate the full thickness of the geologic unit.

Lithology--an abbreviation for the dominant lithology of the interval between the top of the geologic unit and the bottom of the geologic unit; a list of abbreviations is shown below:

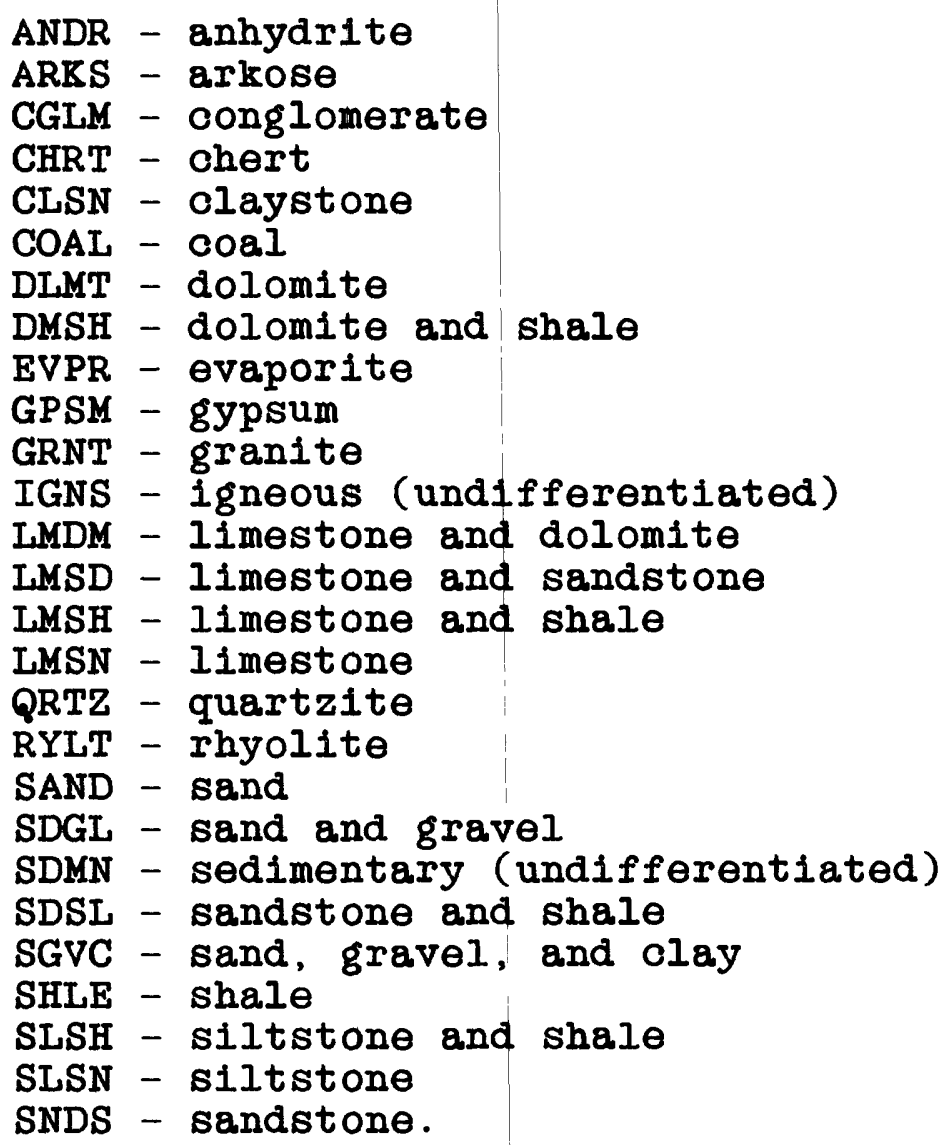

For a few welis included in table 3 , a limited number of the lithology fields are left blank. Where this ocours, the lithology could not be reliably determined for that particular stratigraphic interval. 
Some of the geologic logs in table 3 show geologic units which are repeated, indicating that more than one dominant lithology was observed within the same geologic unit. For example, in Alfalfa County, Oklahoma, for a well located at 24N-11W-12 DDA, the Wellington Formation (3lowLNG) is listed twice. For the depth interval from 1,190 to 1,535 feet below land surface, the dominant lithology in the Wellington Formation was shale. For the depth interval from 1,535 to 2,308 feet below land surface, the dominant lithology was anhydrite.

A list of geophysical logs for wells that were used as part of the process of preparing and interpreting the geologic logs is presented in table 4. The table lists the type of logs that are available and the interval that was logged. The table is grouped by State and alphabetically by county name. The header information in this table is identical to table 3. 
Table 3.--Geologic logs for wells

in the study area

Adair County, Oklahoma

Latitude: 355931

Owner: WESTVILLE OK Use of site: W

Altitude: 1,123
Longitude: 943400

Local number: 18N-26E-31 DAD 1

Completion date: 08/07/1981

Depth of well: 1,500

\begin{tabular}{lrr|c}
$\begin{array}{l}\text { Geologic- } \\
\text { unit code }\end{array}$ & $\begin{array}{r}\text { Depth to } \\
\text { top }\end{array}$ & $\begin{array}{c}\text { Depth to } \\
\text { bottom }\end{array}$ & Lithology \\
330MSSP & 10 & 248 & LMSN \\
341CTNG & 248 & 300 & SHLE \\
364SMPS & 300 & 475 & LMSN \\
368PWLL & 475 & 630 & LMSN \\
367ABCK & 630 & 1,185 & DLMT \\
367RBDX & 1,185 & 1,485 & DLMT \\
367GSCD & 1,485 & & DLMT
\end{tabular}

Adair County, Oklahoma

Lat1tude: 360507

Owner: ARTIE BROWN

Use of site:

Altitude: 1,070
Long1tude: 944022

Local number: 19N-25E-32 BDA I

Completion date:

Depth of well: 1,430

\begin{tabular}{lrr|c}
$\begin{array}{l}\text { Geologic- } \\
\text { unit code }\end{array}$ & $\begin{array}{r}\text { Depth to } \\
\text { top }\end{array}$ & $\begin{array}{c}\text { Depth to } \\
\text { bottom }\end{array}$ & Lithology \\
& 0 & 289 & IMSN \\
331BOON & 0 & 364 & SHLE \\
341CTNG & 289 & SAND \\
364BRGN & 364 & 484 & DIMT \\
368CNDN & 484 & 1,395 & GRNT
\end{tabular}


Table 3.--Geologic logs for wells in the study area--Continued

Alfalfa County, Oklahoma

Latitude: 363402

Owner: OLSON DRLG

Use of site: T

Altitude: 1,379
Long1tude: 981901

Local number: $24 \mathrm{~N}-11 \mathrm{~W}-12$ DDA 1

Completion date: 09/05/1942

Depth of well: 7,300

$\begin{array}{lrcc}\begin{array}{c}\text { Geologic- } \\ \text { untt code }\end{array} & \begin{array}{r}\text { Depth } \\ \text { top }\end{array} & \begin{array}{c}\text { Depth to } \\ \text { bottom }\end{array} & \text { Lithology } \\ \text { 310PRMN } & 40 & 1,190 & \text { SHLE } \\ \text { 31OWLNG } & 1,190 & 1,535 & \text { SHLE } \\ \text { 31OWLNG } & 1,535 & 2,308 & \text { ANDR } \\ \text { 31ONLNS } & 2,308 & 2,385 & \text { LMSH } \\ \text { 317WFLD } & 2,385 & 2,472 & \text { LMSH } \\ \text { 317BRNS } & 2,472 & 2,686 & \text { LMSH } \\ \text { 317WRFD } & 2,686 & 2,744 & \text { LMSN } \\ \text { 319CGGV } & 2,744 & 3,172 & \text { LMSH } \\ \text { 317ADMR } & 3,172 & 3,565 & \text { LMSH } \\ \text { 322WBNS } & 3,565 & 4,140 & \text { SHLE } \\ \text { 322TOPK } & 4,140 & 4,206 & \text { LMSN } \\ \text { 322SHWN } & 4,206 & 4,593 & \text { SHLE } \\ \text { 322VMOS } & 4,593 & 4,720 & \text { LMSH } \\ \text { 322DGLS } & 4,720 & 4,884 & \text { SHLE } \\ \text { 323LNSG } & 4,884 & 5,086 & \text { SNDS } \\ \text { 323KSSC } & 5,086 & 5,274 & \text { SHLE } \\ \text { 323MSSR } & 5,274 & 5,285 & \text { LMSN } \\ \text { 323MSSR } & 5,285 & 5,477 & \text { SDSL } \\ \text { 323HGSR } & 5,477 & 5,510 & \text { LMSN } \\ \text { 323MSSR } & 5,510 & 5,665 & \text { SHLE } \\ \text { 323PLSN } & 5,665 & 5,733 & \text { SHLE } \\ \text { 325MRMN } & 5,733 & 5,903 & \text { LMSN } \\ \text { 325DSMS } & 5,903 & 6,145 & \text { SHLE } \\ \text { 332CSTR } & 6,145 & 6,230 & \text { SHLE } \\ \text { 333MRMC } & 6,230 & 6,475 & \text { LMSN } \\ \text { 338OSGE } & 6,475 & 6,854 & \text { LMSN } \\ \text { 339KDRK } & 6,854 & 6,912 & \text { LMSH } \\ \text { 341WDFD } & 6,912 & 6,960 & \text { SHLE } \\ \text { 340MSNR } & 6,960 & 6,965 & \text { SNDS } \\ \text { 361SLVN } & 6,965 & 7,054 & \text { SHLE } \\ \text { 361VIOL } & 7,054 & 7,100 & \text { LMSN } \\ \text { 364BRMD } & 7,100 & & \text { LMSD } \\ & & & \end{array}$


Table 3.--Geologic logs for wells in the study area--Continued

Atoka County, Oklahoma

Latitude: 342016

Owner: LEWIS

Use of site: $P$

Altitude:

599

Longitude: 961130

Local number: 02S-11E-3I CA 1

Completion date: 03/15/1958

Depth of well: 11,833

\begin{tabular}{lrr|r}
$\begin{array}{l}\text { Geologic- } \\
\text { unit code }\end{array}$ & $\begin{array}{r}\text { Depth } \\
\text { top }\end{array}$ & $\begin{array}{r}\text { Depth to } \\
\text { bottom }\end{array}$ & Lithology \\
218ALRS & 50 & 80 & SHLE \\
326ATCK & 80 & 590 & SHLE \\
326ATCK & 590 & 670 & SNDS \\
326ATCK & 670 & 980 & SHLE \\
326ATCK & 980 & 2,440 & SHLE \\
326ATCK & 2,440 & 2,940 & SHLE \\
326ATCK & 2,940 & 3,810 & SHLE \\
326ATCK & 3,810 & 6,630 & SHLE \\
326ATCK & 6,630 & 6,700 & SDSL \\
326ATCK & 6,700 & 7,000 & SHLE \\
326ATCK & 7,000 & 7,665 & SHLE \\
328WPCK & 7,665 & 7,970 & LMSH \\
328WPCK & 7,970 & 8,400 & SHLE \\
328WPCK & 8,400 & 9,010 & SHLE \\
333DLCK & 9,010 & 9,240 & LMSN \\
338OGKK & 9,240 & 9,390 & LMSN \\
341WDFD & 9,390 & 9,660 & SHLE \\
340HNTN & 9,660 & 9,715 & LMSN \\
357CMNL & 9,715 & 9,790 & LMSN \\
361SLVN & 9,790 & 9,925 & SHLE \\
361VIOL & 9,925 & 10,200 & LMSN \\
364SMPS & 10,200 & 10,710 & LMDM \\
364SMPS & 10,710 & 10,820 & DLMT \\
364SMPS & 10,820 & 10,880 & SNDS \\
364SMPS & 10,880 & 10,980 & DMSH \\
364SMPS & 10,980 & 11,210 & LMDM \\
364SMPS & 11,210 & 11,580 & SNDS \\
367ABCK & 11,580 & & DLMT
\end{tabular}


Table 3.--Geologic logs for wells in the study area-Continued

Atoka County, Oklahoma

Latitude: 341450 Owner: CROSSLAND Use of site: P Altitude :
512
Longitude: 961304

Local number: 03S-10E-35 DD 1 Completion date: $10 / 08 / 1973$

Depth of well: 8,125

\begin{tabular}{|c|c|c|c|}
\hline $\begin{array}{l}\text { Geologic- } \\
\text { unit code }\end{array}$ & $\begin{array}{l}\text { Depth to } \\
\text { top }\end{array}$ & $\begin{array}{l}\text { Depth to } \\
\text { bottom }\end{array}$ & Lithology \\
\hline 218ALRS & 0 & 120 & SNDS \\
\hline 326ATCK & 120 & 680 & SHLE \\
\hline 326ATCK & 680 & 810 & SDSL \\
\hline 326ATCK & 810 & 1,440 & SHLE \\
\hline 326ATCK & 1,440 & 1.780 & SNDS \\
\hline 326ATCK & 1.780 & 1,990 & SHLE \\
\hline 328WPCK & 1,990 & 2,095 & LMSH \\
\hline 328WPCK & 2.095 & 3,320 & SHLE \\
\hline 333DLCK & 3,320 & 3,500 & SHLE \\
\hline 3380GKK & 3,500 & 3,720 & LMSN \\
\hline 341WDFD & 3,720 & 3,820 & SHLE \\
\hline 367ABCK & 3,820 & 3,850 & DLMT \\
\hline 367ABCK & 3,850 & 3,900 & SNDS \\
\hline $367 \mathrm{ABCK}$ & 3,900 & 5,630 & LMDM \\
\hline 367ABCK & 5,630 & 5,670 & SNDS \\
\hline $367 \mathrm{ABCK}$ & 5,670 & 6,000 & DLMT \\
\hline $367 \mathrm{ABCK}$ & 6.000 & 7,920 & DLMT \\
\hline 371REGN & 7,920 & 7,980 & SNDS \\
\hline 400РCMB & 7,980 & & GRNT \\
\hline
\end{tabular}


Table 3.--Geologic logs for wells in the study area--Continued

Beaver County, Oklahoma

Latitude: 365335

Owner: PURE OIL Use of site: T Altitude: $\quad 2,597$
Longitude: 1001507

Local number: 05N-26ECM-16 CDD 1 Completion date: $12 / 30 / 1951$

Depth of well: 9,240

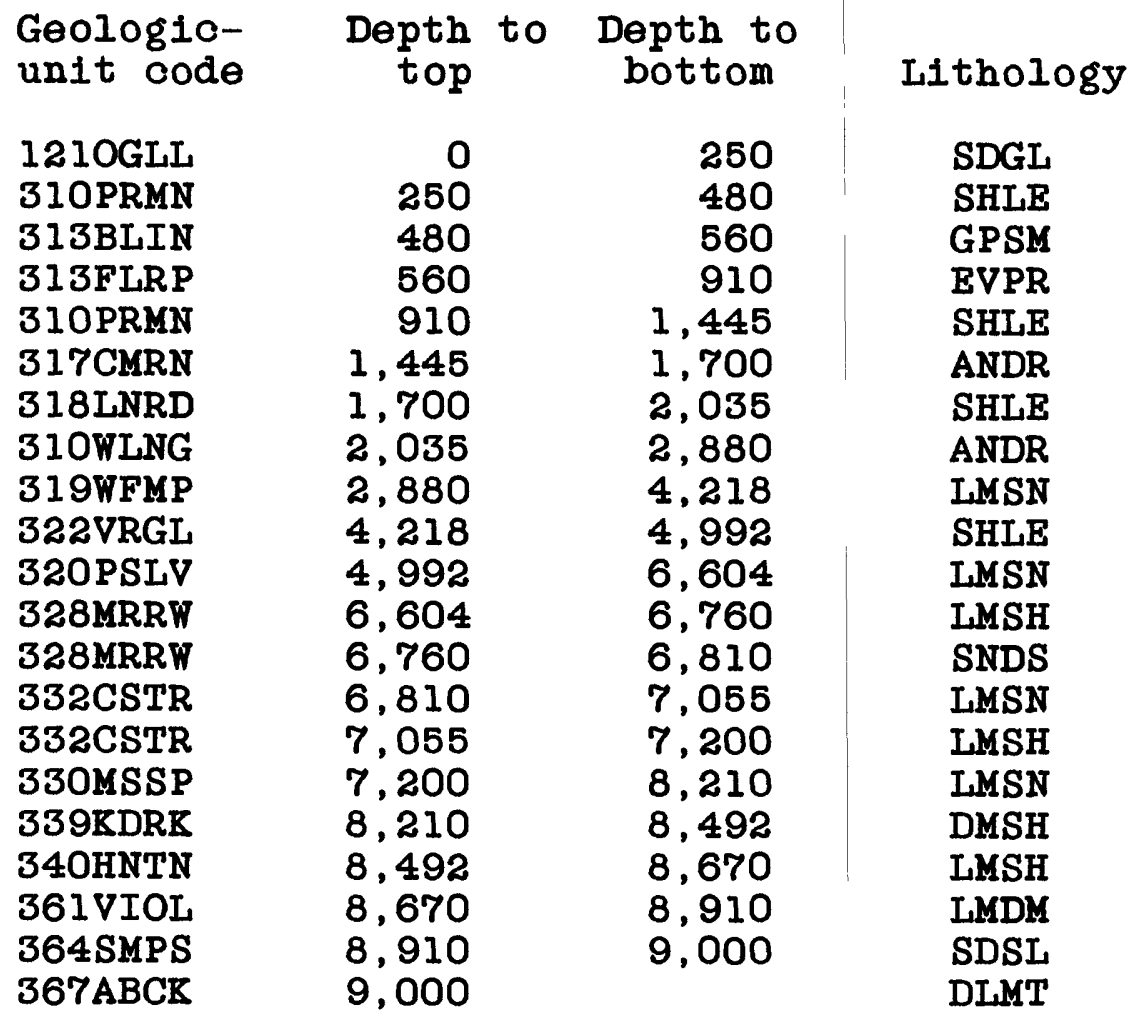

Beckham County, Oklahoma

Latitude: 351225

Owner: CARTER

Use of site: P

Altitude: $\quad 1,799$
Longitude: 992823

Local number: 09N-22W-36 DDB 1 Completion date: 05/22/1951

Depth of well: 3,100

\begin{tabular}{lrr|r}
$\begin{array}{l}\text { Geologic- } \\
\text { unit code }\end{array}$ & $\begin{array}{r}\text { Depth to } \\
\text { top }\end{array}$ & $\begin{array}{r}\text { Depth to } \\
\text { bottom }\end{array}$ & Lithology \\
31OPRMN & 259 & 380 & SNDS \\
31OPRMN & 380 & 530 & GPSM \\
31OPRMN & 530 & 1,180 & SHLE \\
31OPRMN & 1,180 & 1,420 & SHLE \\
31OPRMN & 1,420 & 2,015 & SHLE \\
31OPRMN & 2,015 & 2,095 & SHLE \\
31OWLNG & 2,095 & 2,722 & SHLE \\
31OPRMN & 2,722 & & ARES
\end{tabular}


Table 3.--Geologic logs for wells in the study area-Continued

Beckham County, Oklahoma

Latitude: 351835

Owner: LONE STAR Use of site: P Altitude: $\quad 1,953$
Longitude: 993142

Local number: 10N-22W-28 DCA 1

Completion date: 10/20/1972

Depth of well: 30,000

$\begin{array}{lrcc}\begin{array}{l}\text { Geologic- } \\ \text { unit code }\end{array} & \begin{array}{r}\text { Depth to } \\ \text { top }\end{array} & \begin{array}{r}\text { Depth to } \\ \text { bottom }\end{array} & \text { Lithology } \\ \text { 321CSCO } & 5,160 & 5,900 & \text { SHLE } \\ \text { 321CSCO } & 5,900 & 7,180 & \text { LMSH } \\ \text { 321CSCO } & 7,180 & 8,060 & \text { SHLE } \\ \text { 321CSCO } & 8,060 & 8,750 & \text { ARKS } \\ \text { 321CSCO } & 8,750 & 9,040 & \text { SHLE } \\ \text { 323HXBR } & 9,040 & 9,320 & \text { SDSL } \\ \text { 323HXBR } & 9,320 & 9,740 & \text { SDSL } \\ \text { 323HXBR } & 9,740 & 10,570 & \text { ARKS } \\ \text { 323HXBR } & 10,570 & 10,920 & \text { SDSL } \\ \text { 323HXBR } & 10,920 & 14,470 & \text { ARKS } \\ \text { 320PSLV } & 14,470 & 14,630 & \text { CGLM } \\ \text { 320PSLV } & 14,630 & 17,700 & \text { LMSH } \\ \text { 328MRRW } & 17,700 & 21,320 & \text { SHLE } \\ \text { 328MRRW } & 21,320 & 23,195 & \text { SHLE } \\ \text { 332CSTR } & 23,195 & 25,630 & \text { SHLE } \\ \text { 333MRMC } & 25,630 & 28,130 & \text { LMSH } \\ \text { 341WDFD } & 28,130 & 28,480 & \text { SHLE } \\ \text { 341WDFD } & 28,480 & 28,610 & \text { LMSN } \\ \text { 34OHNTN } & 28,610 & 28,740 & \text { LMSN } \\ \text { 34OHNTN } & 28,740 & 29,565 & \text { LMSN } \\ \text { 357CMNL } & 29,565 & 29,770 & \text { LMSN } \\ \text { 361SLVN } & 29,770 & 29,965 & \text { SHLE } \\ \text { 361VIOL } & 29,965 & & \text { LMSN }\end{array}$


Table 3.--Geologic logs for wells in the study area--Continued

\section{Beckham County, Oklahoma}

Latitude: 351816

Owner: CARTER Use of site: P

Altitude: 1,828
Longitude: 993453

Local number: $10 \mathrm{~N}-23 \mathrm{~W}-36$ ABA 1 Completion date: $09 / 28 / 1950$

Depth of well: 10,654

\begin{tabular}{lrc|c}
$\begin{array}{r}\text { Geologic- } \\
\text { unit code }\end{array}$ & $\begin{array}{r}\text { Depth } \\
\text { top }\end{array}$ & $\begin{array}{c}\text { Depth to } \\
\text { bottom }\end{array}$ & Lithology \\
313ELRN & 0 & 1,010 & SNDS \\
313BLIN & 1,010 & 1,430 & ANDR \\
313ELRN & 1,430 & 2,500 & SHLE \\
318WCHT & 2,500 & 3,020 & SHLE \\
310PRMN & 3,020 & 4,270 & SHLE \\
321CSCO & 4,270 & 4,585 & DMSH \\
321CSCO & 4,585 & 4,835 & LMSH \\
321CSCO & 4,835 & 6,685 & SHLE \\
321CSCO & 6,685 & 6,950 & LMSN \\
321CSCO & 6,950 & 7,380 & SHLE \\
321CSCO & 7,380 & 8,280 & ARKS \\
321CSCO & 8,280 & 8,560 & LMSH \\
321CSCO & 8,560 & 9,060 & ARKS \\
323HXBR & 9,060 & 9,665 & ARKS \\
323HXBR & 9,665 & & ARKS
\end{tabular}


Table 3.--Geologic logs for wells in the study area--Continued

\section{Blaine County, Oklahoma}

Latitude: 360746 Owner: SUPERIOR Use of site: P Altitude: 1,200
Longitude: 981658

Local number: 19N-10W-17 AAA 1 Completion date: 01/01/1945

Depth of well: 11,008

\begin{tabular}{|c|c|c|c|}
\hline $\begin{array}{l}\text { Geologic- } \\
\text { unit code }\end{array}$ & $\begin{array}{l}\text { Depth to } \\
\text { top }\end{array}$ & $\begin{array}{l}\text { Depth to } \\
\text { bottom }\end{array}$ & Iithology \\
\hline 313FLRP & 0 & 70 & SHLE \\
\hline $318 \mathrm{HNSS}$ & 70 & 1,310 & SHLE \\
\hline 310WLNG & 1,310 & 1,667 & SHLE \\
\hline 310WLNG & 1,667 & 2,482 & ANDR \\
\hline 310NLNS & 2,482 & 2,546 & DMSH \\
\hline 317WFLD & 2,546 & 2,614 & LMSN \\
\hline 319CHSE & 2,614 & 2,640 & SHLE \\
\hline 317BRNS & 2,640 & 2,790 & LMSH \\
\hline $319 \mathrm{CHSE}$ & 2,790 & 2,807 & SHLE \\
\hline 317WRFD & 2,807 & 2,896 & LMSN \\
\hline 319CCGV & 2,896 & 2,982 & SHLE \\
\hline 319CCGV & 2,982 & 3,074 & LMSH \\
\hline 319CCGV & 3,074 & 3,166 & SHLE \\
\hline 319GRNL & 3,166 & 3,220 & LMSH \\
\hline 319CCGV & 3,220 & 3,327 & SHLE \\
\hline $319 \mathrm{CCGV}$ & 3,327 & 3,428 & LMSN \\
\hline 319ADMR & 3,428 & 4,028 & LMSH \\
\hline 322WBNS & 4,028 & 4,100 & LMSN \\
\hline 322WBNS & 4,100 & 4,208 & LMSH \\
\hline 322WBNS & 4,208 & 4,417 & LMSN \\
\hline 322WBNS & 4,417 & 4,570 & SHLE \\
\hline 322TOPK & 4,570 & 4,624 & LMSN \\
\hline 322SHWN & 4,624 & 4,752 & SHLE \\
\hline 322SHWN & 4,752 & 4,832 & LMSN \\
\hline 322SHWN & 4,832 & 5,264 & SHLE \\
\hline 322ORED & 5,264 & 5,395 & LMSN \\
\hline 322DGLS & 5,395 & 5,620 & SHLE \\
\hline 323LNSG & 5,620 & 5,737 & SNDS \\
\hline 323KSSC & 5,737 & 6,158 & SHLE \\
\hline 323MSSR & 6,158 & 6,267 & SNDS \\
\hline 323MSSR & 6,267 & 6,484 & SHLE \\
\hline 323HGSR & 6,484 & 6,487 & LMSN \\
\hline 323MSSR & 6,487 & 6,713 & SHLE \\
\hline 323 PLSN & 6,713 & 6,820 & SHLE \\
\hline 325MRMN & 6.820 & 7,069 & LMSN \\
\hline 325CBNS & 7,069 & 7,360 & SHLE \\
\hline 332CSTR & 7,360 & 7,460 & LMSH \\
\hline 332CSTR & 7,460 & 7,628 & SHLE \\
\hline 332CSTR & 7,628 & 7,791 & LMSH \\
\hline
\end{tabular}


Table 3.--Geologic logs for wells in the study area--Continued

Blaine County, Oklahoma--Continued

Latitude: 360746

Owner: SUPERIOR

Use of site: P

Altitude: 1,200
Long1tude: 981658

Local number: 19N-10W-17 AAA 1

Completion date: 01/01/1945

Depth of well: 11,008
Geologic- Depth to Depth to unit code

top

bottom
7,791

7,926

8,525

8,533

8,580

8,616

8,870

8,978

9,040

9,289

9,480

9,721
7,926

8,525

8,533

8,580

8,616

8,870

8,978

9,040

9,289

9,480

9,721

\section{IIthology}

SHLE

LMSN

SHLE

SHLE

DLMT

LMSH

SHLE

IMSN

IMSH

SNDS

SDSL

DLMT 
Table 3.--Geologic logs for wells in the study area--Continued

Caddo County, Oklahoma

Latitude: 351119

Owner: SUPERIOR

Use of site: P

Altitude: 1,440
Longitude: 982556

Local number: 08N-12W-11 ABB 1

Completion date: 01/01/1947

Depth of well: 17,823

\begin{tabular}{|c|c|c|c|}
\hline $\begin{array}{l}\text { Geologic- } \\
\text { untt code }\end{array}$ & $\begin{array}{c}\text { Depth to } \\
\text { top }\end{array}$ & $\begin{array}{l}\text { Depth to } \\
\text { bottom }\end{array}$ & Lithology \\
\hline $313 \mathrm{RSPG}$ & 0 & 250 & SNDS \\
\hline 313MRLW & 250 & 390 & SNDS \\
\hline 313 ELRN & 390 & 617 & SHLE \\
\hline 313FLRP & 617 & 780 & SHLE \\
\hline 310GLRT & 780 & 1,076 & SDSL \\
\hline $318 \mathrm{HNSS}$ & 1,076 & 2,225 & SHLE \\
\hline $318 \mathrm{GRBR}$ & 2,225 & 2,850 & SHLE \\
\hline 310WLNG & 2,850 & 3,480 & SHLE \\
\hline 310WLNG & 3,480 & 3,894 & ANDR \\
\hline 319CHSE & 3,894 & 4,000 & DMSH \\
\hline 319CHSE & 4,000 & 4,210 & SHLE \\
\hline 319CHSE & 4,210 & 4,230 & LMSN \\
\hline $319 \mathrm{CHSE}$ & 4,230 & 4,254 & DMSH \\
\hline 317 WRFD & 4,254 & 4,350 & LMSH \\
\hline $319 \mathrm{CCGV}$ & 4,350 & 4,431 & SDSL \\
\hline $319 \mathrm{CCGV}$ & 4,431 & 4,616 & LMSH \\
\hline $319 \mathrm{CCGV}$ & 4,616 & 4,853 & SDSL \\
\hline $319 \mathrm{ADMR}$ & 4,853 & 5,805 & SDSL \\
\hline 322WBNS & 5,805 & 6,880 & SDSL \\
\hline 322 SHWN & 6,880 & 7,850 & SLSH \\
\hline 322 SHWN & 7,850 & 8,207 & SHLE \\
\hline 322 SHWN & 8,207 & 8,390 & SNDS \\
\hline 322DGLS & 8,390 & 8,465 & SLSN \\
\hline 322DGLS & 8,465 & 8,524 & SHLE \\
\hline 323LNSG & 8,524 & 8,794 & SNDS \\
\hline $323 \mathrm{KSSC}$ & 8,794 & 9,120 & SLSH \\
\hline $323 \mathrm{KSSC}$ & 9,120 & 9,360 & SHLE \\
\hline 323MSSR & 9,360 & 9,568 & SHLE \\
\hline 323MSSR & 9,568 & 9,670 & SDSL \\
\hline $323 \mathrm{MSSR}$ & 9,670 & 9,750 & SHLE \\
\hline $323 \mathrm{MSSR}$ & 9,750 & 9,850 & SNDS \\
\hline $323 \mathrm{MSSR}$ & 9,850 & 9,920 & SHLE \\
\hline 323HGSR & 9,920 & 9,994 & SLSN \\
\hline $323 \mathrm{MSSR}$ & 9,994 & 10,420 & SDSL \\
\hline $323 \mathrm{MSSR}$ & 10,420 & 10,640 & SHLE \\
\hline 323 PLSN & 10,640 & 10,765 & SHLE \\
\hline 325DEES & 10,765 & 11,075 & SHLE \\
\hline 325DEES & 11,075 & 11,216 & SNDS \\
\hline 325DEES & 11,216 & 11,310 & SLSN \\
\hline
\end{tabular}


Table 3.--Geologic logs for wells in the study area-Continued

Caddo County, Oklahoma--Continued

Latitude: 351119

Owner: SUPERIOR

Use of site: $P$

Altitude: 1,440
Longitude: 982556

Local number: 08N-12W-11 ABB 1 Completion date: 01/01/1947

Depth of well: 17,823

\begin{tabular}{lrc|c}
$\begin{array}{c}\text { Geologic- } \\
\text { unit code }\end{array}$ & $\begin{array}{r}\text { Depth } \\
\text { top }\end{array}$ & $\begin{array}{c}\text { Depth to } \\
\text { bottom }\end{array}$ & Lithology \\
325DEES & 11,310 & 12,055 & SHLE \\
325DEES & 12,055 & 12,468 & SLSH \\
325DEES & 12,468 & 12,687 & SHLE \\
325DEES & 12,687 & 12,792 & SLSN \\
325DEES & 12,792 & 13,087 & SHLE \\
326ATCK & 13,087 & 13,310 & SHLE \\
326ATCK & 13,310 & 13,468 & SLSH \\
326ATCK & 13,468 & 14,360 & SHLE \\
326ATCK & 14,360 & 15,510 & SHLE \\
328MRRW & 15,510 & 15,875 & SHLE \\
328MRRW & 15,875 & 16,020 & SLSN \\
328MRRW & 16,020 & 16,257 & SDSL \\
328SPRG & 16,257 & 16,412 & SNDS \\
328SPRG & 16,412 & 16,606 & SHLE \\
328SPRG & 16,606 & 16,722 & SNDS \\
328SPRG & 16,722 & 16,925 & SHLE \\
328SPRG & 16,925 & 17,065 & SNDS \\
328SPRG & 17,065 & 17,566 & SHLE \\
328SPRG & 17,566 & 17,700 & SNDS \\
328SPRG & 17,700 & & SHLE
\end{tabular}


Tuble 3.--Geologic logs for wells in the study area-Continued

Canadian County, Oklahoma

Latitude: 352521

Owner: PETREE

Use of site: $P$

Altitude: 1,310
Longitude: 980450

Local number: $11 \mathrm{~N}-08 \mathrm{~W}-18$ DD 1

Completion date:

Depth of well: 11,177

$\begin{array}{lrrr}\begin{array}{c}\text { Geologic- } \\ \text { unit code }\end{array} & \begin{array}{r}\text { Depth } \\ \text { top }\end{array} & \begin{array}{r}\text { Depth to } \\ \text { bottom }\end{array} & \text { Lithology } \\ \text { 110ALVM } & 0 & 30 & \text { SGVC } \\ \text { 313ELRN } & 30 & 670 & \text { SDSL } \\ \text { 318HNSS } & 670 & 1,331 & \text { SHLE } \\ \text { 318HNSS } & 1,331 & 1,555 & \text { SDSL } \\ \text { 318GRBR } & 1,55 & 2,382 & \text { SDSL } \\ \text { 310WLNG } & 2,382 & 2,687 & \text { SHLE } \\ \text { 310WLNG } & 2,687 & 3,290 & \text { SHLE } \\ \text { 319CHSE } & 3,290 & 3,306 & \text { LMSN } \\ \text { 319CHSE } & 3,306 & 3,478 & \text { SHLE } \\ \text { 317BRNS } & 3,478 & 3,614 & \text { LMSN } \\ \text { 319CHSE } & 3,614 & 3,649 & \text { SHLE } \\ \text { 317WRFD } & 3,649 & 3,733 & \text { SHLE } \\ \text { 319CCGV } & 3,733 & 3,863 & \text { SHLE } \\ \text { 319CGGV } & 3,863 & 4,035 & \text { SHLE } \\ \text { 319CGGV } & 4,035 & 4,240 & \text { SDSL } \\ \text { 319ADMR } & 4,240 & 5,190 & \text { SDSL } \\ \text { 322WBNS } & 5,190 & 5,339 & \text { SHLE } \\ \text { 322WBNS } & 5,339 & 5,967 & \text { SHLE } \\ \text { 322SHWN } & 5,967 & 7,498 & \text { SDSL } \\ \text { 322DGLS } & 7,498 & 7,844 & \text { SHLE } \\ \text { 323LNSG } & 7,844 & 8,063 & \text { SNDS } \\ \text { 323KSSC } & 8,063 & 8,507 & \text { SDSL } \\ \text { 323MSSR } & 8,507 & 8,826 & \text { SHLE } \\ \text { 323HGSR } & 8,826 & 8,872 & \text { SHLE } \\ \text { 323MSSR } & 8,872 & 9,008 & \text { SDSL } \\ \text { 323MSSR } & 9,008 & 9,240 & \text { SHLE } \\ \text { 323PLSN } & 9,240 & 9,370 & \text { SHLE } \\ \text { 325MRMN } & 9,370 & 9,820 & \text { SHLE } \\ \text { 325MRMN } & 9,820 & 9,900 & \text { LMSN } \\ \text { 325MRMN } & 9,900 & 10,000 & \text { SHLE } \\ \text { 325MRMN } & 10,000 & 10,076 & \text { LMSN } \\ \text { 325CBNS } & 10,076 & 10,562 & \text { SHLE } \\ \text { 326ATCK } & 10,562 & 10,775 & \text { SHLE } \\ \text { 332CSTR } & 10,775 & & \text { LMSN }\end{array}$


Table 3.--Geologic logs for wells in the study area--Continued

Canadian County, Oklahoma

Latitude: 353053 Owner : HUCHTEMAN Use of site: P Altitude: 1,344
Longitude: 975418

Local number: 12N-07W-14 DAB 1 Completion date:

Depth of well: 11,700

\begin{tabular}{|c|c|c|c|}
\hline $\begin{array}{l}\text { Geologic- } \\
\text { unit code }\end{array}$ & $\underset{\text { top }}{\text { Depth to }}$ & $\begin{array}{l}\text { Depth to } \\
\text { bottom }\end{array}$ & Lithology \\
\hline 313GDLP & 0 & 30 & SHLE \\
\hline 31OPRMN & 30 & 1,150 & SHLE \\
\hline 310PRMN & 1,150 & 1,530 & SDSL \\
\hline 310PRMN & 1,530 & 2,580 & SHLE \\
\hline 310PRMN & 2,580 & 2,750 & SHLE \\
\hline 31OPRMN & 2,750 & 4,450 & SHLE \\
\hline 322WBNS & 4,450 & 4,990 & SHLE \\
\hline 322VRGL & 4,990 & 6.080 & SDSL \\
\hline 323MSSR & 6,080 & 6,590 & SHLE \\
\hline 323MSSR & 6,590 & 6,700 & IMSN \\
\hline 323MSSR & 6,700 & 7,920 & SHLE \\
\hline 325MRMN & 7,920 & 8,314 & SHLE \\
\hline 325FRSC & 8,314 & 8,430 & IMSN \\
\hline 325CHRK & 8,430 & 8,820 & SHLE \\
\hline 332CSTR & 8,820 & 8,906 & SHLE \\
\hline 330MSSP & 8,906 & 8,950 & DLMT \\
\hline 330MSSP & 8,950 & 9,080 & SHLE \\
\hline 330MSSP & 9,080 & 9,120 & LMSN \\
\hline 33OMSSP & 9,120 & 9,220 & SHLE \\
\hline 330MSSP & 9,220 & 9,320 & IMSN \\
\hline 341WDFD & 9,320 & 9,429 & SHLE \\
\hline 340MSNR & 9,429 & 9,430 & SNDS \\
\hline 340HNTN & 9,430 & 9,630 & LMDM \\
\hline 357CMNL & 9,630 & 9,725 & LMSN \\
\hline 36ISLVN & 9,725 & 9,835 & SHLE \\
\hline 361FRVL & 9,835 & 9,850 & IMSN \\
\hline 361 VIOL & 9,850 & 10,150 & IMSN \\
\hline 364SMPS & 10,150 & 10,284 & LMDM \\
\hline 364BRMD & 10,284 & 10,615 & SNDS \\
\hline 364MCLS & 10,615 & 10,905 & SNDS \\
\hline 364OLCK & 10,905 & 11,050 & SHLE \\
\hline 367ABCK & 11,050 & & DLMT \\
\hline
\end{tabular}


Table 3.--Geologic logs for wells in the study area--Continued

Carter County, Oklahoma

Latitude: 342921

Owner: MEEKS

Use of site: $P$

Altitude :
938
Longltude: 972402

Local number: 01S-02W-10 BBD 1

Completion date: $03 / 22 / 1966$

Depth of well: 3,018

$\begin{array}{lrrc}\begin{array}{l}\text { Geologic- } \\ \text { unit code }\end{array} & \begin{array}{r}\text { Depth to } \\ \text { top }\end{array} & \begin{array}{r}\text { Depth to } \\ \text { bottom }\end{array} & \text { Lithology } \\ \text { 319PNTC } & 340 & 410 & \text { CGLM } \\ \text { 3640LCK } & 410 & 800 & \text { SHLE } \\ \text { 364OLCK } & 800 & 1,000 & \text { LMSH } \\ \text { 367ABCK } & 1,000 & & \text { LMDM }\end{array}$

Carter County, Oklahoma

Latitude: 341618

Owner: WHITE EAGLE

Use of site: $P$

Altitude: $\quad 763$
Long1tude: 971010

Local number: 03S-01E-26 BAD 1

Completion date: 10/31/1977

Depth of well: 16,950

$\begin{array}{lccc}\begin{array}{c}\text { Geologic- } \\ \text { un1t code }\end{array} & \begin{array}{r}\text { Depth to } \\ \text { top }\end{array} & \begin{array}{c}\text { Depth to } \\ \text { bottom }\end{array} & \text { Lithology } \\ \text { 361VIOL } & 4,090 & 4,900 & \text { LMSN } \\ \text { 364BRMD } & 4,900 & 5,300 & \text { LMSH } \\ \text { 364TPCK } & 5,300 & 5,850 & \text { LMSH } \\ \text { 364OLCK } & 5,850 & 6,520 & \text { SHLE } \\ \text { 364JONS } & 6,520 & 6,840 & \text { LMSN } \\ \text { 367ABCK } & 6,840 & & \text { LMSN }\end{array}$


Table 3.--Geologic logs for wells in the study area-Continued

Cherokee County, Oklahoma

Latitude: 354856

Owner: PINE

Use of site: T

Altitude: $\quad 690$
Longitude: 950444

Local number: $16 \mathrm{~N}-21 \mathrm{E}-33 \mathrm{DCA} 1$

Completion date: $01 / 01 / 1962$

Depth of well: 2,093

\begin{tabular}{lrr|c}
$\begin{array}{l}\text { Geologic- } \\
\text { unit code }\end{array}$ & $\begin{array}{r}\text { Depth to } \\
\text { top }\end{array}$ & $\begin{array}{c}\text { Depth to } \\
\text { bottom }\end{array}$ & Lithology \\
341CTNG & 62 & 113 & SHLE \\
364SMPS & 113 & 379 & SNDS \\
368PWLI & 379 & 660 & DLMT \\
367CTTR & 660 & 1,104 & DLMT \\
368JFRC & 1,104 & 1,298 & DLMT \\
367RBDX & 1,298 & 1,515 & DLMT \\
367GSCDU & 1,515 & 1,592 & DLMT \\
367GSCDL & 1,592 & 1,757 & DLMT \\
367GNTR & 1,757 & 1,820 & SNDS \\
371EMCP & 1,820 & 1,925 & DLMT \\
371BNTR & 1,925 & 2,000 & DLMT \\
371LMTT & 2,000 & 2,078 & SNDS \\
400PCMB & 2,078 & & GRNT
\end{tabular}

Cherokee County, Oklahoma

Latitude: 360502

Owner: M F OIL CO Use of site: $T$ Altitude:
800
Longitude: 950152

Local number: 19N-21E-35 AD 1

Completion date: 01/01/1961

Depth of well: 1,935

\begin{tabular}{lrr|c}
$\begin{array}{l}\text { Geologic- } \\
\text { unit code }\end{array}$ & $\begin{array}{r}\text { Depth to } \\
\text { top }\end{array}$ & $\begin{array}{c}\text { Depth to } \\
\text { bottom }\end{array}$ & Lithology \\
367CTTR & 460 & 807 & DLMT \\
368JFRC & 807 & 985 & DLMT \\
367RBDX & 985 & 1,180 & DLMT \\
367GSCD & 1,180 & 1,525 & DLMT \\
371EMNC & 1,525 & 1,660 & DLMT \\
371BNTR & 1,660 & 1,730 & DLMT \\
371LMTT & 1,730 & 1,750 & SNDS \\
371GRNT & 1,750 & 1,800 & ARKS
\end{tabular}


Table 3.--Geologic logs for wells in the study area--Continued

Cimarron County, Oklahoma

Latitude: 365818

Owner: SUN CO.

Use of site: T

Altitude: 4,305
Long1tude: 1024134

Local number: 06N-03E-24 CB 1

Completion date: $06 / 24 / 1954$

Depth of well: 5,904

$\begin{array}{lrrc}\begin{array}{l}\text { Geologic- } \\ \text { unit code }\end{array} & \begin{array}{r}\text { Depth } \\ \text { top }\end{array} & \begin{array}{r}\text { Depth to } \\ \text { bottom }\end{array} & \text { Lithology } \\ \text { 211DKOT } & 0 & 96 & \text { SNDS } \\ \text { 217KIOW } & 96 & 142 & \text { SHLE } \\ 217 \text { SYNN } & 142 & 220 & \text { SNDS } \\ \text { 221MRSN } & 220 & 492 & \text { SHLE } \\ \text { 221ENRD } & 492 & 520 & \text { SNDS } \\ \text { 231DCKM } & 520 & 875 & \text { SDSL } \\ \text { 311DCRK } & 875 & 1,008 & \text { ANDR } \\ \text { 311PRMN } & 1,008 & 1,263 & \text { SHLE } \\ \text { 313BLIN } & 1,263 & 1,470 & \text { ANDR } \\ \text { 310GLRT } & 1,470 & 1,570 & \text { SNDS } \\ \text { 310PRMN } & 1,570 & 2,960 & \text { SLSH } \\ \text { 319WFMP } & 2,960 & 3,468 & \text { SHLE } \\ \text { 320PSLV } & 3,468 & 3,890 & \text { LMSH } \\ \text { 328MRRW } & 3,890 & 4,470 & \text { SDSL } \\ \text { 328MRRW } & 4,470 & 4,850 & \text { SHLE } \\ \text { 333SGVV } & 4,850 & 4,910 & \text { LMSN } \\ \text { 333STLS } & 4,910 & 5,060 & \text { LMSN } \\ \text { 333SPRG } & 5,060 & 5,140 & \text { DLMT } \\ \text { 333WRSW } & 5,140 & 5,203 & \text { DLMT } \\ \text { 338OSGE } & 5,203 & 5,240 & \text { DLMT } \\ \text { 339KDRR } & 5,240 & 5,260 & \text { DMSH } \\ \text { 361VIOL } & 5,260 & 5,305 & \text { DLMT } \\ \text { 364SMPS } & 5,305 & 5,332 & \text { SNDS } \\ \text { 367ABCK } & 5,332 & 5,885 & \text { DLMT } \\ \text { 400PCMB } & 5,885 & & \text { GRNT }\end{array}$


Table 3.--Geologic logs for wells in the study area--Continued

Cleveland County, Oklahoma

Latitude: 351432

Owner: ROSE

Use of site: $P$

Altitude: 1,127
Long1tude: 973138

Local number: 09N-03W-21 BCA 1

Completion date:

Depth of well: 10,590

\begin{tabular}{|c|c|c|c|}
\hline $\begin{array}{l}\text { Geologic- } \\
\text { unit code }\end{array}$ & $\begin{array}{l}\text { Depth to } \\
\text { top }\end{array}$ & $\begin{array}{l}\text { Depth to } \\
\text { bottom }\end{array}$ & Lithology \\
\hline 112TRRCH & 0 & 30 & SAND \\
\hline 318LNRD & 30 & 1,570 & SDSL \\
\hline 319 WFMP & 1,570 & 2,860 & SDSL \\
\hline 322VRGL & 2,860 & 4,407 & SDSL \\
\hline 322VRGL & 4,407 & 4,470 & SNDS \\
\hline 322VRGL & 4,470 & 4,850 & SDSL \\
\hline 322VRGL & 4,850 & 4,890 & IMSN \\
\hline 322VRGL & 4,890 & 5,250 & SDSL \\
\hline 322VRGL & 5,250 & 5,305 & SDSL \\
\hline 323MSSR & 5,305 & 5,580 & IMSH \\
\hline 323MSSR & 5,580 & 5,860 & SNDS \\
\hline 323MSSR & 5,860 & 6,005 & SDSL \\
\hline 324BLCT & 6,005 & 6,045 & LMSN \\
\hline 323MSSR & 6,045 & 6,338 & SDSL \\
\hline 323MSSR & 6,338 & 6,515 & SDSL \\
\hline 323HGSR & 6,515 & 6,620 & LMSH \\
\hline 323MSSR & 6,620 & 6,775 & SHLE \\
\hline $323 \mathrm{CCKB}$ & 6,775 & 6,795 & IMSN \\
\hline 325MRMN & 6,795 & 7,670 & SHLE \\
\hline 325MRMN & 7,670 & 7,683 & IMSN \\
\hline 325CHRK & 7,683 & 8,240 & SDSL \\
\hline 325CHRK & 8,240 & 8,350 & LMSH \\
\hline 33OMSSP & 8,350 & 8,457 & SLSN \\
\hline 341WDFD & 8,457 & 8,608 & SHLE \\
\hline 34OHNTN & 8,608 & 8,893 & IMDM \\
\hline 357CMNL & 8,893 & 8,945 & IMSN \\
\hline 36ISLVN & 8,945 & 9,072 & SHLE \\
\hline 36IFRVL & 9,072 & 9,080 & LMSN \\
\hline 361 VIOL & 9,080 & 9,363 & IMSN \\
\hline 364BRMD & 9,363 & 9,464 & LMDM \\
\hline 364SMPS & 9,464 & 9,735 & SNDS \\
\hline 364MCLS & 9,735 & 9,850 & SLSH \\
\hline 364MCLS & 9,850 & 10,068 & SDSL \\
\hline 3640LCK & 10,068 & 10,330 & IMDM \\
\hline $367 \mathrm{ABCK}$ & 10,330 & & DLMT \\
\hline
\end{tabular}


Table 3.--Geologic logs for wells in the study area--Continued

Coal County, Oklahoma

Latitude: 343349

Owner: CRIM

Use of site: P

Altitude :
574
Longitude: 960953

Local number: 01N-11E-17 ABA 1

Completion date: 07/14/1972

Depth of well: 11,632

Geologic-

Depth to Depth to

unit code

top

bottom

Lithology

328WPCK

7,820

8,935

SHLE

327UNVL

8,935

327UNVL

8,960

333DLCK

3380GKK

341 WDFD

9,350

9,690

9,850

340HNTN

9,960

36ISLVN

10,040

36IVIOL

10,170

364SMPS

10,350

364SMPS

10,620

364SMPS

10,740

364SMPS

10,820

364SMPS

10,870

3640LCK

11,085

8,960

9,350

9,690

9,850

9,960

10,040

10,170

10,350

10,620

10,740

10,820

10,870

11,085

11,240

11,330

SNDS

SHLE

SHLE

IIMSN

SHLE

IMSN

SHLE

LMSN

LMSH

SDSL

I.MDM

SNDS

LMDM

11,240

11,440

SNDS

DLMT

I.MSN

IMSN

11,440 
Table 3.--Geologic logs for wells in the study area--Continued

Coal County, Oklahoma

Latitude: 344000

Owner: MORGAN

Use of site: $P$

Altitude: $\quad 725$
Longitude: 962036

Local number: 02N-09E-03 DC 1

Completion date: 09/13/1968

Depth of well: 8,038

\begin{tabular}{lrr|c}
$\begin{array}{l}\text { Geologic- } \\
\text { un1t code }\end{array}$ & $\begin{array}{r}\text { Depth } \\
\text { top }\end{array}$ & $\begin{array}{c}\text { Depth to } \\
\text { bottom }\end{array}$ & L1thology \\
324PSLVM & 0 & 580 & SDSL \\
324PSLVM & 580 & 2,400 & SHLE \\
325HRSR & 2,400 & 2,430 & SNDS \\
326ATCK & 2,430 & 5,560 & SHLE \\
328WPCK & 5,560 & 5,680 & LMSN \\
328WPCK & 5,680 & 5,860 & SHLE \\
327VNVL & 5,860 & 6,090 & SNDS \\
327VNVL & 6,090 & 6,470 & SHLE \\
333DLCK & 6,470 & 6,625 & SHLE \\
341WDFD & 6,625 & 6,710 & SHLE \\
340HNTN & 6,710 & 6,880 & LMSN \\
357CMNL & 6,880 & 6,910 & LMSN \\
361SLVN & 6,910 & 7,010 & SHLE \\
361VIOL & 7,010 & 7,090 & LMSN \\
364BRMD & 7,090 & 7,310 & LMDM \\
364BRMD & 7,310 & 7,425 & LMDM \\
364MCLS & 7,425 & 7,590 & DLMT \\
364MCLS & 7,590 & 7,710 & DLMT \\
364MCLS & 7,710 & 7,800 & SNDS \\
364MCLS & 7,800 & 7,820 & DLMT \\
3640LCK & 7,820 & 7,960 & SDSL \\
3640LCK & 7,960 & & SNDS
\end{tabular}

Comanche County, Oklahoma

Lat1tude: 344545

Owner: HUMBLE OIL Use of site: $P$ Altitude: 1,210
Longitude: 982218

Local number: 03N-11W-05 AD 1 Completion date: 10/15/1964

Depth of well: 1,591

\begin{tabular}{lrc|c}
$\begin{array}{l}\text { Geologic- } \\
\text { unit code }\end{array}$ & $\begin{array}{r}\text { Depth to } \\
\text { top }\end{array}$ & $\begin{array}{c}\text { Depth to } \\
\text { bottom }\end{array}$ & L1thology \\
318PSOK & 0 & 685 & ARKS \\
318PSOK & 685 & 1,410 & ARKS \\
370CMBR & 1,410 & & RYLT
\end{tabular}


Table 3.--Geologic logs for wells in the study area--Continued

Comanche County, Oklahoma

Latitude: 344626

Owner:

Use of site: P

Altitude: 1,272
Longitude: 981746

Local number: 04N-10W-31 CBA 1

Completion date: 11/11/1968

Depth of well: 6,603

$\begin{array}{lrrc}\begin{array}{l}\text { Geologic- } \\ \text { unit code }\end{array} & \begin{array}{r}\text { Depth to } \\ \text { top }\end{array} & \begin{array}{r}\text { Depth to } \\ \text { bottom }\end{array} & \text { L1thology } \\ \text { 318HNSS } & 50 & 250 & \text { SNDS } \\ \text { 318HNSS } & 250 & 521 & \text { SDSL } \\ \text { 318HNSS } & 521 & 760 & \text { SHLE } \\ \text { 318PSOK } & 760 & 1,060 & \text { CGLM } \\ \text { 318PSOK } & 1,060 & 1,150 & \text { SHLE } \\ \text { 340HNTN } & 1,150 & 1,490 & \text { LMSN } \\ \text { 361SLVN } & 1,490 & 1,820 & \text { SHLE } \\ \text { 361VIOL } & 1,820 & 2,420 & \text { LMSN } \\ \text { 361VKMK } & 2,420 & 2,890 & \text { LMSN } \\ \text { 364SMPS } & 2,890 & 3,020 & \text { LMSN } \\ \text { 364SMPS } & 3,020 & 3,100 & \text { SHLE } \\ \text { 364SMPS } & 3,100 & 3,420 & \text { SNDS } \\ \text { 364TPCK } & 3,420 & 3,660 & \text { SHLE } \\ \text { 364TPCK } & 3,660 & 3,760 & \text { LMSN } \\ \text { 364MCLS } & 3,760 & 3,890 & \text { SHLE } \\ \text { 364MCLS } & 3,890 & 4,050 & \text { LMSN } \\ \text { 364MCLS } & 4,050 & 4,220 & \text { SHLE } \\ \text { 364OLCK } & 4,220 & 4,320 & \text { LMSN } \\ \text { 364OLCK } & 4,320 & 4,810 & \text { SHLE } \\ \text { 364OLCK } & 4,810 & 5,225 & \text { LMSN } \\ \text { 367ABCK } & 5,225 & 6,050 & \text { DLMT } \\ \text { 367ABCK } & 6,050 & 6,250 & \text { LMSN } \\ \text { 364SMPS } & 6,250 & 6,500 & \text { LMSN } \\ \text { 364SMPS } & 6,500 & & \text { SHLE }\end{array}$


Table 3.--Geologic logs for wells in the study area--Continued

Craig County, Oklahoma

Latitude: 364526 Owner: HELMICK Use of site: T Altitude: $\quad 755$
Longitude: 950040

Local number: 26N-21E-12 BB 1 Completion date:

Depth of well: 1,773

\begin{tabular}{lrc|c}
$\begin{array}{l}\text { Geologic- } \\
\text { unit code }\end{array}$ & $\begin{array}{r}\text { Depth to } \\
\text { top }\end{array}$ & $\begin{array}{c}\text { Depth to } \\
\text { bottom }\end{array}$ & Lithology \\
367ABCK & 502 & 1,095 & SDMN \\
367RBDX & 1,095 & 1,165 & DLMT \\
367GSCDU & 1,165 & 1,210 & DLMT \\
367GSCDL & 1,210 & 1,430 & DLMT \\
367GNTR & 1,430 & 1,450 & SNDS \\
371EMCP & 1,450 & 1,594 & DLMT \\
371BNTR & 1,594 & 1,765 & DLMT \\
400PCMB & 1,765 & & GRNT
\end{tabular}


Table 3.--Geologic logs for wells in the study area--Continued

Craig County, Oklahoma

Latitude: 365016

Owner: NEILL Use of site: W Altitude: $\quad 900$
Longitude: 950705

Local number: $27 \mathrm{~N}-20 \mathrm{E}-12$ BDD 2 Completion date: 06/13/1971

Depth of well: 1,090

$\begin{array}{lrrc}\begin{array}{l}\text { Geologic- } \\ \text { unit code }\end{array} & \begin{array}{r}\text { Depth to } \\ \text { top }\end{array} & \begin{array}{c}\text { Depth to } \\ \text { bottom }\end{array} & \text { L1thology } \\ \text { 330BOON } & 550 & 638 & \text { LMSN } \\ \text { 339RDRK } & 638 & 650 & \text { SHLE } \\ \text { 339KDRK } & 650 & 660 & \text { LMSN } \\ \text { 341CTNG } & 660 & 680 & \text { SHLE } \\ \text { 367RBDX } & 680 & 790 & \text { DLMT } \\ \text { 367GSCD } & 790 & 950 & \text { DLMT } \\ \text { 400PCMB } & 950 & & \text { GRNT }\end{array}$

Craig County, Oklahoma

Latitude: 365000

Owner: RWD-3 Use of site: W Altitude :

876

Longitude: 950101

Local number: 27N-21B-12 CCB 1 Completion date: 07/07/1980

Depth of well: 1,352

$\begin{array}{lrrc}\begin{array}{l}\text { Geolog1c- } \\ \text { un1t code }\end{array} & \begin{array}{r}\text { Depth } \\ \text { top }\end{array} & \begin{array}{r}\text { Depth to } \\ \text { bottom }\end{array} & \text { L1thology } \\ \text { 325CHRK } & 140 & 230 & \text { SNDS } \\ \text { 332CSTR } & 230 & 260 & \text { LMSN } \\ \text { 333WRSW } & 260 & 410 & \text { LMSN } \\ \text { 3380SGE } & 410 & 500 & \text { LMDM } \\ \text { 338RSPG } & 500 & 620 & \text { LMSN } \\ \text { 339NRTV } & 620 & 627 & \text { SHLE } \\ \text { 330CTNG } & 627 & 670 & \text { SHLE } \\ \text { 367CTTR } & 670 & 840 & \text { DLMT } \\ \text { 368JFRC } & 840 & 1,015 & \text { DLMT } \\ \text { 367RBDX } & 1,015 & 1,210 & \text { DLMT } \\ \text { 367GSCDU } & 1,210 & & \text { DLMT }\end{array}$


Table 3.--Geologic logs for wells in the study area--Continued

Creek County, Oklahoma

Latitude: 355718 Owner: BERRYHILL Use of site: $P$ Altitude: $\quad 710$
Longitude: 960308

Local number: 17N-12E-17 ACA 1 Completion date:

Depth of well: 3,932

\begin{tabular}{lrr|l}
$\begin{array}{l}\text { Geologic- } \\
\text { unit code }\end{array}$ & $\begin{array}{r}\text { Depth } \\
\text { top }\end{array}$ & $\begin{array}{c}\text { Depth to } \\
\text { bottom }\end{array}$ & Lithology \\
323CFVL & 0 & 75 & SHLE \\
323SMNL & 75 & 175 & SHLE \\
325MRMN & 175 & 803 & SHLE \\
325CHRK & 803 & 1,447 & SDSL \\
325CHRK & 1,447 & 1,604 & SNDS \\
325CHRK & 1,604 & 1,792 & SDSL \\
326ATCK & 1,792 & 2,020 & SDSL \\
330MSSP & 2,020 & 2,158 & SHLE \\
330MSSP & 2,158 & 2,311 & LMSN \\
341CTNG & 2,311 & 2,318 & SHLE \\
364SMPS & 2,318 & 2,466 & SHLE \\
364SMPS & 2,466 & 2,560 & SNDS \\
367ABCK & 2,560 & 3,851 & DLMT \\
371GRNT & 3,851 & 3,924 & SDGL \\
400PCMB & 3,924 & & GRNT
\end{tabular}


Table 3.--Geologic logs for wells in the study area--Continued

Custer County, Oklahoma

Latitude: 354318

Owner: ARKLA

Use of site: P

Altitude: 1,795
Longitude: 990029

Local number: $14 \mathrm{~N}-17 \mathrm{~W}-04 \mathrm{~A} \quad 1$

Completion date: 05/12/1972

Depth of well: 16,700

\begin{tabular}{|c|c|c|c|}
\hline $\begin{array}{l}\text { Geologio- } \\
\text { unit code }\end{array}$ & $\begin{array}{c}\text { Depth to } \\
\text { top }\end{array}$ & $\begin{array}{l}\text { Depth to } \\
\text { bottom }\end{array}$ & Lithology \\
\hline 313BLIN & 600 & 700 & GPSM \\
\hline 310PRMN & 700 & 1,180 & SDSL \\
\hline 310PRMN & 1,180 & 1,800 & SHLE \\
\hline $310 \mathrm{PRMN}$ & 1,800 & 2,110 & SHLB \\
\hline 310PRMN & 2,110 & 2,650 & SHLE \\
\hline 310WLNG & 2,650 & 2,780 & GPSM \\
\hline 310WLNG & 2,780 & 3,050 & SHLE \\
\hline 310WLNG & 3,050 & 3,870 & ANDR \\
\hline 319CHSE & 3,870 & 4,230 & LMSN \\
\hline 319CHSE & 4,230 & 4,330 & SHLE \\
\hline 319CHSE & 4,330 & 4,430 & IMSN \\
\hline $319 \mathrm{CHSE}$ & 4,430 & 4,550 & SHLE \\
\hline 319CCGV & 4,550 & 4,800 & IMSN \\
\hline 319CCGV & 4,800 & 4,900 & SHLE \\
\hline 319CCGV & 4,900 & 4,970 & SNDS \\
\hline 319CCGV & 4,970 & 5,350 & SHLE \\
\hline 319CCGV & 5,350 & 5,400 & SNDS \\
\hline 319CCGV & 5,400 & 5,740 & SHLE \\
\hline 322VRGL & 5,740 & 6,280 & SHLE \\
\hline 322VRGL & 6,280 & 6,400 & SNDS \\
\hline 322VRGL & 6,400 & 7,380 & SHLE \\
\hline 322VRGL & 7,380 & 7,400 & SHLE \\
\hline 322VRGL & 7,400 & 7,660 & SHLE \\
\hline 321TRNT & 7.660 & 7,760 & LMSN \\
\hline 322VRGL & 7,760 & 7,910 & SDSI \\
\hline 322VRGL & 7,910 & 8,130 & SHLE \\
\hline 322DGLS & 8,130 & 8,280 & SNDS \\
\hline 323MSSR & 8,280 & 8,720 & SHLE \\
\hline 323LNSG & 8,720 & 8,860 & SHLE \\
\hline $323 \mathrm{KSSC}$ & 8,860 & 9,020 & SDSL \\
\hline $323 \mathrm{KSSC}$ & 9,020 & 9,190 & SHLE \\
\hline 323HGSR & 9,190 & 9,250 & SHLE \\
\hline 323KSSC & 9,250 & 9,370 & SDSL \\
\hline $323 \mathrm{KSSC}$ & 9,370 & 9,520 & SHLE \\
\hline 323CCKB & 9,520 & 9,600 & SHLE \\
\hline 323CFVL & 9,600 & 9,750 & SDSL \\
\hline 323CFVL & 9,750 & 9,870 & IMSH \\
\hline 325DSMS & 9,870 & 10,450 & SHLE \\
\hline 325CHRK & 10,450 & 10,610 & SDSI \\
\hline
\end{tabular}


Table 3.--Geologic logs for wells in the study area--Continued

Custer County, Oklahoma--Continued

Latitude: 354318

Owner: ARKLA

Use of site: $P$

Altitude: 1,795
Longitude: 990029

Local number: $14 \mathrm{~N}-17 \mathrm{~W}-04 \mathrm{~A} \quad 1$ Completion date: 05/12/1972

Depth of well: 16,700

\begin{tabular}{lrc|c}
$\begin{array}{l}\text { Geologic- } \\
\text { unit code }\end{array}$ & $\begin{array}{r}\text { Depth } \\
\text { top }\end{array}$ & $\begin{array}{c}\text { Depth to } \\
\text { bottom }\end{array}$ & Lithology \\
325CHRK & 10,610 & 10,760 & SHLE \\
325CHRK & 10,760 & 10,840 & LMSH \\
325CHRR & 10,840 & 11,125 & SHLE \\
325CHRK & 11,125 & 11,200 & LMSN \\
325CHRK & 11,200 & 11,690 & SHLE \\
328MRRW & 11,690 & 12,280 & SHLE \\
328MRRW & 12,280 & 12,640 & LMSN \\
328MRRW & 12,640 & 12,950 & SNDS \\
328SPRG & 12,950 & 13,390 & SHLE \\
332CSTR & 13,390 & 13,550 & IMSH \\
332CSTR & 13,550 & 13,940 & SHLE \\
332CSTR & 13,940 & 14,100 & IMSN \\
332CSTR & 14,100 & 14,620 & SHLE \\
330MSSP & 14,620 & 15,830 & LMSN \\
341WDFD & 15,830 & 15,950 & SHLE \\
340HNTN & 15,950 & 16,440 & DLMT \\
361SLVN & 16,440 & 16,500 & SHLE \\
361SLVN & 16,500 & 16,570 & DLMT \\
361VIOL & 16,570 & & LMSN
\end{tabular}


Table 3.--Geologic logs for we lls in the study area--Continued

Custer County, Oklahoma

Latitude: 354548

Owner: MACNOLIA

Use of site: $P$

Altitude: 1,794
Longitude: 985328

Local number: 15N-16W-22 BD 1

Completion date: 04/07/1959

Depth of well: 17,000

$\begin{array}{lccc}\begin{array}{l}\text { Geologio- } \\ \text { unit code }\end{array} & \begin{array}{r}\text { Depth } \\ \text { top }\end{array} & \begin{array}{c}\text { Depth to } \\ \text { bottom }\end{array} & \text { Lithology } \\ \text { 326ATCR } & 10,800 & 10,858 & \text { SHLE } \\ \text { 328MRRW } & 10,858 & 11,150 & \text { SHLE } \\ \text { 328MRRW } & 11,150 & 11,430 & \text { LMSH } \\ \text { 328MRRW } & 11,430 & 11,570 & \text { SNDS } \\ \text { 328SPRG } & 11,570 & 11,950 & \text { SHLE } \\ \text { 332CSTR } & 11,950 & 12,225 & \text { LMSN } \\ \text { 332CSTR } & 12,225 & 12,695 & \text { LMSH } \\ \text { 332CSTR } & 12,695 & 13,080 & \text { SHLE } \\ \text { 333MRMC } & 13,080 & 13,100 & \text { SLSN } \\ \text { 333MRMC } & 13,100 & 13,490 & \text { LMSN } \\ \text { 333MRMC } & 13,490 & 13,940 & \text { LMSN } \\ \text { 333MRMC } & 13,940 & 14,270 & \text { LMSN } \\ \text { 341WDFD } & 14,270 & 14,380 & \text { SHLE } \\ \text { 344HNTN } & 14,380 & 14,490 & \text { DLMT } \\ \text { 344HNTN } & 14,490 & 14,750 & \text { LMSN } \\ \text { 357CMNL } & 14,750 & 14,822 & \text { LMSN } \\ \text { 361SLVN } & 14,822 & 14,870 & \text { SHLE } \\ \text { 361SLVN } & 14,870 & 14,920 & \text { DLMT } \\ \text { 361VIOL } & 14,920 & 15,170 & \text { LMSN } \\ \text { 364BRMD } & 15,170 & 15,320 & \text { LMSN } \\ \text { 364BRMD } & 15,320 & 15,420 & \text { LMDM } \\ \text { 364BRMD } & 15,420 & 15,650 & \text { SNDS } \\ \text { 364BRMD } & 15,650 & 15,850 & \text { SHLE } \\ \text { 364BRMD } & 15,850 & 15,895 & \text { DLMT } \\ \text { 364BRMD } & 15,895 & 16,070 & \text { SHLE } \\ \text { 367ABCK } & 16,070 & 16,410 & \text { DLMT } \\ \text { 367ABCK } & 16,410 & 16,700 & \text { DLMT } \\ \text { 367ABCK } & 16,700 & & \text { DLMT }\end{array}$


Table 3.--Geologic logs for wells in the study area--Continued

Delaware County, Oklahoma

Latitude: 362442

Owner: RWD-1

Use of site: W

Altitude: 1,100
Longitude: 945208

Local number: 22N-23E-05 DCA 1

Completion date: $06 / 18 / 1979$

Depth of well: 1,538

\begin{tabular}{lrr|c}
$\begin{array}{c}\text { Geologic- } \\
\text { unit code }\end{array}$ & $\begin{array}{r}\text { Depth } \\
\text { top }\end{array}$ & $\begin{array}{c}\text { Depth to } \\
\text { bottom }\end{array}$ & Lithology \\
330MSSP & 0 & 185 & LMSH \\
341CTNG & 185 & 265 & SHLE \\
364EVRN & 265 & 300 & LMSN \\
368PWLI & 300 & 380 & DLMT \\
367CTTR & 380 & 675 & DLMT \\
368JFRC & 675 & 955 & DLMT \\
367RBDX & 955 & 1,110 & DLMT \\
367GSCD & 1,110 & 1,420 & DLMT \\
371EMNC & 1,420 & 1,515 & DLMT \\
400PCMB & 1,515 & & ARKS
\end{tabular}

Delaware County, Oklahoma

Latitude: 362832

Owner: COMMERCIAL

Use of site: T

Altitude: 1,045
Longitude: 943922

Local number: 23N-25E-17 BDB 1

Completion date: $01 / 01 / 1953$

Depth of well: 1,023

\begin{tabular}{lrr|c}
$\begin{array}{l}\text { Geologic- } \\
\text { unit code }\end{array}$ & $\begin{array}{r}\text { Depth to } \\
\text { top }\end{array}$ & $\begin{array}{r}\text { Depth to } \\
\text { bottom }\end{array}$ & Lithology \\
338FRGL & 0 & 15 & LMSN \\
341CTNG & 15 & 80 & SHLE \\
367CTTR & 80 & 445 & DLMT \\
368JFRC & 445 & 668 & DLMT \\
367RBDX & 668 & 830 & DLMT \\
367GSCDU & 830 & 892 & DLMT \\
367GSCDL & 892 & 1,018 & DLMT \\
400PCMB & 1,018 & & GRNT
\end{tabular}


Table 3.--Geologic logs for wells in the study area-Continued

Dewey County, Oklahoma

Lat1tude: 360821

Owner: MAGNOLIA

Use of site: $P$

Altitude: $\quad 2,225$
Longitude: 992007

Local number: 19N-20W-09 AC 1

Completion date: 09/28/1947

Depth of well: 12,856

\begin{tabular}{|c|c|c|c|}
\hline $\begin{array}{l}\text { Geologio- } \\
\text { unit code }\end{array}$ & $\begin{array}{c}\text { Depth to } \\
\text { top }\end{array}$ & $\begin{array}{l}\text { Depth to } \\
\text { bottom }\end{array}$ & Lithology \\
\hline 313ELRN & 310 & 620 & GPSM \\
\hline $313 F L R P$ & 620 & 900 & GPSM \\
\hline 310PRMN & 900 & 3,490 & SHLE \\
\hline 310PRMN & 3,490 & 3,840 & DMSH \\
\hline $319 W F M P$ & 3,840 & 4,000 & DLMT \\
\hline 319 WFMP & 4,000 & 4,390 & LMSN \\
\hline $319 W F M P$ & 4,390 & 4,680 & LMSH \\
\hline 319FRCK & 4,680 & 4,720 & LMSN \\
\hline 319 WFMP & 4,720 & 5,230 & IMSH \\
\hline 322VRGL & 5.230 & 5,370 & I.MSH \\
\hline 322VRGL & 5,370 & 5,670 & SDSL \\
\hline 322VRGL & 5,670 & 6,060 & IMSN \\
\hline 322VRGL & 6,060 & 6.850 & SDSL \\
\hline 322VRGL & 6,850 & 6,935 & SNDS \\
\hline 322VRGL & 6.935 & 7,350 & SHLE \\
\hline 322DGLS & 7.350 & 7,430 & SDSI \\
\hline 323MSSR & 7,430 & 8,410 & SHLE \\
\hline 325FRSC & 8,410 & 8,805 & LMSH \\
\hline 325FRSC & 8,805 & 9,100 & LMSH \\
\hline 326ATCK & 9,100 & 9,450 & SHLE \\
\hline 328MRRW & 9,450 & 10,090 & SHLE \\
\hline 328MRRW & 10,090 & 10,285 & SNDS \\
\hline 332CSTR & 10,285 & 11,390 & IMSN \\
\hline 332CSTR & 11,390 & 11,660 & SDSL \\
\hline 333MRMC & 11,660 & 12,285 & LMSN \\
\hline 333MRMC & 12,285 & 12,650 & LMSN \\
\hline 333MRMC & 12,650 & 12,925 & LMSH \\
\hline 341 WDFD & 12,925 & 12,955 & SHLE \\
\hline 340HNTN & 12,955 & 13,273 & DLMT \\
\hline 361SLVN & 13,273 & 13,300 & SHLE \\
\hline 361 SLVN & 13,300 & 13,377 & IMDM \\
\hline 361 VIOL & 13,377 & 13,732 & IMSN \\
\hline 364SMPS & 13,732 & & SNDS \\
\hline
\end{tabular}


Table 3.--Geologic logs for wells in the study area-Continued

Ellis County, Oklahona

Latitude: 355912 Owner: MCCULLOCH Use of site: $T$ Altitude: 2,395
Longitude: 994508

Local number: $18 \mathrm{~N}-24 \mathrm{~W}-34 \mathrm{CCA} 1$ Completion date: 03/12/1971

Depth of well: 17,500

\begin{tabular}{lrc|c}
$\begin{array}{l}\text { Geologic- } \\
\text { un1t code }\end{array}$ & $\begin{array}{r}\text { Depth } \\
\text { top }\end{array}$ & $\begin{array}{c}\text { Depth to } \\
\text { bottom }\end{array}$ & Lithology \\
322DGLS & 7,420 & 8,030 & SHLE \\
32OPSLV & 8,030 & 11,350 & SHLE \\
328MRRW & 11,350 & 11,933 & SHLE \\
328MRRW & 11,933 & 12,850 & SDSL \\
332CSTR & 12,850 & 13,050 & LMSN \\
33OMSSP & 13,050 & 14,415 & LMSH \\
330MSSP & 14,415 & 15,940 & LMSN \\
339KDKR & 15,940 & 15,960 & SHLE \\
341WDFD & 15,960 & 16,030 & SHLE \\
341WDFD & 15,960 & 16,030 & SHLE \\
34OHNTN & 16,030 & 16,364 & LMDM \\
361SLVN & 16,364 & 16,380 & DMSH \\
361VIOL & 16,380 & 16,910 & LMSN \\
364SMPS & 16,910 & 17,315 & SDSL \\
367ABCK & 17,315 & & DLMT
\end{tabular}


Table 3.--Geologic logs for wells in the study area-Continued

Ellis County, Oklahoma

Lat1tude: 363140

Owner: GULF OIL

Use of site: $T$

Altitude: 2,382
Long1tude: 995830

Local number: $24 \mathrm{~N}-26 \mathrm{~W}-27 \mathrm{CA} 1$

Completion date: 05/15/1944

Depth of well: 9,305

$\begin{array}{lrcc}\begin{array}{c}\text { Geologic- } \\ \text { unit code }\end{array} & \begin{array}{c}\text { Depth to } \\ \text { top }\end{array} & \begin{array}{c}\text { Depth to } \\ \text { bottom }\end{array} & \text { Lithology } \\ \text { 121OGLL } & 120 & 440 & \text { SAND } \\ \text { 313BLIN } & 440 & 578 & \text { GPSM } \\ \text { 313FLRP } & 578 & 860 & \text { EVPR } \\ \text { 31OPRMN } & 860 & 3,100 & \text { SHLE } \\ \text { 319WFMP } & 3,100 & 4,350 & \text { LMSN } \\ \text { 322WBNS } & 4,350 & 4,570 & \text { LMSN } \\ \text { 322VRGL } & 4,570 & 5,300 & \text { SHLE } \\ \text { 322ELGN } & 5,300 & 5,420 & \text { SNDS } \\ \text { 322SHWN } & 5,420 & 5,635 & \text { SHLE } \\ \text { 322SHWN } & 5,635 & 5,680 & \text { SNDS } \\ \text { 322DGLS } & 5,680 & 5,880 & \text { LMSH } \\ \text { 323HXBR } & 5,880 & 6,050 & \text { SHLE } \\ \text { 323HXBR } & 6,050 & 6,100 & \text { SNDS } \\ \text { 323HXBR } & 6,100 & 6,880 & \text { SHLE } \\ \text { 325DSMS } & 6,880 & 7,000 & \text { SHLE } \\ \text { 325DSMS } & 7,000 & 7,260 & \text { LMSN } \\ \text { 326ATCK } & 7,260 & 7,850 & \text { SHLE } \\ \text { 328MRRW } & 7,850 & 8,385 & \text { SHLE } \\ \text { 330MSSP } & 8,385 & 8,900 & \text { LMSN } \\ \text { 330MSSP } & 8,900 & 9,240 & \text { LMSH } \\ \text { 330MSSP } & 9,240 & & \text { SAND }\end{array}$


Table 3.--Geologic logs for wells in the study area--Continued

Garfield County, Oklahoma

Latitude: 362951

Owner: VOLIN

Use of site: $P$

Altitude: 1,050
Longitude: 973446

Local number: 23N-04W-01 CAA 1

Completion date:

Depth of well: 7,152

\begin{abstract}
Geologicunit code

318GRBR

31OWLNG

31 OWFMP

322WBNS

322WBNS

322WBNS

322SHWN

322DRCK

322 SHWN

322SHWN

322ORED

322 SHWN

322 SHWN

322DGLS

322DGLS

323MSSR

323MSSR

323MSSR

323K SSC

323MSSR

323HGSR

323MSSR

323 CCKB

325MRMN

325FRSC

$325 \mathrm{CHRK}$

330MSSP

341WDFD

361VIOL

364SMPS

364 SMPS

364SMPS

364SMPS

367ABCK
\end{abstract} Depth to
top $\begin{gathered}\text { Depth to } \\ \text { bottom }\end{gathered}$

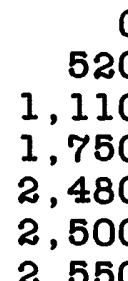

520

1,110

1,750

2,480

2,500

2,550

2,780

2,830

2,850

3,140

3,210

3,220

3,430

3,580

3,680

3,930

4,010

4,230

4,330

4,440

4,450

4,605

4,615

4,730

4,920

5,210

5,650

5,705

5,750

5,810

5,910

6,120

6,140
Lithology

SDSL

SHLE

IMSH

IMSH

IMSN

IMSH

SHLE

IMSH

SNDS

SHLE

LMSN

SHLE

SHLE

SDSL

SNDS

SHLE

SDSL

SHLE

SNDS

SHLE

SHLE

SHIE

LMSN

SHLE

LMSH

SHLE

IMSN

SHLE

LMSN

LMSH

SNDS

SDSL

DLMT

DIMT 
Table 3.--Geologic logs for wells in the study

area--Continued

Garvin County, Oklahoma

Latitude: 343139

Owner : HUMBLE OIL Use of site: $P$

Altitude: $\quad 857$
Longitude: 972018

Local number: 01N-01W-30 DAB 1 Completion date: 06/24/1971

Depth of well: 13,187

$\begin{array}{lrrc}\begin{array}{l}\text { Geologic- } \\ \text { unit code }\end{array} & \begin{array}{r}\text { Depth } \\ \text { top }\end{array} & \begin{array}{c}\text { Depth to } \\ \text { bottom }\end{array} & \text { Lithology } \\ \text { 319PNTC } & 50 & 540 & \text { SDSL } \\ \text { 319PNTC } & 540 & 960 & \text { LMSH } \\ \text { 319PNTC } & 960 & 1,170 & \text { CGLM } \\ \text { 319PNTC } & 1,170 & 2,460 & \text { CGLM } \\ \text { 319PNTC } & 2,460 & 3,420 & \text { CGLM } \\ \text { 367ABCK } & 3,420 & 7,300 & \text { LMSN } \\ \text { 367ABCK } & 7,300 & 8,700 & \text { LMSN } \\ \text { 367ABCK } & 8,700 & 9,230 & \text { LMSN } \\ \text { 367ABCK } & 9,230 & 9,450 & \text { DLMT } \\ \text { 367ABCK } & 9,450 & 9,900 & \text { LMSN } \\ \text { 367ABCK } & 9,900 & 10,670 & \text { DLMT } \\ \text { 367ABCK } & 10,670 & 10,970 & \text { SHLE } \\ \text { 367ABCK } & 10,970 & 11,230 & \text { LMSN } \\ \text { 371REGN } & 11,230 & 11,260 & \text { SNDS } \\ \text { 400PCMB } & 11,260 & 11,670 & \text { GRNT } \\ \text { 310PRMN } & 11,670 & 11,820 & \text { SNDS } \\ \text { 328SPRG } & 11,820 & 12,830 & \text { SHLE } \\ \text { 332CSTR } & 12,830 & 13,000 & \text { SHLE } \\ \text { 338SCMR } & 13,000 & & \text { LMSN }\end{array}$


Table 3.--Geologic logs for wells in the study area--Continued

Grady County, Oklahona

Lat1tude: 344547

Owner: PARKER

Use of site: P

Altitude: $\quad 1,123$
Longitude: 974029

Local number: 03N-05W-01 AC 1 Completion date: 11/01/1966

Depth of well: 18,413

\begin{tabular}{|c|c|c|c|}
\hline $\begin{array}{l}\text { Geologic- } \\
\text { unit code }\end{array}$ & $\underset{\text { top }}{\text { Depth to }}$ & $\begin{array}{l}\text { Depth to } \\
\text { bottom }\end{array}$ & Lithology \\
\hline 323MSSR & 6,600 & 6,910 & SHLE \\
\hline 324BLCT & 6,910 & 7,380 & LMSN \\
\hline 324BLCT & 7,380 & 7,805 & SHLE \\
\hline 323NLBL & 7,805 & 7,950 & SHLE \\
\hline 323HGSR & 7,950 & 8,460 & SHLE \\
\hline 325DEES & 8,460 & 9.020 & SHLE \\
\hline 325DEES & 9,020 & 9,135 & SDSL \\
\hline 325DEES & 9,135 & 9,300 & SHLE \\
\hline 325DEES & 9,300 & 9,395 & SDSL \\
\hline 325DEES & 9,395 & 9,505 & SDSL \\
\hline 325DEES & 9,505 & 9,715 & SHLE \\
\hline 325DEES & 9,715 & 9,985 & SDSL \\
\hline 328DCKH & 9,985 & 10,520 & SHLE \\
\hline 328DCKH & 10,520 & 10,665 & CGLM \\
\hline 328DCKH & 10,665 & 11,110 & SHLE \\
\hline 328DCKH & 11,110 & 11,430 & SDSI \\
\hline 328SPRG & 11,430 & 11,610 & SHLE \\
\hline 328SPRG & 11,610 & 11,770 & SNDS \\
\hline 328SPRG & 11,770 & 12,820 & SHLE \\
\hline 328SPRG & 12,820 & 12,920 & SHLE \\
\hline 328SPRG & 12,920 & 14,080 & SHLE \\
\hline 332CSTR & 14,080 & 14,160 & SHLE \\
\hline 333MRMC & 14,160 & 14,270 & LMSN \\
\hline 338SCMR & 14,270 & 14,425 & LMSN \\
\hline 34IWDFD & 14,425 & 14,710 & SHLE \\
\hline 340HNTN & 14,710 & 15,000 & LMSN \\
\hline 361SLVN & 15,000 & 15,230 & SHLE \\
\hline 361 VIOL & 15,230 & 15,500 & LMSN \\
\hline 361VIOL & 15,500 & 15,770 & IMSN \\
\hline 364BRMD & 15,770 & 15,850 & LMSN \\
\hline 364BRMD & 15,850 & 16,085 & SNDS \\
\hline 364BRMD & 16,085 & 16,220 & SDSL \\
\hline 364BRMD & 16,220 & 16,495 & SNDS \\
\hline 364BRMD & 16,495 & 16,590 & LMSH \\
\hline 364BRMD & 16,590 & 16,680 & SNDS \\
\hline 364OLCK & 16,680 & 17,080 & SHLE \\
\hline 3640LCK & 17,080 & 17,115 & LMSN \\
\hline 3640LCK & 17,115 & 17,190 & SNDS \\
\hline
\end{tabular}


Table 3.--Geologic logs for wells in the study area--Continued

Grady County, Oklahoma--Continued

Latitude: 344547

Owner: PARKER

Use of site: P

Altitude: 1,123
Longitude: 974029

Local number: 03N-05W-01 AC 1

Completion date: 11/01/1966

Depth of well: 18,413

$\begin{array}{lrrc}\begin{array}{r}\text { Geologic- } \\ \text { unit code }\end{array} & \begin{array}{r}\text { Depth to } \\ \text { top }\end{array} & \begin{array}{c}\text { Depth to } \\ \text { bottom }\end{array} & \text { Lithology } \\ \text { 364JONS } & 17,190 & 17,320 & \text { LMSN } \\ \text { 367ABCK } & 17,320 & 17,770 & \text { DLMT } \\ \text { 367ABCK } & 17,770 & 17,900 & \text { LMSN } \\ \text { 367ABCK } & 17,900 & 18,030 & \text { DLMT } \\ \text { 367ABCK } & 18,030 & 18,310 & \text { DLMT } \\ \text { 367ABCK } & 18,310 & & \text { LMSN }\end{array}$


Table 3.--Geologic logs for wells in the study area-Continued

Grady County, Oklahoma

Latitude: 350345

Owner: PHILLIPS

Use of site: $P$

Altitude: 1,161
Longitude: 980307

Local number: 07N-08W-21 CAC 1

Completion date: 10/29/1969

Depth of well: 10,754

\begin{tabular}{lrr|l}
$\begin{array}{l}\text { Geologio- } \\
\text { unit code }\end{array}$ & $\begin{array}{r}\text { Depth } \\
\text { top }\end{array}$ & $\begin{array}{r}\text { Depth to } \\
\text { bottom }\end{array}$ & Lithology \\
313ELRN & 0 & 410 & SHLE \\
313ELRN & 410 & 780 & SDSL \\
313ELRN & 780 & 1,350 & SHLE \\
313ELRN & 1,350 & 1,500 & SNDS \\
313ELRN & 1,500 & 2,230 & CLSN \\
313ELRN & 2,230 & 2,540 & SHLE \\
318WCHT & 2,540 & 3,690 & SHLE \\
319WFMP & 3,690 & 3,960 & SHLE \\
319WFMP & 3,960 & 4,680 & SHLE \\
319WFMP & 4,680 & 4,910 & SDSL \\
319WFMP & 4,910 & 5,290 & SHLE \\
319WFMP & 5,290 & 6,170 & SDSL \\
322VRGL & 6,170 & 7,615 & SHLE \\
322VRGL & 7,615 & 7,940 & SHLE \\
322ORED & 7,940 & 7,950 & LMSN \\
322VRGL & 7,950 & 8,700 & SHLE \\
322DGLS & 8,700 & 8,920 & SNDS \\
323MSSR & 8,920 & 9,180 & SHLE \\
323MSSR & 9,180 & 9,470 & SNDS \\
323MSSR & 9,470 & 9,660 & SHLE \\
323MSSR & 9,660 & 9,790 & SDSL \\
323MSSR & 9,790 & 10,088 & SHLE \\
323MSSR & 10,088 & 10,270 & SHLE \\
323HGSR & 10,270 & 10,390 & SHLE \\
323KSSC & 10,390 & & SDSL
\end{tabular}


Table 3.--Geologic logs for wells in the study area--Continued

Grant County, Oklahoma

Latitude: 365939 Owner: HENDRIXON Use of site: P Alt1tude: 1,271
Long1tude: 980513

Local number: 29N-08W-17 BDB 1 Completion date:

Depth of well: 5,660

$\begin{array}{lrrc}\begin{array}{c}\text { Geologic- } \\ \text { un1t code }\end{array} & \begin{array}{r}\text { Depth } \\ \text { top }\end{array} & \begin{array}{c}\text { Depth to } \\ \text { bottom }\end{array} & \text { L1thology } \\ \text { 318HNSS } & 0 & 100 & \text { SLSH } \\ \text { 318GRBR } & 100 & 900 & \text { SHLE } \\ \text { 310WLNG } & 900 & 1,350 & \text { SHLE } \\ \text { 310WLNG } & 1,350 & 1,480 & \text { ANDR } \\ \text { 319WFMP } & 1,480 & 2,700 & \text { LMSH } \\ \text { 322WBNS } & 2,700 & 2,940 & \text { LMSN } \\ \text { 322WBNS } & 2,940 & 3,100 & \text { SHLE } \\ \text { 322SHWN } & 3,100 & 3,340 & \text { LMSN } \\ \text { 322SHWN } & 3,340 & 3,440 & \text { SDSL } \\ \text { 322ORED } & 3,440 & 3,470 & \text { LMSN } \\ \text { 322SHWN } & 3,470 & 3,600 & \text { LMSN } \\ \text { 322DGLS } & 3,600 & 3,830 & \text { SHLE } \\ \text { 323MSSR } & 3,830 & 4,110 & \text { SDSL } \\ \text { 323MSSR } & 4,110 & 4,170 & \text { LMSN } \\ \text { 323MSSR } & 4,170 & 4,220 & \text { SNDS } \\ \text { 323MSSR } & 4,220 & 4,450 & \text { LMSN } \\ \text { 325MRMN } & 4,450 & 4,590 & \text { LMSN } \\ \text { 325CHRK } & 4,590 & 4,780 & \text { SHLE } \\ \text { 330MSSP } & 4,780 & 5,220 & \text { LMSN } \\ \text { 341WDFD } & 5,220 & 5,272 & \text { SHLE } \\ \text { 340MSNR } & 5,272 & 5,287 & \text { SNDS } \\ \text { 361VIOL } & 5,287 & 5,343 & \text { LMSN } \\ \text { 364SMPS } & 5,343 & 5,582 & \text { SNDS } \\ \text { 367ABCE } & 5,582 & & \text { DLMT }\end{array}$


Table 3.--Geologic logs for wells in the study area--Continued

Harper County, Oklahoma

Latitude: 364014

Owner: CONTINENTAL

Use of site: T

Altitude: $\quad 1,924$
Longitude: 993209

Local number: 25N-22W-02 DBD 1 Completion date: 09/10/1949

Depth of well: 9,440

$\begin{array}{lr}\begin{array}{l}\text { Geologic- } \\ \text { unit code }\end{array} & \begin{array}{r}\text { Depth } \\ \text { top }\end{array} \\ \text { 313MRLW } & 0 \\ \text { 313DGCK } & 90 \\ \text { 313BLIN } & 180 \\ \text { 310PRMN } & 350 \\ \text { 310PRMN } & 1,980 \\ \text { 319WFMP } & 2,760 \\ \text { 322WBNS } & 3,840 \\ \text { 322SHWN } & 4,415 \\ \text { 322DGLS } & 5,070 \\ \text { 322DGLS } & 5,215 \\ \text { 322DGLS } & 5,410 \\ \text { 323HXBR } & 5,440 \\ \text { 323HXBR } & 5,790 \\ \text { 325DSMS } & 6,050 \\ \text { 326ATCK } & 6,475 \\ \text { 328MRRW } & 6,660 \\ \text { 328MRRW } & 6,835 \\ \text { 332CSTR } & 6,920 \\ \text { 330MSSP } & 7,590 \\ \text { 340MSNR } & 8,770 \\ \text { 340HNTN } & 8,775 \\ \text { 361SLVN } & 8,930 \\ \text { 361VIOL } & 8,970 \\ \text { 364SMPS } & 9,020 \\ \text { 364SMPS } & 9,300 \\ \text { 364SMPS } & 9,365 \\ \text { 367ABCK } & 9,415\end{array}$

Depth to
bottom

$\begin{array}{r}90 \\ 180 \\ 350 \\ 1,980 \\ 2,760 \\ 3,840 \\ 4,415 \\ 5,070 \\ 5,215 \\ 5,410 \\ 5,440 \\ 5,790 \\ 6,050 \\ 6,475 \\ 6,660 \\ 6,835 \\ 6,920 \\ 7,590 \\ 8,770 \\ 8,775 \\ 8,930 \\ 8,970 \\ 9,020 \\ 9,300 \\ 9,365 \\ 9,415 \\ \hline\end{array}$

Lithology

SNDS

SHLE

GPSM

SHLE

ANDR

IMSN

IMSN

I.MSH

SDSL

SHLE

SNDS

SHLE

LMSH

LMSN

LMSH

SHLE

SNDS

LMSH

LMDM

SNDS

LMSN

IMDM

IMSN

DLMT

SHLE

SNDS

DLMT 
Table 3.--Geologic logs for wells in the study area-Continued

Haskell County, Oklahoma

Latitude: 350957

Owner: GORDON

Use of site: P

Altitude:

636
Longitude: 951138

Local number: 08N-20B-16 DBB 1 Completion date: 02/03/1955

Depth of well: 6,077

$\begin{array}{lrcc}\begin{array}{l}\text { Geologic- } \\ \text { unit code }\end{array} & \begin{array}{r}\text { Depth to } \\ \text { top }\end{array} & \begin{array}{c}\text { Depth to } \\ \text { bottom }\end{array} & \text { Lithology } \\ \text { 325MCAL } & 15 & 1,242 & \text { SHLE } \\ \text { 325HRSR } & 1,242 & 1,323 & \text { SNDS } \\ \text { 326ATCR } & 1,323 & 5,540 & \text { SHLE } \\ \text { 326ATCR } & 5,540 & 5,590 & \text { SNDS } \\ \text { 328WPCK } & 5,590 & 5,903 & \text { LMSH } \\ \text { 327UNVL } & 5,903 & 5,921 & \text { LMSH } \\ \text { 327UNVL } & 5,921 & & \text { SNDS }\end{array}$

Haskell County, Oklahoma

Latitude: 351010

Owner: ALLRED

Use of site: $P$

Altitude :

685
Longitude: 951345

Local number: 08N-20E-18 ADB 1 Completion date: $08 / 28 / 1951$

Depth of well: 7,483

$\begin{array}{lrrc}\begin{array}{c}\text { Geologic- } \\ \text { unit code }\end{array} & \begin{array}{r}\text { Depth } \\ \text { top }\end{array} & \begin{array}{c}\text { Depth to } \\ \text { bottom }\end{array} & \text { Lithology } \\ \text { 325MCAL } & 800 & 840 & \text { SNDS } \\ \text { 325MCAL } & 840 & 1,010 & \text { SHLE } \\ \text { 325MCAL } & 1,010 & 1,075 & \text { SNDS } \\ \text { 325MCAL } & 1,075 & 1,505 & \text { SHLE } \\ \text { 325HRSR } & 1,505 & 1,575 & \text { SNDS } \\ \text { 326ATCK } & 1,575 & 5,598 & \text { SHLE } \\ \text { 326ATCK } & 5,598 & 5,628 & \text { SNDS } \\ \text { 328WPCK } & 5,628 & 5,942 & \text { LMSH } \\ \text { 327UNVL } & 5,942 & 5,960 & \text { LMSN } \\ \text { 327UNVL } & 5,960 & 6,160 & \text { SNDS } \\ \text { 327UNVL } & 6,160 & 6,230 & \text { SHLE } \\ \text { 333DLCK } & 6,230 & 6,420 & \text { SHLE } \\ \text { 338OGKR } & 6,420 & 6,578 & \text { SHLE } \\ \text { 341WDFD } & 6,578 & 6,638 & \text { SHLE } \\ \text { 340HNTN } & 6,638 & 6,697 & \text { LMSN } \\ \text { 361SLVN } & 6,697 & 6,737 & \text { SHLE } \\ \text { 361VIOL } & 6,737 & 6,770 & \text { LMSN } \\ \text { 364BRMD } & 6,770 & 6,825 & \text { LMSN } \\ \text { 364BRMD } & 6,825 & 7,226 & \text { SNDS } \\ \text { 367ABCK } & 7,226 & & \text { DLMT }\end{array}$


Table 3.--Geologic logs for wells in the study area--Continued

Hughes County, Oklahoma

Lat1tude: 345529

Owner: PIERCE

Use of site: P

Altitude :

825
Longltude: 961838

Local number: 05N-09E-12 BAD 1

Completion date:

Depth of well: 6,957

\begin{tabular}{lrr|c}
$\begin{array}{l}\text { Geologic- } \\
\text { unit code }\end{array}$ & $\begin{array}{r}\text { Depth to } \\
\text { top }\end{array}$ & $\begin{array}{r}\text { Depth to } \\
\text { bottom }\end{array}$ & L1thology \\
325DEES & 0 & 330 & SDSL \\
325DEES & 330 & 1,100 & SHLE \\
325DEES & 1,100 & 1,450 & SDSL \\
325DEES & 1,450 & 1,670 & SHLE \\
325DEES & 1,670 & 2,830 & SDSL \\
325HRSR & 2,830 & 2,860 & SNDS \\
326ATOK & 2,860 & 3,450 & SHLE \\
326ATOK & 3,450 & 3,860 & SDSL \\
328WPCK & 3,860 & 3,930 & LMSN \\
328MRRW & 3,930 & 4,315 & SHLE \\
328MRRW & 4,315 & 4,460 & SDSL \\
328MRRW & 4,460 & 4,670 & SHLE \\
332CSTR & 4,670 & 4,800 & SHLE \\
332CSTR & 4,800 & 5,040 & LMSN \\
333MRMC & 5,040 & 5,155 & LMSN \\
341WDFD & 5,155 & 5,320 & SHLE \\
34OHNTN & 5,320 & 5,340 & LMSN \\
361SLVN & 5,340 & 5,440 & SHLE \\
361FRVL & 5,440 & 5,480 & LMSN \\
361VIOL & 5,480 & 5,510 & LMSN \\
364SMPS & 5,510 & 5,860 & DLMT \\
364SMPS & 5,860 & 6,000 & SDSL \\
364OLCK & 6,000 & 6,385 & SNDS \\
367ABCK & 6,385 & & DLMT
\end{tabular}


Table 3.--Geologic logs for wells in the study area--Cont1nued

Johnston County, Oklahoma

Latitude: 341328

Owner: CHAPMAN

Use of site: $P$

Altitude:
655
Longitude: 964912

Local number: 04S-05E-07 BDD 1

Completion date: $04 / 15 / 1979$

Depth of well: 11,747

$\begin{array}{lrcc}\begin{array}{l}\text { Geologic- } \\ \text { unit code }\end{array} & \begin{array}{r}\text { Depth to } \\ \text { top }\end{array} & \begin{array}{c}\text { Depth to } \\ \text { bottom }\end{array} & \text { Lithology } \\ \text { 328SPRG } & 70 & 1,200 & \text { SHLE } \\ \text { 328SPRG } & 1,200 & 2,730 & \text { SHLE } \\ \text { 333DLCK } & 2,730 & 2,960 & \text { SHLE } \\ \text { 333DLCK } & 2,960 & 3,070 & \text { LMSH } \\ \text { 338SCMR } & 3,070 & 3,220 & \text { LMSN } \\ \text { 338SCMR } & 3,220 & 3,300 & \text { LMSH } \\ \text { 341WDFD } & 3,300 & 3,630 & \text { SHLE } \\ \text { 361SLVN } & 3,630 & 3,810 & \text { SHLE } \\ \text { 361SLVN } & 3,810 & 3,920 & \text { SHLE } \\ \text { 361VIOL } & 3,920 & 4,670 & \text { LMSN } \\ \text { 364SMPS } & 4,670 & 5,070 & \text { LMSH } \\ \text { 364SMPS } & 5,070 & 5,160 & \text { SNDS } \\ \text { 364SMPS } & 5,160 & 5,720 & \text { LMSH } \\ \text { 364MCLS } & 5,720 & 5,840 & \text { SNDS } \\ \text { 364OLCK } & 5,840 & 5,990 & \text { LMSH } \\ \text { 364OLCK } & 5,990 & 6,540 & \text { SHLE } \\ \text { 364OLCK } & 6,540 & 6,600 & \text { LMSN } \\ \text { 364OLCK } & 6,600 & 6,800 & \text { SNDS } \\ \text { 364OLCK } & 6,800 & 7,050 & \text { LMSN } \\ \text { 367ABCK } & 7,050 & 7,700 & \text { LMDM } \\ \text { 367ABCK } & 7,700 & 8,530 & \text { LMSN } \\ \text { 367ABCK } & 8,530 & 8,680 & \text { LMDM } \\ \text { 367ABCK } & 8,680 & & \text { LMSN }\end{array}$


Table 3.--Geologic logs for wells in the study area-Continued

Latitude: 365414

Owner: WELCH

Use of site: P

Altitude: 1,138

Kay County, Oklahoma

\begin{tabular}{lrr|c}
$\begin{array}{l}\text { Geologic- } \\
\text { unit code }\end{array}$ & $\begin{array}{r}\text { Depth to } \\
\text { top }\end{array}$ & $\begin{array}{c}\text { Depth to } \\
\text { bottom }\end{array}$ & Lithology \\
310WLNG & 0 & 890 & SHLE \\
322VRGL & 890 & 1,495 & SHLE \\
322VRGL & 1,495 & 1,605 & LMSN \\
322VRGL & 1,605 & 2,150 & SHLE \\
323TLNT & 2,150 & 2,296 & SNDS \\
323MSSR & 2,296 & 2,610 & SHLE \\
323MSSR & 2,610 & 2,695 & SNDS \\
323MSSR & 2,695 & 2,990 & SHLE \\
325DSMS & 2,990 & 3,132 & LMSN \\
325DSMS & 3,132 & 3,196 & SHLE \\
367ABCK & 3,196 & & DLMT
\end{tabular}

Longitude: 971324

Local number: 28N-01E-17 CAB 1 Completion date:

Depth of well: 4,407 
Table 3.--Geologic logs for wells in the study area--Continued

Kingfisher County, Oklahoma

Lat1tude: 355619

Owner: ASH

Use of site: P

Altitude: 1,005
Longitude: $\quad 974613$

Local number: 17N-05W-19 ABD 1

Completion date:

Depth of well: 8,519

\begin{tabular}{|c|c|c|c|}
\hline $\begin{array}{l}\text { Geologic- } \\
\text { unit code }\end{array}$ & $\underset{\text { Depth }}{\text { to }}$ & $\begin{array}{l}\text { Depth to } \\
\text { bottom }\end{array}$ & L1thology \\
\hline $\begin{array}{l}\text { 31OPRMN } \\
\text { 31OPRMN } \\
\text { 322VRGL } \\
\text { 322ORED } \\
\text { 322SHWN } \\
\text { 321TRNT } \\
\text { 322DGLS } \\
\text { 322DGLS } \\
\text { 323INSG } \\
\text { 323LNSG } \\
\text { 323MSSR } \\
\text { 323MSSR } \\
\text { 323MSSR } \\
\text { 323CCKB } \\
\text { 323CFVI } \\
\text { 325MRMN } \\
\text { 325CHRK } \\
\text { 333MRMC } \\
\text { 338OSGE } \\
\text { 341WDFD } \\
\text { 340HNTN } \\
\text { 361SLVN } \\
\text { 361VIOL } \\
\text { 364SMPS } \\
\text { 364SMPS } \\
\text { 367ABCK }\end{array}$ & $\begin{array}{l}0 \\
1,670 \\
3,480 \\
4,197 \\
4,270 \\
4,420 \\
4,470 \\
4,660 \\
4,855 \\
4,855 \\
5,110 \\
5,500 \\
5,590 \\
5,879 \\
5,914 \\
6,162 \\
6,357 \\
6,692 \\
6,990 \\
7,200 \\
7,260 \\
7,452 \\
7,550 \\
7,610 \\
7,870 \\
8,400\end{array}$ & $\begin{array}{l}1,670 \\
3,480 \\
4,197 \\
4,270 \\
4,420 \\
4,470 \\
4,660 \\
4,855 \\
5,040 \\
5,110 \\
5,500 \\
5,590 \\
5,879 \\
5,914 \\
6,162 \\
6,357 \\
6,692 \\
6,990 \\
7,200 \\
7,260 \\
7,452 \\
7,550 \\
7,610 \\
7,870 \\
8,400\end{array}$ & $\begin{array}{l}\text { SHLE } \\
\text { SHLE } \\
\text { SDSL } \\
\text { LMSN } \\
\text { SDSL } \\
\text { LMSN } \\
\text { SHLE } \\
\text { SDSL } \\
\text { SHLE } \\
\text { SDSL } \\
\text { SHLE } \\
\text { SLSN } \\
\text { SHLE } \\
\text { SHLE } \\
\text { SHLE } \\
\text { LMSN } \\
\text { SHLE } \\
\text { SLSN } \\
\text { LMSN } \\
\text { SHLE } \\
\text { LMSN } \\
\text { SHLE } \\
\text { LMSN } \\
\text { LMDM } \\
\text { SNDS } \\
\text { DLMT }\end{array}$ \\
\hline
\end{tabular}


Table 3.--Geologic logs for wells in the study area--Continued

Kingfisher County, Oklahoma

Latitude: 360132

Owner: VILHAUER

Use of site: $P$

Altitude: 1,188
Longitude: 980903

Local number: 18N-09W-22 BAC 1

Completion date:

Depth of well: $\quad 9,639$
Geologicunit code

313FLRP

318 HNSS

318GRBR

31OWLNG

31OWLNG

3IONLNS

319CHSE

317WFLD

319CHSE

317BRNS

319CHSE

317WRFD

319CCGV

319CCGV

319CCGV

319GRNL

319CCGV

319CCGV

319ADMR

322WBNS

322WBNS

322WBNS

322WBNS

322TOPK

322SHWN

322SHWN

322SHWN

322SHWN

322ORED

322DGLS

323LNSG

323KSSC

323MSSR

323HGSR

323MSSR

323 PLSN

325MRMN

325CBNS
Depth to
top

40
610
1,405
1,733
2,428
2,474
2,480
2,520
2,564
2,710
2,730
2,812
2,893
3,019
3,073
3,155
3,278
3,400
3,990
4,150
4,268
4,333
4,490
4,550
4,747
4,902
5,122
5,260
5,397
5,647
5,775
6,187
6,451
6,453
6,470
6,885
7,180
7,454

Lithology

SHLE

SHLE

SDSI

SHLE

GPSM

DLMT

SHLE

IMDM

SHLE

IMSN

SHLE

IMSN

SHLE

IMSN

SHLE

IMSN

SHLE

LMSN

SHLE

LMSN

SHLE

IMSN

SHLE

LMSN

SHLE

SDSI

SHLE

SDSL

IMSN

SHLE

SDSL

SHLE

SDSI

IMSN

SHLE

SDSL

IMSN

SHLE 
Table 3.--Geologic logs for wells in the study area-Continued

Kingfisher County, Oklahoma--Continued

Latitude: 360132

Owner: VILHAUER

Use of site: P

Altitude: 1,188
Longitude : 980903

Local number: 18N-09W-22 BAC 1 Completion date:

Depth of well: $\quad 9,639$

$\begin{array}{lrrr}\begin{array}{c}\text { Geologic- } \\ \text { unit code }\end{array} & \begin{array}{r}\text { Depth to } \\ \text { top }\end{array} & \begin{array}{c}\text { Depth to } \\ \text { bottom }\end{array} & \text { Lithology } \\ \text { 332CSTR } & 7,454 & 7,608 & \text { IMSN } \\ \text { 332CSTR } & 7,608 & 8,076 & \text { SHLE } \\ \text { 330MSSP } & 8,076 & 8,594 & \text { LMSN } \\ \text { 339RDRR } & 8,594 & 8,598 & \text { SHLE } \\ \text { 341WDFD } & 8,598 & 8,627 & \text { SHLE } \\ \text { 340HNTN } & 8,627 & 8,690 & \text { IMDM } \\ \text { 340HNTN } & 8,690 & 9,058 & \text { LMSN } \\ \text { 361SLVN } & 9,058 & 9,160 & \text { SHLE } \\ \text { 361VIOL } & 9,160 & 9,225 & \text { IMSN } \\ \text { 364BRMD } & 9,225 & 9,431 & \text { IMDM } \\ \text { 364BRMD } & 9,431 & 9,603 & \text { SNDS } \\ \text { 364SMPS } & 9,603 & & \text { SHLE }\end{array}$


Table 3.--Geologic logs for wells in the study area-Continued

Kiowa County, Oklahoma

Latitude: 350539

Owner: EASON OIL Use of site: $P$

Altitude: $\quad 1,689$
Iongitude: 991400

Local number: 07N-19W-08 CBA 1

Completion date: $11 / 26 / 1976$

Depth of well: 4,296

\begin{tabular}{lrc|c}
$\begin{array}{l}\text { Geologic- } \\
\text { unit code }\end{array}$ & $\begin{array}{r}\text { Depth } \\
\text { top }\end{array}$ & $\begin{array}{r}\text { Depth to } \\
\text { bottom }\end{array}$ & Lithology \\
310PRMN & 400 & 1,340 & SHLE \\
319PNTC & 1,340 & 1,430 & SHLE \\
319PNTC & 1,430 & 1,690 & SHLE \\
319PNTC & 1,690 & 2,430 & ARKS \\
319PNTC & 2,430 & 3,080 & CGLM \\
319PNTC & 3,080 & 3,370 & CGLM \\
319PNTC & 3,370 & 3,430 & CGLM \\
319PNTC & 3,430 & 3,640 & CGLM \\
319PNTC & 3,640 & 3,680 & ARKS \\
319PNTC & 3,680 & 3,810 & CGLM \\
319PNTC & 3,810 & 4,160 & ARKS \\
370CMBR & 4,160 & & GRNT
\end{tabular}

Latimer County, Oklahoma

Latitude: 345706

Owner: FIELDS

Use of site: $P$

Alt1tude: $\quad 646$
Longitude: 950120

Local number: 06N-22E-31 BDB 1

Completion date: 04/08/1977

Depth of well: 12,350

\begin{tabular}{lrc|c}
$\begin{array}{l}\text { Geologic- } \\
\text { unit code }\end{array}$ & $\begin{array}{r}\text { Depth to } \\
\text { top }\end{array}$ & $\begin{array}{c}\text { Depth to } \\
\text { bottom }\end{array}$ & Lithology \\
325KRBS & 310 & 2,180 & SHLE \\
325HRSR & 2,180 & 2,530 & SDSL \\
326ATCK & 2,530 & 4,300 & SHLE \\
326ATCK & 4,300 & 4,890 & SHLE \\
326ATCK & 4,890 & 5,960 & SHLE \\
326ATCK & 5,960 & 6,070 & SDSL \\
326ATCK & 6,070 & 12,240 & SHLE \\
325SVNN & 12,240 & 12,310 & SNDS \\
325SVNN & 12,310 & & SHLE
\end{tabular}


Table 3.--Geologic logs for wells in the study area-Continued

Leflore County, Oklahoma

Latitude: 351010

Owner: HICKMAN

Use of site: P

Altitude: $\quad 465$
Longltude: 944115

Local number: 08N-25E-17 BDB 1

Completion date: $01 / 29 / 1952$

Depth of well: 7,650

\begin{tabular}{|c|c|c|c|}
\hline $\begin{array}{l}\text { Geologic- } \\
\text { unit code }\end{array}$ & $\begin{array}{c}\text { Depth to } \\
\text { top }\end{array}$ & $\begin{array}{l}\text { Depth to } \\
\text { bottom }\end{array}$ & Lithology \\
\hline $\begin{array}{l}\text { 326ATCK } \\
\text { 326ATCK } \\
\text { 326ATCK } \\
\text { 326ATCK }\end{array}$ & $\begin{array}{r}0 \\
2,520 \\
2,680 \\
2,970\end{array}$ & $\begin{array}{l}2,520 \\
2,680 \\
2,970\end{array}$ & $\begin{array}{l}\text { SHLE } \\
\text { SNDS } \\
\text { SDSL } \\
\text { SHLE }\end{array}$ \\
\hline
\end{tabular}

Leflore County, Oklahoma

Lat1tude: 351128

Owner: COLEMAN

Use of site: $P$

Altitude :
419
Longitude: 943503

Local number: 08N-26E-05 CCA 1

Completion date: 11/21/1974

Depth of well: 8,841

$\begin{array}{lccc}\begin{array}{c}\text { Geologic- } \\ \text { un1t code }\end{array} & \begin{array}{c}\text { Depth } \\ \text { top }\end{array} & \begin{array}{c}\text { Depth to } \\ \text { bottom }\end{array} & \text { Lithology } \\ \text { 326ATCK } & 7,400 & 7,480 & \text { SHLE } \\ \text { 326ATCK } & 7,480 & 7,530 & \text { SDSL } \\ \text { 326ATCK } & 7,530 & 7,580 & \text { SHLE } \\ \text { 328WPCK } & 7,580 & 7,790 & \text { LMSH } \\ \text { 328WPCK } & 7,790 & 7,950 & \text { SHLE } \\ \text { 327UNVL } & 7,950 & 7,990 & \text { LMSN } \\ \text { 327UNVL } & 7,990 & 8,080 & \text { LMSN } \\ \text { 327UNVL } & 8,080 & 8,210 & \text { LMSN } \\ \text { 327UNVL } & 8,210 & 8,480 & \text { SHLE } \\ \text { 341WDFD } & 8,480 & 8,628 & \text { SHLE } \\ \text { 361 SLVN } & 8,628 & 8,658 & \text { LMSH } \\ \text { 361VIOL } & 8,658 & & \text { LMSN }\end{array}$


Table 3.--Geologic logs for wells in the study

area--Continued

Lincoln County, Oklahoma.

Latitude: 353528

Owner: LINAN

Use of site: P

Altitude: $\quad 1,038$
Longitude: 970039

Local number: 13N-03E-20 BAC 1 Completion date:

Depth of well: 6,350

\begin{tabular}{lrc|c}
$\begin{array}{l}\text { Geologic- } \\
\text { un1t code }\end{array}$ & $\begin{array}{r}\text { Depth to } \\
\text { top }\end{array}$ & $\begin{array}{r}\text { Depth to } \\
\text { bottom }\end{array}$ & Lithology \\
320PSLV & 0 & 3,000 & SDSL \\
323MSSR & 3,000 & 3,180 & SHLE \\
323MSSR & 3,180 & 3,240 & LMSN \\
323MSSR & 3,240 & 3,428 & SDSL \\
323HGSR & 3,428 & 3,450 & LMSN \\
323MSSR & 3,450 & 3,537 & SHLE \\
323CCKB & 3,537 & 3,575 & LMSN \\
323MSSR & 3,575 & 3,785 & SHLE \\
323CCKB & 3,785 & 3,800 & LMSN \\
325MRMN & 3,800 & 4,435 & SHLE \\
325MRMN & 4,436 & 4,475 & LMSN \\
325CHRK & 4,475 & 4,575 & SNDS \\
325CHRK & 4,575 & 4,732 & SHLE \\
325CHRK & 4,732 & 4,878 & SHLE \\
325CHRK & 4,878 & 4,965 & SHLE \\
325CHRK & 4,965 & 5,058 & SHLE \\
330MSSP & 5,058 & 5,140 & LMSN \\
341WDFD & 5,140 & 5,260 & SHLE \\
340HNTN & 5,220 & 5,270 & DLMT \\
361SLVN & 5,270 & 5,360 & SHLE \\
361FRVI & 5,360 & 5,430 & LMSN \\
364BRMD & 5,430 & 5,493 & LMDM \\
364SMPS & 5,493 & 5,565 & DLMT \\
364SMPS & 5,565 & 5,990 & SNDS \\
364SMPS & 5,990 & 6,130 & SDSL \\
367ABCK & 6,130 & & DLMT
\end{tabular}


Table 3.--Geologic logs for wells in the study area--Continued

Lincoln County, Oklahoma

Latitude: 354040

Owner: CUNNINGHAM

Use of site: P

Altitude :

933
Longitude: 965221

Local number: 14N-04E-22 BCA 1

Completion date:

Depth of well: 5,024

$\begin{array}{lrrc}\begin{array}{l}\text { Geologic- } \\ \text { unit code }\end{array} & \begin{array}{r}\text { Depth } \\ \text { top }\end{array} & \begin{array}{r}\text { Depth to } \\ \text { bottom }\end{array} & \text { Lithology } \\ \text { 322VNSS } & 0 & 490 & \text { SHLE } \\ \text { 322VRGL } & 490 & 830 & \text { SDSL } \\ \text { 323MSSR } & 830 & 905 & \text { LMSH } \\ \text { 323MSSR } & 905 & 1,530 & \text { SHLE } \\ \text { 323MSSR } & 1,530 & 1,890 & \text { SDSL } \\ \text { 323MSSR } & 1,890 & 2,390 & \text { SDSL } \\ \text { 323MSSR } & 2,390 & 2,420 & \text { LMSN } \\ \text { 323MSSR } & 2,420 & 2,780 & \text { SDSL } \\ \text { 323HGSR } & 2,780 & 2,820 & \text { LMSN } \\ \text { 323MSSR } & 2,820 & 2,980 & \text { LMSH } \\ \text { 323MSSR } & 2,980 & 3,110 & \text { SHLE } \\ \text { 323CCKB } & 3,110 & 3,140 & \text { LMSN } \\ \text { 323MSSR } & 3,140 & 3,160 & \text { SNDS } \\ \text { 325MRMN } & 3,160 & 3,240 & \text { LMSN } \\ \text { 325MRMN } & 3,240 & 3,820 & \text { SHLE } \\ \text { 325MRMN } & 3,820 & 3,870 & \text { LMSH } \\ \text { 325CHRK } & 3,870 & 3,950 & \text { SNDS } \\ \text { 325CHRR } & 3,950 & 4,010 & \text { LMSN } \\ \text { 325CHRR } & 4,010 & 4,140 & \text { SHLE } \\ \text { 325CHRK } & 4,140 & 4,160 & \text { LMSN } \\ \text { 325CHRR } & 4,160 & 4,195 & \text { SHLE } \\ \text { 325CHRR } & 4,195 & 4,220 & \text { SNDS } \\ \text { 325CHRR } & 4,220 & 4,275 & \text { SHLE } \\ \text { 325CHRR } & 4,275 & 4,430 & \text { LMSH } \\ \text { 325CHRR } & 4,430 & 4,530 & \text { SDSL } \\ \text { 330MSSP } & 4,530 & 4,750 & \text { LMSN } \\ \text { 341WDFD } & 4,750 & 4,803 & \text { SHLE } \\ \text { 340HNTN } & 4,803 & 4,850 & \text { DLMT } \\ \text { 361SLVN } & 4,850 & 4,950 & \text { SHLE } \\ \text { 361VIOL } & 4,950 & 4,990 & \text { LMSN } \\ \text { 364BRMD } & 4,990 & 5,000 & \text { LMSN } \\ \text { 364SMPS } & 5,000 & & \text { DLMT } \\ & & & \\ & & & \end{array}$


Table 3.--Geologic logs for wells in the study area--Continued

Logan County, Oklahoma

Latitude: 354629

Owner: KROUT

Use of site: P

Altitude: $\quad 1,006$
Longitude: 973554

Local number: 15N-04W-14 CBA 1 Completion date:

Depth of well: 8,350
Geologic-

unit code

318GRBR

31OPRMN

$310 P M P V$

322WBNS

322WBNS

322TOPK

322 SHWN

322 SHWN

322 SHWN

322 SHWN

322ORED

322 SHWN

322DGLS

322DGLS

322DGLS

323MSSR

323MSSR

323MSSR

323MSSR

323MSSR

323HGSR

323MSSR

323CCKB

323MSSR

323CCKB

323MSSR

325MRMN

325MRMN

325MRMN

325CHRE

361 SLVN

361FRVL

36IVIOL

364SMPS

364SMPS

364MCLS

364OLCK

364JONS

367ABCK
Depth to
top bottom

0

440

1,250

2,940

2, 980

3,190

3,215

3,428

3,460

3,475

3,938

3,970

4,120

4,248

4,308

4,340

4,610

4,730

4,985

5,010

5,090

5,112

5,190

5,250

5,368

5,380

5,400

5,660

5,770

5,893

6,017

6,095

6,130

6,228

6,450

6,660

6,782

7,225

7,258
Lithology

SDSL

SDSL

SHLE

LMSN

SDSL

IMSH

SDSL

SNDS

SHLE

SDSL

SHLE

SDSL

SDSL

LMSH

SNDS

SDSL

LMSH

SHLE

SNDS

SHLE

LMSN

SNDS

IMSN

IMSH

LMSN

SNDS

SHLE

SDSL

LMSH

SHLE

SHLE

IMSN

LMSN

DLMT

SNDS

SHLE

SDSL

DLMT

DLMT 
Table 3.--Geologic logs for wells in the study area--Continued

MoClain County, Oklahoma

Latitude: 345956

Owner: LITTLE Use of site: $P$ Altitude: 1,134
Longitude: 972342

Local number: 06N-02W-15 AB 1 Completion date: 07/08/1971

Depth of well: 10,037

$\begin{array}{lrrc}\begin{array}{c}\text { Geologic- } \\ \text { unit code }\end{array} & \begin{array}{r}\text { Depth } \\ \text { top }\end{array} & \begin{array}{c}\text { Depth to } \\ \text { bottom }\end{array} & \text { Lithology } \\ \text { 318HNSS } & 30 & 690 & \text { SHLE } \\ \text { 318HNSS } & 690 & 1,060 & \text { SDSL } \\ \text { 310WLNG } & 1,060 & 2,470 & \text { SHLE } \\ \text { 319PNTC } & 2,470 & 3,935 & \text { SHLE } \\ \text { 320PSLV } & 3,935 & 7.400 & \text { SHLE } \\ \text { 341WDFD } & 7,400 & 7,560 & \text { SHLE } \\ \text { 340HNTN } & 7,560 & 7,890 & \text { LMSN } \\ \text { 361SLVN } & 7,890 & 8,020 & \text { SHLE } \\ \text { 361VIOL } & 8,020 & 8,350 & \text { LMSN } \\ \text { 364SMPS } & 8,350 & 8,510 & \text { LMSN } \\ \text { 364SMPS } & 8,510 & 8,710 & \text { SNDS } \\ \text { 364SMPS } & 8,710 & 9,170 & \text { SDSL } \\ \text { 364OLCK } & 9,170 & 9,520 & \text { SDSL } \\ \text { 367ABCK } & 9,520 & 9,850 & \text { DLMT } \\ \text { 367ABCK } & 9,850 & & \text { LMSN }\end{array}$


Table 3.--Geologic logs for wells in the study area--Continued

McIntosh County, Oklahoma

Latitude: 351503

Owner: FOLLANSBEE

Use of site: P Altitude :
653
Longitude: 954538

Local number: 09N-15E-18 CBC 1

Completion date: $11 / 28 / 1950$

Depth of well: 5,461

$\begin{array}{lrrr}\begin{array}{l}\text { Geologic- } \\ \text { un1t code }\end{array} & \begin{array}{r}\text { Depth } \\ \text { top }\end{array} & \begin{array}{r}\text { Depth to } \\ \text { bottom }\end{array} & \text { Lithology } \\ \text { 325CBNS } & 0 & 150 & \text { SHLE } \\ \text { 325CBNS } & 150 & 230 & \text { SDSL } \\ \text { 325KRBS } & 230 & 630 & \text { SDSL } \\ \text { 325KRBS } & 630 & 960 & \text { SHLE } \\ \text { 325KRBS } & 960 & 1,240 & \text { SDSL } \\ \text { 325KRBS } & 1,240 & 1,776 & \text { SHLE } \\ \text { 325CHRK } & 1,776 & 2,025 & \text { SDSL } \\ \text { 325CHRK } & 2,025 & 2,102 & \text { SHLE } \\ \text { 325HRSR } & 2,102 & 2,128 & \text { SNDS } \\ \text { 325HRSR } & 2,128 & 3,000 & \text { SHLE } \\ \text { 326ATCK } & 3,000 & 3,200 & \text { SDSL } \\ \text { 326ATCK } & 3,200 & 3,820 & \text { SHLE } \\ \text { 328WPCE } & 3,820 & 3,950 & \text { LMSN } \\ \text { 328WPCK } & 3,950 & 4,000 & \text { SHLE } \\ \text { 328WPCK } & 4,000 & 4,057 & \text { LMSH } \\ \text { 327UNVL } & 4,057 & 4,100 & \text { LMSH } \\ \text { 327UNVL } & 4,100 & 4,160 & \text { LMSH } \\ \text { 327UNVL } & 4,160 & 4,255 & \text { SNDS } \\ \text { 327UNVL } & 4,255 & 4,445 & \text { SDSL } \\ \text { 333DLCK } & 4,445 & 4,650 & \text { SHLE } \\ \text { 338OGKR } & 4,650 & 4,815 & \text { SHLE } \\ \text { 341WDFD } & 4,815 & 4,820 & \text { SDSL } \\ \text { 340HNTN } & 4,820 & 4,955 & \text { LMSN } \\ \text { 361SLVN } & 4,955 & 5,000 & \text { SHLE } \\ \text { 361VIOL } & 5,000 & 5,016 & \text { IMSN } \\ \text { 364SMPS } & 5,016 & 5,055 & \text { LMSN } \\ \text { 364SMPS } & 5,055 & 5,210 & \text { SNDS } \\ \text { 364SMPS } & 5,210 & 5,250 & \text { SHLE } \\ \text { 364SMPS } & 5,250 & 5,411 & \text { SNDS } \\ \text { 367ABCK } & 5,411 & & \text { DLMT } \\ & & & \end{array}$


Table 3.--Geologic logs for wells in the study area--Continued

Major County, Oklahoma

Latitude: 362139

Owner: SUPERIOR

Use of site: P

Altitude: 1,276
Long1tude: 981527

Local number: 22N-10W-27 BAA 1

Completion date: 01/01/1945

Depth of well: 8,279

\begin{tabular}{|c|c|c|c|}
\hline $\begin{array}{l}\text { Geologic- } \\
\text { unit code }\end{array}$ & $\underset{\text { Depth to }}{\text { top }}$ & $\begin{array}{l}\text { Depth to } \\
\text { bottom }\end{array}$ & Lithology \\
\hline $318 \mathrm{HNSS}$ & 0 & 200 & SLSN \\
\hline $318 \mathrm{HNSS}$ & 200 & 1,215 & SHLE \\
\hline 310WLNG & 1,215 & 1,583 & SHLE \\
\hline 310WLNG & 1,583 & 1,800 & ANDR \\
\hline 310WLNG & 1,800 & 2,100 & ANDR \\
\hline 310WLNG & 2,100 & 2,387 & ANDR \\
\hline 310NLNS & 2,387 & 2,458 & IMSH \\
\hline 317WFLD & 2,458 & 2,511 & SHLE \\
\hline $319 \mathrm{CHSE}$ & 2,511 & 2,542 & LMSH \\
\hline 317BRNS & 2,542 & 2.687 & LMSH \\
\hline $319 \mathrm{CHSE}$ & 2,687 & 2,762 & LMSH \\
\hline 317WRFD & 2,762 & 2,807 & IMSH \\
\hline $319 \mathrm{CCGV}$ & 2,807 & 2,862 & SDSI \\
\hline $319 \mathrm{CCGV}$ & 2,862 & 2.972 & IMSH \\
\hline $319 \mathrm{CCGV}$ & 2,972 & 3,056 & SHLE \\
\hline 319GRNL & 3,056 & 3,111 & LMSH \\
\hline $319 \mathrm{CCGV}$ & 3,111 & 3,178 & SHLE \\
\hline $319 \mathrm{CCGV}$ & 3,178 & 3,282 & LMSN \\
\hline $319 \mathrm{CCGV}$ & 3,282 & 3,660 & LMSH \\
\hline 322WBNS & 3,660 & 4,280 & LMSH \\
\hline 322TOPK & 4,280 & 4,308 & LMSH \\
\hline 322 SHWN & 4,308 & 4,637 & SHLE \\
\hline 322 SHWN & 4,637 & 4,920 & SDSL \\
\hline 322ORED & 4,920 & 5,068 & IMSH \\
\hline 322DGLS & 5,068 & 5,230 & SHLE \\
\hline 323LNSG & 5,230 & 5,395 & SNDS \\
\hline $323 \mathrm{KSSC}$ & 5,395 & 5,668 & SHLE \\
\hline 323MSSR & 5,668 & 5.673 & LMSN \\
\hline 323MSSR & 5,673 & 5,817 & SNDS \\
\hline $323 \mathrm{MSSR}$ & 5,817 & 5,920 & SHLE \\
\hline 323HGSR & 5,920 & 5,928 & IMSN \\
\hline 323MSSR & 5,928 & 6,066 & SHIE \\
\hline 323PLSN & 6,066 & 6,235 & SHLE \\
\hline 325MRMN & 6,235 & 6,483 & IMSH \\
\hline $325 \mathrm{CBNS}$ & 6,483 & 6,695 & SHLE \\
\hline 332CSTR & 6,695 & 6,983 & SHLE \\
\hline 333MRMC & 6,983 & 7,572 & IMSN \\
\hline 339RDRK & 7.572 & 7632 & SLSN \\
\hline
\end{tabular}


Table 3.--Geologic logs for wells in the study area--Continued

Major County, Oklahoma--Continued

Latitude: 362139

Owner: SUPERIOR

Use of site: $P$

Altitude: 1,276
, Long1tude: 981527

Local number: 22N-10W-27 BAA 1

Completion date: $01 / 01 / 1945$

Depth of well: 8,279

Geologicunit code

Depth to Depth to top bottom

Lithology

341WDFD

340HNTN

36I SLVN

7,632

7,686

SHLE

7,686

7,706

DLMT

361VIOL

7,706

7,820

7,820

SHLE

364BRMD

364BRMD

7,890

8,104

7,890

IMSN

LMSN

SNDS 
Table 3.--Geologic logs for wells in the study area--Continued

Mayes County, Oklahoma

Latitude: 360823

Owner: BALLOU

Use of site: $P$

Altitude:

\section{0}

Longitude: 951111

Local number: $19 \mathrm{~N}-20 \mathrm{E}-09$ BCD 1

Completion date: 01/01/1967

Depth of well: 1,124

$\begin{array}{lrcc}\begin{array}{l}\text { Geologic- } \\ \text { unit code }\end{array} & \begin{array}{r}\text { Depth to } \\ \text { top }\end{array} & \begin{array}{c}\text { Depth to } \\ \text { bottom }\end{array} & \text { Lithology } \\ \text { 364SMPS } & 175 & 227 & \text { SHLE } \\ \text { 367CTTR } & 227 & 497 & \text { DLMT } \\ \text { 368JFRC } & 497 & 695 & \text { DLMT } \\ \text { 367RBDX } & 695 & 790 & \text { DLMT } \\ \text { 367GSCD } & 790 & 1,102 & \text { DLMT } \\ \text { 400PCMB } & 1,102 & & \text { RYLT }\end{array}$

Mayes County, Oklahoma

Latitude: 362600

Owner: DAVIS

Use of site: T

Altitude:
670
Longitude: 950250

Local number: 23N-21E-34 ACA 1

Completion date: 01/01/1959

Depth of well: 1,622

$\begin{array}{lrrc}\begin{array}{l}\text { Geologic- } \\ \text { unit code }\end{array} & \begin{array}{r}\text { Depth to } \\ \text { top }\end{array} & \begin{array}{r}\text { Depth to } \\ \text { bottom }\end{array} & \text { Lithology } \\ \text { 368PWLL } & 170 & 320 & \text { DLMT } \\ \text { 367CTTR } & 320 & 700 & \text { DLMT } \\ \text { 368JFRC } & 700 & 940 & \text { DLMT } \\ \text { 367RBDX } & 940 & 1,080 & \text { DLMT } \\ \text { 367GSCD } & 1,080 & 1,320 & \text { DLMT } \\ \text { 367GNTR } & 1,320 & 1,340 & \text { SNDS } \\ \text { 371EMCP } & 1,340 & 1,450 & \text { DLMT } \\ \text { 371BNTR } & 1,450 & 1,615 & \text { DLMT } \\ \text { 400PCMB } & 1,615 & & \text { GRNT }\end{array}$


Table 3.--Geologic logs for wells in the study area--Continued

Murray County, Oklahoma

Latitude: 343046

Owner: FLEMING

Use of site:

Altitude:

995

Longitude: 970040

Local number: 01N-03E-32 CAA 1

Completion date: 06/04/1973

Depth of well: 5,178

\begin{tabular}{lrr|c}
$\begin{array}{l}\text { Geologic- } \\
\text { unit code }\end{array}$ & $\begin{array}{r}\text { Depth to } \\
\text { top }\end{array}$ & $\begin{array}{c}\text { Depth to } \\
\text { bottom }\end{array}$ & Lithology \\
320PSLV & 0 & 50 & SNDS \\
320PSLV & 50 & 510 & SHLE \\
320PSLV & 510 & 750 & LMSH \\
367ABCK & 750 & 1,050 & LMDM \\
367ABCK & 1,050 & 1,810 & DLMT \\
367ABCR & 1,810 & 2,550 & DLMT \\
367ABCR & 2,550 & 3,420 & DLMT \\
367ABCR & 3,420 & 4,700 & DLMT \\
367ABCR & 4,700 & 4,960 & LMSH \\
367ABCR & 4,960 & 5,000 & DLMT \\
367ABCR & 5,000 & 5,060 & SNDS \\
371REGN & 5,060 & 5,170 & SNDS \\
400PCMB & 5,170 & & GRNT
\end{tabular}

Muskogee County, Oklahoma

Latitude: 353510

Owner: MARSHALL

Use of site: $P$

Altitude: $\quad 601$
Longitude: 951528

Local number: $13 \mathrm{~N}-19 \mathrm{E}-23 \mathrm{DB} 1$

Completion date: $01 / 09 / 1958$

Depth of well: 3,296

\begin{tabular}{lrr|c}
$\begin{array}{l}\text { Geologic- } \\
\text { unit code }\end{array}$ & $\begin{array}{r}\text { Depth } \\
\text { top }\end{array}$ & $\begin{array}{c}\text { Depth to } \\
\text { bottom }\end{array}$ & Lithology \\
331PTKN & 280 & 340 & SNDS \\
326ATCK & 340 & 884 & SHLE \\
328WPCK & 884 & 1,039 & LMSN \\
331PTKN & 1,039 & 1,120 & LMSN \\
333DLCR & 1,120 & 1,258 & SHLE \\
338OGKR & 1,258 & 1,345 & LMSN \\
341WDFD & 1,345 & 1,370 & SHLE \\
340HNTN & 1,370 & 1,452 & LMSN \\
361SLVN & 1,452 & 1,500 & DLMT \\
361VIOL & 1,500 & 1,546 & LMSN \\
364SMPS & 1,546 & 1,548 & SNDS \\
364TYNR & 1,548 & 1,745 & SNDS \\
364BRGN & 1,745 & 1,840 & SNDS \\
367ABCR & 1,840 & 3,282 & LMSN \\
400PCMB & 3,282 & & GRNT
\end{tabular}


Table 3.--Geologic logs for wells in the study area--Continued

Noble County, Oklahoma

Latitude: 361218

Owner: HUBBART

Use of site: $P$

Altitude: 1,100
Longitude: 972036

Local number: 20N-01W-18 CDB I

Completion date:

Depth of well: 5,239

$\begin{array}{lrrc}\begin{array}{l}\text { Geologic- } \\ \text { un1t code }\end{array} & \begin{array}{r}\text { Depth } \\ \text { top }\end{array} & \begin{array}{c}\text { Depth to } \\ \text { bottom }\end{array} & \text { L1thology } \\ \text { 31OWLNG } & 0 & 760 & \text { SHLE } \\ \text { 317HRNG } & 760 & 785 & \text { DLMT } \\ \text { 319WFMP } & 785 & 1,350 & \text { SHLE } \\ \text { 319WFMP } & 1,350 & 1,790 & \text { SHLE } \\ \text { 322VRGL } & 1,790 & 2,720 & \text { SHLE } \\ \text { 322ORED } & 2,720 & 2,760 & \text { LMSN } \\ \text { 322VRGL } & 2,760 & 2,870 & \text { SHLE } \\ \text { 322VRGL } & 2,870 & 3,135 & \text { SDSL } \\ \text { 322VRGL } & 3,135 & 3,340 & \text { SDSL } \\ \text { 323LNSG } & 3,340 & 3,460 & \text { LMSN } \\ \text { 323MSSR } & 3,460 & 3,815 & \text { SHLE } \\ \text { 323MSSR } & 3,815 & 3,920 & \text { SNDS } \\ \text { 323MSSR } & 3,920 & 4,290 & \text { SDSL } \\ \text { 323MSSR } & 4,290 & 4,365 & \text { LMSH } \\ \text { 325MRMN } & 4,365 & 4,430 & \text { SHLE } \\ \text { 325MRMN } & 4,430 & 4,635 & \text { LMSN } \\ \text { 325CHRK } & 4,635 & 4,880 & \text { LMSN } \\ \text { 330MSSP } & 4,880 & 4,950 & \text { LMSN } \\ \text { 341WDFD } & 4,950 & 4,990 & \text { SHLE } \\ \text { 361SLVN } & 4,990 & 5,045 & \text { SHLE } \\ \text { 361VIOL } & 5,045 & 5,085 & \text { LMSN } \\ \text { 364BRMD } & 5,085 & 5,110 & \text { LMSN } \\ \text { 364SMPS } & 5,110 & 5,133 & \text { DLMT } \\ \text { 364SMPS } & 5,133 & & \text { SNDS }\end{array}$

Noble County, Oklahoma

Latitude: 361216

Owner: MAGNEY

Use of site: $P$

Altitude: 1,269
Longitude: 972626

Local number: 20N-02W-17 CC 1 Completion date:

Depth of well: 7,088

$\begin{array}{lrcc}\begin{array}{l}\text { Geologic- } \\ \text { unit code }\end{array} & \begin{array}{r}\text { Depth to } \\ \text { top }\end{array} & \begin{array}{c}\text { Depth to } \\ \text { bottom }\end{array} & \text { Lithology } \\ \text { 300PLZC } & 0 & 5,274 & \text { SDMN } \\ \text { 364SMPS } & 5,274 & 5,495 & \text { SDSL } \\ \text { 367ABCK } & 5,495 & 6,950 & \text { DLMT } \\ \text { 371REGN } & 6,950 & 7,050 & \text { SNDS } \\ \text { 400PCMB } & 7,050 & & \text { GRNT }\end{array}$


Table 3.--Geologic logs for wells in the study

area--Continued

Nowata County, Oklahoma

Latitude: 365100

Owner: ALSUP

Use of site: $P$

Altitude: $\quad 760$
Longitude: 953920

Local number: 27N-15E-01 DBB 1

Completion date: 06/07/1954

Depth of well: 2,222

\begin{tabular}{lrc|c}
$\begin{array}{l}\text { Geologic- } \\
\text { un1t code }\end{array}$ & $\begin{array}{r}\text { Depth to } \\
\text { top }\end{array}$ & $\begin{array}{c}\text { Depth to } \\
\text { bottom }\end{array}$ & Lithology \\
325LNPH & 101 & 132 & LMSN \\
325NOWT & 132 & 250 & SHLE \\
325ALMN & 250 & 282 & LMSN \\
325BNDR & 282 & 335 & SHLE \\
325PWNE & 335 & 369 & LMSN \\
325LBTT & 369 & 520 & SHLE \\
325FRSC & 520 & 595 & LMSN \\
325CHRK & 595 & 1,135 & SHLE \\
331BOON & 1,135 & 1,395 & LMSN \\
341CTNG & 1,395 & 1,465 & SHLE \\
367ABCK & 1,465 & & LMSN
\end{tabular}

Nowata County, Oklahoma

Latitude: 364712

Owner: PRAIRIE O G Use of site:

Altitude: $\quad 665$
Longitude: 953518

Local number: 27N-16E-27 CDC 1 Completion date:

Depth of well: 2,070

\begin{tabular}{lrr|c}
$\begin{array}{l}\text { Geologic- } \\
\text { unit code }\end{array}$ & $\begin{array}{r}\text { Depth to } \\
\text { top }\end{array}$ & $\begin{array}{c}\text { Depth to } \\
\text { bottom }\end{array}$ & L1thology \\
325PWNE & 0 & 30 & LMSN \\
325LBTT & 30 & 190 & SHLE \\
325FRSC & 190 & 260 & LMSN \\
325CHRK & 260 & 840 & SHLE \\
331BOON & 840 & 1,140 & LMSN \\
330CTNG & 1,140 & 1,200 & SHLE \\
367ABCK & 1,200 & 1,955 & LMSN \\
400PCMB & 1,955 & & GRNT
\end{tabular}


Table 3.--Geologic logs for wells in the study area--Continued

Okfuskee County, Oklahoma

Latitude: 353055

Owner: REPLOGLE

Use of site: P Altitude:
944
Longitude: 962714

Local number: 12N-08E-15 CAB 1 Completion date:

Depth of well: 4,507

$\begin{array}{lrcc}\begin{array}{c}\text { Geologic- } \\ \text { un1t code }\end{array} & \begin{array}{c}\text { Depth to } \\ \text { top }\end{array} & \begin{array}{c}\text { Depth to } \\ \text { bottom }\end{array} & \text { Lithology } \\ \text { 323MSSR } & 0 & 1,020 & \text { SDSL } \\ \text { 323MSSR } & 1,020 & 1,080 & \text { SNDS } \\ \text { 323MSSR } & 1,080 & 1,330 & \text { SHLE } \\ \text { 323CCKB } & 1,330 & 1,350 & \text { LMSN } \\ \text { 325MRMN } & 1,350 & 1,400 & \text { LMSH } \\ \text { 325MRMN } & 1,400 & 1,630 & \text { SHLE } \\ \text { 325MRMN } & 1,630 & 2,080 & \text { SDSL } \\ \text { 325MRMN } & 2,080 & 2,400 & \text { SHLE } \\ \text { 325CHRK } & 2,400 & 2,500 & \text { SNDS } \\ \text { 325CHRR } & 2,500 & 2,700 & \text { SHLE } \\ \text { 325CHRR } & 2,700 & 2,810 & \text { SNDS } \\ \text { 325CHRR } & 2,810 & 2,900 & \text { SHLE } \\ \text { 325CHRR } & 2,900 & 3,050 & \text { SDSL } \\ \text { 325CHRK } & 3,050 & 3,160 & \text { SHLE } \\ \text { 325CHRR } & 3,160 & 3,200 & \text { LMSH } \\ \text { 325CHRR } & 3,200 & 3,280 & \text { SDSL } \\ \text { 325CHRR } & 3,280 & 3,380 & \text { SHLE } \\ \text { 325CHRK } & 3,380 & 3,410 & \text { LMSH } \\ \text { 325CHRR } & 3,410 & 3,735 & \text { SHLE } \\ \text { 327UNVL } & 3,735 & 3,825 & \text { LMSH } \\ \text { 33OMSSP } & 3,825 & 3,850 & \text { LMSN } \\ \text { 33OMSSP } & 3,850 & 4,235 & \text { SHLE } \\ \text { 341WDFD } & 4,235 & 4,263 & \text { SHLE } \\ \text { 34OMSNR } & 4,263 & 4,264 & \text { SNDS } \\ \text { 34OHNTN } & 4,264 & 4,328 & \text { DLMT } \\ \text { 361SLVN } & 4,328 & 4,415 & \text { SHLE } \\ \text { 361VIOL } & 4,415 & 4,435 & \text { DLMT } \\ \text { 364BRMD } & 4,435 & & \text { DLMT }\end{array}$


Table 3.--Geologic logs for wells in the study area-Continued

Oklahoma County, Oklahoma

Latitude: 352505

Owner: FARLEY

Use of site: $P$

Alt1tude: 1,242
Longitude: 972712

Local number: 11N-02W-19 BAC 1

Completion date :

Depth of well: 8,342

\begin{tabular}{lrc|c}
$\begin{array}{l}\text { Geologic- } \\
\text { un1t code }\end{array}$ & $\begin{array}{r}\text { Depth } \\
\text { top }\end{array}$ & $\begin{array}{c}\text { Depth to } \\
\text { bottom }\end{array}$ & Lithology \\
318LNRD & 0 & 1,000 & SDSL \\
319WFMP & 1,000 & 2,910 & SDSL \\
322VRGL & 2,910 & 2,950 & LMSN \\
322VRGL & 2,950 & 3,170 & SHLE \\
322SHWN & 3,170 & 3,400 & LMSD \\
322SHWN & 3,400 & 3,490 & SHLE \\
322SHWN & 3,490 & 3,780 & SNDS \\
322SHWN & 3,780 & 3,950 & SHLE \\
322SHWN & 3,950 & 4,020 & SNDS \\
322SHWN & 4,020 & 4,155 & SDSL \\
322ORED & 4,155 & 4,200 & LMSN \\
322DGLS & 4,200 & 4,340 & SDSL \\
322DGLS & 4,340 & 4,450 & LMSH \\
322DGLS & 4,450 & 4,600 & SNDS \\
323MSSR & 4,600 & 4,840 & SHLE \\
323MSSR & 4,840 & 4,870 & LMSN \\
323MSSR & 4,870 & 4,990 & SDSL \\
323MSSR & 4,990 & 5,090 & SHLE \\
323HGSR & 5,090 & 5,220 & LMSN \\
323MSSR & 5,220 & 5,470 & SHLE \\
323CGKB & 5,470 & 5,490 & LMSN \\
325MRMN & 5,490 & 5,890 & SHLE \\
325MRMN & 5,890 & 5,970 & LMSH \\
325MRMN & 5,970 & 6,010 & SDSL \\
367ABCK & 6,010 & 8,240 & DLMT \\
371REGN & 8,240 & 8,260 & SNDS \\
400PCMB & 8,260 & & GRNT \\
& & &
\end{tabular}


Table 3.--Geologic logs for wells in the study area--Continued

Oklahoma County, Oklahoma

Latitude: 353755

Owner: MACKLIN Use of site: P

Altitude: 1,030
Longitude: 971525

Local number: $13 \mathrm{~N}-01 \mathrm{~W}-01$ BDD 1 Completion date:

Depth of well: 5,942

\begin{abstract}
Geologicunit code

318LNRD

319WFMP

322VRGL

322VRGL

322VRGL

322ORED

323MSSR

323MSSR

323MSSR

323HGSR

323MSSR

323CCKB

325MRMN

325MRMN

325MRMN

34 IWDFD

34OMSNR

340HNTN

36ISLVN

36IVIOL

364SMPS

364SMPS
\end{abstract}

Depth to Depth to bottom

540

2,315

2,420

2,740

3,835

3,890

4,280

4,445

4,560

4,595

4,835

4,845

5,070

5,170

5,500

5,550

5,555

5,617

5,720

5,755

5,810
Lithology

SDSL

SHLE

IMSH

SHLE

SHLE

LMSN

SHLE

LMSH

SHLE

LMSH

SHLE

LMSN

SHLE

LMSH

SHLE

SHLE

SNDS

IMSN

SHLE

IMSN

DLMT

SDSL 
Table 3.--Geologic logs for wells in the study area--Continued

Okmulgee County, Oklahoma

Latitude: 354922

Owner : MILLER

Use of site: $P$ Altitude:
802
Longitude: 955050

Local number: 16N-14E-32 BDA 1 Completion date:

Depth of well: 3,915
Geologic- Depth to Depth to unit code top bottom

325 WWOK

325CHRK

325CHRK

325CHRK

326ATCK

332CSTR

33OMSSP

341CTNG

364 SMPS

367ABCK

0
100
450
615
1,455
1,840
2,050
2,250
2,272
2,608

100

450

615

1,455

1,840

2,250

2,250

2, 272

2,608

Lithology

SHLE

SDSL

SLSN

SDSL

SHLE

SHLE

IMSH

SHLE

SDSL

DLMT 
Table 3.--Geologic logs for wells in the study area--Continued

Osage County, Oklahoma

Latitude: 361940

Owner: ZINK

Use of site: P

Altitude :
890
Longitude: 960915

Local number: 21N-11E-05 BC 1 Completion date:

Depth of well: 2,995

$\begin{array}{lrrc}\begin{array}{l}\text { Geologic- } \\ \text { unit code }\end{array} & \begin{array}{r}\text { Depth to } \\ \text { top }\end{array} & \begin{array}{r}\text { Depth to } \\ \text { bottom }\end{array} & \text { Lithology } \\ \text { 323WANN } & 0 & 190 & \text { SDSL } \\ \text { 323MSSR } & 190 & 1,056 & \text { SDSL } \\ \text { 323MSSR } & 1,056 & 1,186 & \text { SNDS } \\ \text { 325MRMN } & 1,186 & 1,356 & \text { SHLE } \\ \text { 325MRMN } & 1,356 & 1,408 & \text { LMSN } \\ \text { 325CHRK } & 1,408 & 1,762 & \text { SHLE } \\ \text { 325CHRK } & 1,762 & 1,852 & \text { SNDS } \\ \text { 325CHRK } & 1,852 & 1,940 & \text { SHLE } \\ \text { 330MSSP } & 1,940 & 2,130 & \text { LMSN } \\ \text { 341CTNG } & 2,130 & 2,136 & \text { SHLE } \\ \text { 364SMPS } & 2,136 & 2,228 & \text { SNDS } \\ \text { 364SMPS } & 2,228 & 2,298 & \text { SHLE } \\ \text { 367ABCK } & 2,298 & 2,922 & \text { DLMT } \\ \text { 400PCMB } & 2,922 & & \text { GRNT }\end{array}$

Osage County, Oklahoma

Latitude: 362015

Owner: SUNRAY

Use of site: $P$

Altitude: 836
Longitude: 961313

Local number: 22N-10E-34 C 1 Completion date:

Depth of well: 3,235

$\begin{array}{lrrc}\begin{array}{l}\text { Geologic- } \\ \text { unit code }\end{array} & \begin{array}{r}\text { Depth to } \\ \text { top }\end{array} & \begin{array}{r}\text { Depth to } \\ \text { bottom }\end{array} & \text { Lithology } \\ \text { 323BRDL } & 0 & 40 & \text { SHLE } \\ \text { 323MSSR } & 40 & 1,225 & \text { SHLE } \\ \text { 323MSSR } & 1,225 & 1,306 & \text { SNDS } \\ \text { 325MRMN } & 1,306 & 1,470 & \text { SHLE } \\ \text { 325MRMN } & 1,470 & 1,560 & \text { LMSN } \\ \text { 325CHRR } & 1,560 & 1,914 & \text { SHLE } \\ \text { 325CHRR } & 1,914 & 1,990 & \text { SNDS } \\ \text { 325CHRR } & 1,990 & 2,055 & \text { SHLE } \\ \text { 330MSSP } & 2,055 & 2,354 & \text { LMSN } \\ \text { 341CTNG } & 2,354 & 2,358 & \text { SHLE } \\ \text { 364SMPS } & 2,358 & 2,420 & \text { SDSL } \\ \text { 367ABCR } & 2,420 & & \text { DLMT }\end{array}$


Table 3.--Geologic logs for wells in the study area--Continued

Osage County, Oklahoma

Latitude: 363722

Owner: PHILLIPS

Use of site: P

Altitude: $\quad 898$
Long1tude: 962512

Local number: 25N-08E-23 CCC 1

Completion date: 08/15/1956

Depth of well: 3,663

$\begin{array}{lrrr}\begin{array}{l}\text { Geologic- } \\ \text { unit code }\end{array} & \begin{array}{r}\text { Depth } \\ \text { top }\end{array} & \begin{array}{r}\text { Depth to } \\ \text { bottom }\end{array} & \text { Lithology } \\ \text { 322VMOS } & 0 & 688 & \text { SDSL } \\ \text { 323MSSR } & 688 & 1,144 & \text { SHLE } \\ \text { 323MSSR } & 1,144 & 1,440 & \text { SNDS } \\ \text { 323MSSR } & 1,440 & 1,710 & \text { SHLE } \\ \text { 325MRMN } & 1,710 & 1,958 & \text { SHLE } \\ \text { 325CHRK } & 1,958 & 2,296 & \text { SHLE } \\ \text { 330MSSP } & 2,296 & 2,625 & \text { LMSN } \\ \text { 341CTNG } & 2,625 & 2,645 & \text { SHLE } \\ \text { 367ABCR } & 2,645 & 3,660 & \text { DLMT } \\ \text { 400PCMB } & 3,660 & & \text { GRNT }\end{array}$

Ottawa County, Oklahoma

Latitude: 365324

Owner: GOODRICH RUB

Use of site: W

Altitude: $\quad 798$
Long1tude: 945313

Local number: 28N-22E-24 CAD 1 Completion date: 01/01/1944

Depth of well: 1,055

\begin{tabular}{lrr|c}
$\begin{array}{l}\text { Geologic- } \\
\text { unit code }\end{array}$ & $\begin{array}{r}\text { Depth to } \\
\text { top }\end{array}$ & $\begin{array}{r}\text { Depth to } \\
\text { bottom }\end{array}$ & Lithology \\
332CSTR & 22 & 45 & LMSN \\
333WRSW & 45 & 167 & LMSN \\
338SRCR & 167 & 175 & LMSN \\
3380SGE & 175 & 255 & LMSN \\
3380SGE & 255 & 435 & CHRT \\
341CTNG & 435 & 443 & SHLE \\
367CTTR & 443 & 560 & DLMT \\
368SCRR & 560 & 585 & DLMT \\
368JFRC & 585 & 890 & DLMT \\
367RBDX & 890 & 1,045 & DLMT \\
40OPCMB & 1,045 & & GRNT
\end{tabular}


Table 3.--Geologic logs for wells in the study area-Continued

Ottawa County, Oklahoma

Latitude: 365213

Owner: MIAMI, OK

Use of site: W Altitude:
Longitude: 945007

Local number: 28N-23E-33 BA 1 Completion date: 01/01/1955

Depth of well: 1,526

$\begin{array}{lrrc}\begin{array}{l}\text { Geologic- } \\ \text { un1t code }\end{array} & \begin{array}{r}\text { Depth to } \\ \text { top }\end{array} & \begin{array}{c}\text { Depth to } \\ \text { bottom }\end{array} & \text { Lithology } \\ \text { 333WRSW } & 0 & 130 & \text { LMSN } \\ \text { 338SRCK } & 130 & 150 & \text { LMSN } \\ \text { 338OSGE } & 150 & 250 & \text { LMSN } \\ \text { 338OSGE } & 250 & 360 & \text { LMSN } \\ \text { 338FRGL } & 360 & 405 & \text { LMSN } \\ \text { 339NRTV } & 405 & 428 & \text { SHLE } \\ \text { 341CTNG } & 428 & 440 & \text { SHLE } \\ \text { 367CTTR } & 440 & 675 & \text { DLMT } \\ \text { 368JFRC } & 675 & 906 & \text { DLMT } \\ \text { 367RBDX } & 906 & 1,070 & \text { DLMT } \\ \text { 367GSCDU } & 1,070 & 1,135 & \text { DLMT } \\ \text { 367GSCDL } & 1,135 & 1,350 & \text { DLMT } \\ \text { 367GNTR } & 1,350 & 1,370 & \text { SNDS } \\ \text { 371EMNC } & 1,370 & 1,485 & \text { DLMT } \\ \text { 371DRBD } & 1,485 & & \text { DLMT }\end{array}$


Table 3.--Geologic logs for wells in the study area--Continued

Pawnee County, Oklahoma

Latitude: 362551

Owner: TRIGG Use of site: P Altitude : 959

Longitude: 965836

Local number: 23N-03E-34 BCB 1 Completion date:

Depth of well: 4,586

\begin{tabular}{lrr|r}
$\begin{array}{l}\text { Geologic- } \\
\text { unit code }\end{array}$ & $\begin{array}{r}\text { Depth to } \\
\text { top }\end{array}$ & $\begin{array}{c}\text { Depth to } \\
\text { bottom }\end{array}$ & Lithology \\
3180SCR & 0 & 300 & SHLE \\
322VNSS & 300 & 800 & LMSH \\
322ADA & 800 & 1,200 & SHLE \\
322VMOS & 1,200 & 1,700 & SDSL \\
322DGLS & 1,700 & 1,820 & SDSL \\
323MSSR & 1,820 & 2,270 & SDSL \\
323MSSR & 2,270 & 2,310 & LMSN \\
323MSSR & 2,310 & 2,440 & SDSL \\
323MSSR & 2,440 & 2,720 & SHLE \\
323MSSR & 2,720 & 2,825 & SDSL \\
323MSSR & 2,825 & 3,155 & SDSL \\
323CCKB & 3,155 & 3,170 & LMSN \\
323KSSC & 3,170 & 3,200 & SDSL \\
325MRMN & 3,200 & 3,295 & LMSH \\
325MRMN & 3,295 & 3,490 & IMSN \\
325CHRK & 3,490 & 3,815 & SHLE \\
330MSSP & 3,815 & 4,070 & LMSN \\
341WDFD & 4,070 & 4,110 & SHLE \\
340MSNR & 4,110 & 4,112 & SNDS \\
364SMPS & 4,112 & 4,120 & DLMT \\
364SMPS & 4,120 & 4,130 & SNDS \\
364SMPS & 4,130 & 4,335 & SDSL \\
367ABCK & 4,335 & & DLMT \\
& & &
\end{tabular}


Table 3.--Geologic logs for wells in the study area--Continued

Payne County, Oklahoma

Latitude: 361024

Owner: FLYNN

Use of site: $P$ Altitude :
960
Longitude: 970614

Local number: 2ON-02E-33 BBB 1 Completion date:

Depth of well: 4,877

$\begin{array}{lrcc}\begin{array}{l}\text { Geologic- } \\ \text { un1t oode }\end{array} & \begin{array}{r}\text { Depth to } \\ \text { top }\end{array} & \begin{array}{c}\text { Depth to } \\ \text { bottom }\end{array} & \text { L1thology } \\ \text { 310WLNG } & 0 & 200 & \text { SDSL } \\ \text { 318OSCR } & 200 & 600 & \text { SDSL } \\ \text { 322VNSS } & 600 & 760 & \text { SDSL } \\ \text { 322VNSS } & 760 & 1,150 & \text { SHLE } \\ \text { 322ADA } & 1,150 & 1,370 & \text { LMSH } \\ \text { 322ADA } & 1,370 & 1,620 & \text { SHLE } \\ \text { 322VMOS } & 1,620 & 1,850 & \text { SDSL } \\ \text { 322VMOS } & 1,850 & 2,240 & \text { SHLE } \\ \text { 322VMOS } & 2,240 & 2,310 & \text { SNDS } \\ \text { 323MSSR } & 2,310 & 2,850 & \text { SDSL } \\ \text { 323MSSR } & 2,850 & 2,900 & \text { LMSH } \\ \text { 323MSSR } & 2,900 & 3,100 & \text { SHLE } \\ \text { 323MSSR } & 3,100 & 3,200 & \text { SDSL } \\ \text { 323MSSR } & 3,200 & 3,640 & \text { SHLE } \\ \text { 323CCKB } & 3,640 & 3,650 & \text { LMSN } \\ \text { 323MSSR } & 3,650 & 3,840 & \text { SHLE } \\ \text { 325MRMN } & 3,840 & 4,030 & \text { IMSH } \\ \text { 325CHRK } & 4,030 & 4,110 & \text { LMSH } \\ \text { 325CHRK } & 4,110 & 4,120 & \text { LMSN } \\ \text { 325CHRK } & 4,120 & 4,210 & \text { SHLE } \\ \text { 325CHRK } & 4,210 & 4,230 & \text { LMSN } \\ \text { 325CHRK } & 4,230 & 4,260 & \text { SHLE } \\ \text { 325CHRK } & 4,260 & 4,280 & \text { SNDS } \\ \text { 325CHRK } & 4,280 & 4,430 & \text { SHLE } \\ \text { 330MSSP } & 4,430 & 4,640 & \text { LMSN } \\ \text { 341WDFD } & 4,640 & 4,680 & \text { SHLE } \\ \text { 361SLVN } & 4,680 & 4,740 & \text { SHLE } \\ \text { 361VIOL } & 4,740 & 4,780 & \text { LMSN } \\ \text { 364BRMD } & 4,780 & 4,805 & \text { LMSN } \\ \text { 364BRMD } & 4,805 & 4,820 & \text { DLMT } \\ \text { 364SMPS } & 4,820 & 4,850 & \text { SNDS } \\ \text { 364SMPS } & 4,850 & 4,860 & \text { DLMT } \\ \text { 364SMPS } & 4,860 & & \text { SNDS }\end{array}$


Table 3.--Geologic logs for wells in the study area--Continued

Pittsburg County, Oklahome

Lat1tude: 345558

Owner: SEALY

Use of site: $P$

Altitude :
796
Long1tude: 955923

Local number: 05N-12E-01 CA 1

Completion date: 06/01/1964

Depth of well: 3,541

$\begin{array}{lrcc}\begin{array}{c}\text { Geologic- } \\ \text { un1t code }\end{array} & \begin{array}{r}\text { Depth to } \\ \text { top }\end{array} & \begin{array}{c}\text { Depth to } \\ \text { bottom }\end{array} & \text { Lithology } \\ \text { 325BGGY } & 0 & 1,460 & \text { SDSL } \\ \text { 325CHRR } & 1,460 & 2,112 & \text { SDSL } \\ \text { 325SVNN } & 2,112 & 2,855 & \text { IMSH } \\ \text { 325CHRR } & 2,855 & 3,320 & \text { SDSL } \\ \text { 325HRSR } & 3,320 & 3,410 & \text { SNDS } \\ \text { 326ATOR } & 3,410 & & \text { SHLE }\end{array}$

P1ttsburg County, Oklahoma

Latitude: 345758

Owner: MANSHRICK

Use of site:

Altitude :

963
Long1tude: 953023

Local number: 06N-17E-28 ADB 1 Completion date: 09/23/1954

Depth of well: 12,915

\begin{tabular}{lrr|c}
$\begin{array}{l}\text { Geologic- } \\
\text { un1t code }\end{array}$ & $\begin{array}{r}\text { Depth to } \\
\text { top }\end{array}$ & $\begin{array}{r}\text { Depth to } \\
\text { bottom }\end{array}$ & L1thology \\
326ATCK & 3,325 & 8,970 & SHLE \\
326ATCK & 8,970 & 9,070 & SDSL \\
326ATCK & 9,070 & 9,895 & SHLE \\
328WPCK & 9,895 & 10,065 & LMSN \\
328WPCK & 10,065 & 10,500 & SHLE \\
327UNVL & 10,500 & 10,700 & SDSL \\
327UNVL & 10,700 & 10,895 & SLSH \\
333DLCK & 10,895 & 11,280 & LMSH \\
338OGKK & 11,280 & 11,410 & LMSN \\
341WDFD & 11,410 & 11,485 & SHLE \\
340MSNR & 11,485 & 11,495 & CHRT \\
361SLVN & 11,495 & 11,552 & SHLE \\
361FRVL & 11,552 & 11,630 & LMSN \\
364BRMD & 11,630 & 12,070 & DLMT \\
364BRMD & 12,070 & 12,330 & SNDS \\
367ABCK & 12,330 & & DLMT \\
367ABCK & 12,330 & & DLMT
\end{tabular}


Table 3.--Geologic logs for wells in the study area--Continued

Pontotoc County, Oklahoma

Lat1tude: 344057

Owner: BROWN

Use of site: $P$

Altitude: 1,177
Long1tude: 964806

Local number: 03N-05E-32 CD

Completion date: 03/24/1966

Depth of well: 5,741

$\begin{array}{lrrc}\begin{array}{l}\text { Geologic- } \\ \text { unit code }\end{array} & \begin{array}{r}\text { Depth to } \\ \text { top }\end{array} & \begin{array}{c}\text { Depth to } \\ \text { bottom }\end{array} & \text { Lithology } \\ \text { 322ADA } & 20 & 346 & \text { CGLM } \\ \text { 361SLVN } & 345 & 510 & \text { SHLE } \\ \text { 361VIOL } & 510 & 910 & \text { LMSN } \\ \text { 364SMPS } & 910 & 1,200 & \text { LMSH } \\ \text { 364SMPS } & 1,200 & 1,310 & \text { DLMT } \\ \text { 364SMPS } & 1,310 & 1,375 & \text { LMSH } \\ \text { 364SMPS } & 1,375 & 1,500 & \text { DLMT } \\ \text { 364SMPS } & 1,500 & 1,600 & \text { SNDS } \\ \text { 364SMPS } & 1,600 & 1,735 & \text { LMSH } \\ \text { 364SMPS } & 1,735 & 1,800 & \text { SNDS } \\ \text { 364SMPS } & 1,800 & 1,970 & \text { DMSH } \\ \text { 364SMPS } & 1,970 & 2,010 & \text { SNDS } \\ \text { 367ABCK } & 2,010 & 2,600 & \text { DLMT } \\ \text { 367ABCK } & 2,600 & 2,910 & \text { DLMT } \\ \text { 367ABCK } & 2,910 & 3,610 & \text { DLMT } \\ \text { 367ABCK } & 3,610 & 4,940 & \text { DLMT } \\ \text { 367ABCK } & 4,940 & 5,600 & \text { DLMT } \\ \text { 371REGN } & 5,600 & 5,720 & \text { SNDS } \\ \text { 400PCMB } & 5,720 & & \text { GRNT }\end{array}$


Table 3.--Geologic logs for wells in the study area--Continued

Pottawatomie County, Oklahoma

Latitude: 352108

Owner: EAVES

Use of site: $P$

Altitude: 1,078
Longitude: 970429

Local number: 10N-02E-10 DAC 1

Completion date:

Depth of well: 6,070

\begin{tabular}{lrr|l} 
Geologic- & $\begin{array}{r}\text { Depth } \\
\text { unit code }\end{array}$ & $\begin{array}{c}\text { Depth to } \\
\text { bottom }\end{array}$ & Lithology \\
310WLNG & 0 & 415 & SDSL \\
319WFMP & 415 & 1,900 & SDSL \\
322VRGL & 1,900 & 2,065 & SHLE \\
322SHWN & 2,065 & 2,265 & LMSH \\
322VRGL & 2,265 & 2,500 & SDSL \\
322VRGL & 2,500 & 3,250 & SHLE \\
322DGLS & 3,250 & 3,345 & SNDS \\
323MSSR & 3,345 & 3,485 & SHLE \\
324BLCT & 3,485 & 3,545 & SDSL \\
323MSSR & 3,545 & 3,735 & LMSH \\
323MSSR & 3,735 & 3,940 & SDSL \\
323HGSR & 3,940 & 3,980 & LMSH \\
323MSSR & 3,980 & 4,145 & SHLE \\
323CCKB & 4,145 & 4,195 & LMSN \\
323MSSR & 4,195 & 4,365 & SDSL \\
325MRMN & 4,365 & 4,555 & SDSL \\
325MRMN & 4,555 & 4,560 & LMSN \\
325MRMN & 4,560 & 4,960 & SHLE \\
325MRMN & 4,960 & 4,995 & LMSH \\
325CHRK & 4,995 & 5,070 & SDSL \\
325CHRK & 5,070 & 5,215 & SDSL \\
325CHRK & 5,215 & 5,330 & SDSL \\
325CHRK & 5,330 & 5,385 & LMSH \\
33OMSSP & 5,385 & 5,550 & LMSH \\
341WDFD & 5,550 & 5,700 & SHLE \\
34OHNTN & 5,700 & 5,710 & LMSN \\
361SLVN & 5,710 & 5,805 & SHLE \\
361VIOL & 5,805 & 5,885 & LMSN \\
364SMPS & 5,885 & 5,920 & LMSN \\
364SMPS & 5,920 & 5,980 & DLMT \\
364SMPS & 5,980 & & SNDS \\
& & &
\end{tabular}


Table 3.--Geologic logs for wells in the study area-Continued

Roger Mills County, Oklahoma

Lat1tude: 353101

Owner: GULF OIL

Use of site: $P$

Altitude: $\quad 2,070$
Longitude: 993542

Local number: $12 \mathrm{~N}-23 \mathrm{~W}-14$ AD 1

Completion date: 10/09/1951

Depth of well: 13,010

$\begin{array}{lrcc}\begin{array}{l}\text { Geologic- } \\ \text { unit code }\end{array} & \begin{array}{r}\text { Depth } \\ \text { top }\end{array} & \begin{array}{c}\text { Depth to } \\ \text { bottom }\end{array} & \text { L1thology } \\ \text { 313ELRN } & 400 & 750 & \text { SDSL } \\ \text { 313ELRN } & 750 & 900 & \text { SHLE } \\ \text { 313BLIN } & 900 & 1,070 & \text { ANDR } \\ \text { 313ELRN } & 1,070 & 1,200 & \text { SHLE } \\ \text { 313ELRN } & 1,200 & 1,280 & \text { SHLE } \\ \text { 313ELRN } & 1,280 & 2,110 & \text { SHLE } \\ \text { 313ELRN } & 2,110 & 2,470 & \text { SHLE } \\ \text { 31OPRMN } & 2,470 & 3,030 & \text { SHLE } \\ \text { 31OPRMN } & 3,030 & 3,205 & \text { SHLE } \\ \text { 31OWLNG } & 3,205 & 3,930 & \text { SHLE } \\ \text { 319CHSE } & 3,930 & 4,175 & \text { SHLE } \\ \text { 319CHSE } & 4,175 & 4,580 & \text { DLMT } \\ \text { 319CHSE } & 4,580 & 4,995 & \text { LMSN } \\ \text { 319CHSE } & 4,995 & 6,000 & \text { LMSH } \\ \text { 322VRGL } & 6,000 & 6,060 & \text { SNDS } \\ \text { 322VRGL } & 6,060 & 6,122 & \text { LMSN } \\ \text { 322VRGL } & 6,122 & 6,640 & \text { LMSH } \\ \text { 322VRGL } & 6,640 & 6,730 & \text { LMSN } \\ \text { 322VRGL } & 6,730 & 7,190 & \text { LMSH } \\ \text { 322VRGL } & 7,190 & 7,355 & \text { SHLE } \\ \text { 322VRGL } & 7,355 & 7,400 & \text { LMSH } \\ \text { 322VRGL } & 7,400 & 7,770 & \text { SNDS } \\ \text { 322VRGL } & 7,770 & 8,595 & \text { SDSL } \\ \text { 322DGLS } & 8,595 & 8,685 & \text { SNDS } \\ \text { 322DGLS } & 8,685 & 9,095 & \text { LMSH } \\ \text { 323LNSG } & 9,095 & 9,660 & \text { LMSH } \\ \text { 323HGSR } & 9,660 & 10,090 & \text { SDSL } \\ \text { 323CCEB } & 10,090 & 10,190 & \text { SHLE } \\ \text { 323CFVL } & 10,190 & 10,315 & \text { SDSL } \\ \text { 323CFVL } & 10,315 & 10,595 & \text { SDSL } \\ \text { 325DSMS } & 10,595 & 11,230 & \text { SHLE } \\ \text { 325CHRK } & 11,230 & 12,420 & \text { SHLE } \\ \text { 325CHRE } & 12,420 & & \text { SDSL } \\ & & & \\ & & & \end{array}$


Table 3.--Geologic logs for wells in the study area--Continued

Roger Mills County, Oklahoma

Latitude: 354737

Owner: CLARK CNDN

Use of site: $P$

Altitude: $\quad 2,140$
Longitude : 993343

Local number: 15N-22W-08 BDA 1

Completion date: 05/17/1972

Depth of well: 19,435

\begin{tabular}{|c|c|c|c|}
\hline $\begin{array}{l}\text { Geologic- } \\
\text { unit code }\end{array}$ & $\underset{\text { top }}{\text { Depth to }}$ & $\begin{array}{l}\text { Depth to } \\
\text { bottom }\end{array}$ & Lithology \\
\hline 319CHSE & 4,239 & 4,510 & LMSH \\
\hline 317FRRL & 4,510 & 4,690 & LMSN \\
\hline 319PNTC & 4,690 & 4,980 & SHLE \\
\hline 319PNTC & 4,980 & 5,250 & LMSN \\
\hline 319PNTC & 5,250 & 5,395 & SHLE \\
\hline 319PNTC & 5,395 & 5,660 & LMSH \\
\hline 319 PNTC & 5,660 & 5,800 & LMSN \\
\hline 319PNTC & 5,800 & 5,930 & SHLE \\
\hline 322VRGL & 5,930 & 6,089 & LMSN \\
\hline 322VRGL & 6.089 & 6,350 & SHLE \\
\hline 322VRGL & 6,350 & 6,480 & SNDS \\
\hline 322VRGL & 6,480 & 7,760 & SHLE \\
\hline 322VRGL & 7,760 & 7,880 & SHLE \\
\hline 322VRGL & 7,880 & 7,940 & SNDS \\
\hline 322VRGL & 7,940 & 8,250 & SHLE \\
\hline 322VRGL & 8,250 & 8,610 & SHLE \\
\hline 322DGLS & 8,610 & 8,710 & SNDS \\
\hline $323 \mathrm{HXBR}$ & 8,710 & 9,160 & SHLE \\
\hline 323LNSG & 9,160 & 9,210 & SHLE \\
\hline $323 \mathrm{HXBR}$ & 9,210 & 9.610 & SHLE \\
\hline 323HGSR & 9,610 & 9,660 & SHLE \\
\hline $323 \mathrm{HXBR}$ & 9,660 & 10,000 & SHLE \\
\hline $323 \mathrm{CCKB}$ & 10,000 & 10,110 & SHLE \\
\hline 323CFVI & 10,110 & 10,450 & SDSL \\
\hline 323CFVI & 10,450 & 10,550 & SHLE \\
\hline 325DSMS & 10,550 & 11,110 & SHLE \\
\hline 325CHRK & 11,110 & 11,170 & SHLE \\
\hline 325CHRK & 11,170 & 11,290 & LMSN \\
\hline $325 \mathrm{CHRK}$ & 11,290 & 11,520 & SHLE \\
\hline 325CHRK & 11,520 & 11,700 & IMSH \\
\hline $325 \mathrm{CHRK}$ & 11,700 & 12,100 & SHLE \\
\hline 325CHRK & 12,100 & 12,250 & SNDS \\
\hline 325CHRK & 12,250 & 13,080 & SHLE \\
\hline 325CHRK & 13,080 & 13,160 & SHLE \\
\hline 328MRRW & 13,160 & 13,990 & SHLE \\
\hline 328MRRW & 13,990 & 14,050 & IMSN \\
\hline 328MRRW & 14,050 & 14,430 & SHLE \\
\hline 328MRRW & 14,430 & 14,790 & SHLE \\
\hline
\end{tabular}


Table 3.--Geologic logs for wells in the study area--Continued

Roger Mills County, Oklahoma--Continued

Latitude: 354737

Owner: CLARK CNDN Use of site: $P$

Altitude: $\quad 2,140$
Longitude: 993343

Local number: 15N-22W-08 BDA 1 Completion date: 05/17/1972

Depth of well: 19,435

$\begin{array}{lrcc}\begin{array}{l}\text { Geologio- } \\ \text { unit oode }\end{array} & \begin{array}{r}\text { Depth to } \\ \text { top }\end{array} & \begin{array}{c}\text { Depth to } \\ \text { bottom }\end{array} & \text { L1thology } \\ \text { 328MRRW } & 14,790 & 14,960 & \text { SNDS } \\ \text { 328MRRW } & 14,960 & 15,260 & \text { SNDS } \\ \text { 332CSTR } & 15,260 & 15,520 & \text { IMSN } \\ \text { 332CSTR } & 15,520 & 15,970 & \text { SHLE } \\ \text { 332CSTR } & 15,970 & 16,180 & \text { SHLE } \\ \text { 332CSTR } & 16,180 & 16,410 & \text { LMSN } \\ \text { 332CSTR } & 16,410 & 16,880 & \text { LMSN } \\ \text { 332CSTR } & 16,880 & 17,030 & \text { SHLE } \\ \text { 332CSTR } & 17,030 & 17,140 & \text { LMSN } \\ \text { 330MSSP } & 17,140 & 17,870 & \text { LMSN } \\ \text { 330MSSP } & 17,870 & 18,710 & \text { IMSN } \\ \text { 341WDFD } & 18,710 & 18,830 & \text { SHLE } \\ \text { 340HNTN } & 18,830 & 19,145 & \text { DLMT } \\ \text { 361SLVN } & 19,145 & 19,240 & \text { DLMT } \\ \text { 361VIOL } & 19,240 & & \text { IMSN }\end{array}$


Table 3.--Geologic logs for wells in the study area--Continued

Rogers County, Oklahoma

Latitude: 361922

Owner:

Use of site: P

Altitude :

865
Eongitude: 954258

Local number: $21 \mathrm{~N}-15 \mathrm{E}-04$ CD 1 Completion date:

Depth of well: 1,370
Geologic-

unit code

325LBTT

325FRSC

325 CHRK

325CHRK

325CHRK

328MRRW

333MRMC

331BOON

337STJO

339NRTV

341CTNG

367CTTR
Depth to Depth to

$$
\text { top bottom }
$$

0
53

146

508

663

906

1,059

1,159

1,229

1,241

1,253

1,308

$$
\begin{array}{r}
53 \\
146 \\
508 \\
563 \\
906 \\
1,059 \\
1,159 \\
1,229 \\
1,241 \\
1,253 \\
1,308
\end{array}
$$

Lithology

SHLE

IMSN

SDSL

SNDS

SDSI

IMSH

IMSN

LMSN

LMSN

SHLE

SHLE

DLMT 
Table 3.--Geologic logs for wells in the study area--Continued

Seminole County, Oklahoma

Latitude: 351022

Owner: LIVINGSTON

Use of site: P

Altitude: $\quad 865$
Longitude: 963936

Local number: 08N-06E-15 ABB 1 Completion date:

Depth of well: 4,880

$\begin{array}{lrrc}\begin{array}{l}\text { Geologic- } \\ \text { unit code }\end{array} & \begin{array}{r}\text { Depth to } \\ \text { top }\end{array} & \begin{array}{r}\text { Depth to } \\ \text { bottom }\end{array} & \text { Lithology } \\ \text { 322VMOS } & 0 & 590 & \text { SDSL } \\ \text { 324BLCT } & 590 & 600 & \text { LMSN } \\ \text { 323NLBL } & 600 & 1,060 & \text { SDSL } \\ \text { 323CFVL } & 1,060 & 1,300 & \text { SDSL } \\ \text { 323SMNL } & 1,300 & 1,500 & \text { SHLE } \\ \text { 325HLDV } & 1,500 & 1,730 & \text { SHLE } \\ \text { 325WWOK } & 1,730 & 2,020 & \text { SDSL } \\ \text { 325WTMR } & 2,020 & 2,120 & \text { SHLE } \\ \text { 325CLVN } & 2,120 & 2,450 & \text { SDSL } \\ \text { 325CHRK } & 2,450 & 3,260 & \text { SHLE } \\ \text { 333MRMC } & 3,260 & 3,410 & \text { SHLE } \\ \text { 330MSSP } & 3,410 & 3,590 & \text { LMSN } \\ \text { 341WDFD } & 3,590 & 3,727 & \text { SHLE } \\ \text { 340MSNR } & 3,727 & 3,730 & \text { SNDS } \\ \text { 361SLVN } & 3,730 & 3,810 & \text { SHLE } \\ \text { 361VIOL } & 3,810 & 3,880 & \text { LMSN } \\ \text { 364SMPS } & 3,880 & 3,940 & \text { DLMT } \\ \text { 364SMPS } & 3,940 & 3,980 & \text { SNDS } \\ \text { 364SMPS } & 3,980 & 4,015 & \text { DLMT } \\ \text { 364SMPS } & 4,015 & 4,250 & \text { SDSL } \\ \text { 364SMPS } & 4,250 & 4,640 & \text { SDSL } \\ \text { 367ABCK } & 4,610 & & \text { DLMT }\end{array}$


Table 3.--Geologic logs for wells in the study area--Continued

Sequoyah County, Oklahoma

Latitude: 352757 Owner : SNOW Use of site: $P$ Altitude: 524
Long1tude: 945526

Local number: $12 N-22 E-36$ CD 1 Completion date: 12/03/1959

Depth of well: 1,718
Geologicunit code

\section{ATCK}

326ATCK

326ATCK

328WPCK

327UNVL

333MRMC

34IWDFD

340MSNR

34OHNTN

36ISLVN

36IVIOL

364SMPS

367ABCK
Depth to
top

130

300

430

560

730

795

950

960

990

1,245

1,280

1,340

1,620
300

430

560

730

795

950

960

990

1,245

1,280

1,340

1,620
Lithology

SDSL

SNDS

SHLE

IMSN

IMSH

IMSN

SHLE

SNDS

LMDM

SHLE

IMSN

IMDM

DLMT 
Table 3.--Geologic logs for wells in the study area--Continued

Sequoyah County, Oklahoma.

Latitude: 352958

Owner: CHEEK

Use of site: $P$

Altitude :

595
Longitude: 944135

Local number: $12 \mathrm{~N}-25 \mathrm{E}-19 \mathrm{CAA} 1$

Completion date: $12 / 11 / 1958$

Depth of well: 3,504

$\begin{array}{lrrc}\begin{array}{l}\text { Geologic- } \\ \text { unit code }\end{array} & \begin{array}{r}\text { Depth } \\ \text { top }\end{array} & \begin{array}{r}\text { Depth to } \\ \text { bottom }\end{array} & \text { Lithology } \\ \text { 326ATCK } & 170 & 340 & \text { SHLE } \\ \text { 326ATCK } & 340 & 820 & \text { SDSL } \\ \text { 326ATCK } & 820 & 1,060 & \text { SDSL } \\ \text { 326ATCK } & 1,060 & 1,180 & \text { SNDS } \\ \text { 326ATCK } & 1,180 & 1,600 & \text { SHLE } \\ \text { 326ATCK } & 1,600 & 1,740 & \text { SHLE } \\ \text { 326ATCK } & 1,740 & 1,800 & \text { SNDS } \\ \text { 326ATCK } & 1,800 & 2,140 & \text { SHLE } \\ \text { 326ATCK } & 2,140 & 2,400 & \text { SNDS } \\ \text { 326ATCK } & 2,400 & 2,500 & \text { SDSL } \\ \text { 328WPCK } & 2,500 & 2,690 & \text { LMSH } \\ \text { 328WPCK } & 2,690 & 2,710 & \text { LMSN } \\ \text { 327UNVI } & 2,710 & 2,750 & \text { LMSN } \\ \text { 331PTKN } & 2,750 & 2,810 & \text { LMSN } \\ \text { 331BOON } & 2,810 & 2,915 & \text { LMSN } \\ \text { 341WDFD } & 2,915 & 2,955 & \text { SHLE } \\ \text { 340HNTN } & 2,955 & 3,230 & \text { LMSN } \\ \text { 361SLVN } & 3,230 & 3,260 & \text { SHLE } \\ \text { 361VIOL } & 3,260 & 3,315 & \text { LMSN } \\ \text { 364SMPS } & 3,315 & & \text { SLSN }\end{array}$


Table 3.--Geologic logs for wells in the study area--Continued

Stephens County, Oklahoma

Latitude: 343533

Owner: FBRGUSON

Use of site: $P$

Altitude: 1,105
Long1tude: 980323

Local number: 01N-08W-05 AA 1 Completion date: $12 / 05 / 1971$

Depth of well: 9,152

\begin{tabular}{lrr|c}
$\begin{array}{c}\text { Geologic- } \\
\text { unit code }\end{array}$ & $\begin{array}{r}\text { Depth } \\
\text { top }\end{array}$ & $\begin{array}{r}\text { Depth to } \\
\text { bottom }\end{array}$ & Lithology \\
318HNSS & 0 & 520 & SHLE \\
318HNSS & 520 & 790 & SDSL \\
318HNSS & 790 & 2,730 & SHLE \\
319PNTC & 2,730 & 4,112 & SHLE \\
321CSCO & 4,112 & 4,809 & SHLE \\
321CSCO & 4,809 & 6,120 & SDSL \\
321CSCO & 6,120 & 6,370 & SNDS \\
323HXBR & 6,370 & 6,890 & SHLE \\
323HXBR & 6,890 & 7,225 & LMSN \\
323HXBR & 7,225 & 7,867 & CGLM \\
323HXBR & 7,867 & 8,270 & CGLM \\
367ABCK & 8,270 & 8,901 & LMSN \\
367ABCK & 8,901 & 9,112 & LMSN \\
367ABCK & 9,112 & & LMSN
\end{tabular}

Stephens County, Oklahoma

Latitude: 342743

Owner: KERR MCGEE

Use of site: P

Altitude: 1,133
Longitude: 980419

Local number: 01S-08W-19 AA 1

Completion date: 05/31/1966

Depth of well: 8,515

$\begin{array}{lccc}\begin{array}{c}\text { Geologic- } \\ \text { unit code }\end{array} & \begin{array}{c}\text { Depth to } \\ \text { top }\end{array} & \begin{array}{c}\text { Depth to } \\ \text { bottom }\end{array} & \text { Lithology } \\ \text { 323HXBR } & 3,000 & 3,050 & \text { SNDS } \\ \text { 323HXBR } & 3,050 & 3,100 & \text { SHLE } \\ \text { 323HXBR } & 3,100 & 3,210 & \text { SNDS } \\ \text { 323HXBR } & 3,210 & 3,250 & \text { IMSN } \\ \text { 323HXBR } & 3,250 & 3,400 & \text { CGLM } \\ \text { 367ABCK } & 3,400 & 6,185 & \text { DLMT } \\ \text { 367ABCK } & 6,185 & 7,185 & \text { IMSN } \\ \text { 367ABCK } & 7,185 & 7,350 & \text { IMSN } \\ \text { 367ABCK } & 7,350 & 7,395 & \text { LMSN } \\ \text { 367ABCK } & 7,395 & 7,965 & \text { IMSN } \\ \text { 367ABCK } & 7,965 & 8,165 & \text { SNDS } \\ \text { 367ABCK } & 8,165 & 8,225 & \text { SHLE } \\ \text { 371REGN } & 8,225 & 8,408 & \text { SNDS } \\ \text { 371REGN } & 8,408 & 8,500 & \text { SHLI } \\ \text { 370CMBR } & 8,500 & & \text { GRNT }\end{array}$


Table 3.--Geologic logs for wells in the study area--Continued

Texas County, Oklahoma

Latitude: 364100

Owner: PHILLIPS

Use of site: T

Altitude: $\quad 3,648$
Longitude: 1020015

Local number: 03N-10ECM-32 ACC

Completion date: $05 / 06 / 1955$

Depth of well: 6,473
Geologicunit code

1210GLL

23IDCKM

31 IDCRK

317CMRN

313BLIN

310GLRT

318 HNSS

318 HNSS

31OPRMN

32OPSLV

32OPSLV

32OPSLV

32OPSLV

32OPSLV

333SGVV

330MSSP

36IVIOL

364SMPS
Depth to Depth to top bottom

0
287
380

415

780

970

1,120

1,512

1,560

2,930

4,150

4,475

4,800

5,350

5,440

5,640

6,260

6,320
287

380

415

780

970

1,120

1,512

1,560

2,930

4,150

4,475

4,800

5,350

5,440

5,640

6,260

6,320
Lithology

SDGL

SHLE

GPSM

SHLE

GPSM

SNDS

SHLE

ANDR

SHLE

LMSH

SHLE

LMSN

SHLE

SNDS

LMSN

LMSN

DLMT

DLMT 
Table 3.--Geologic logs for wells in the study area--Continued

Tulsa County, Oklahoma

Lat1tude: 360519

Owner: KNOCH

Use of site: P

Altitude:
822
Long1tude: 960715

Local number: 19N-11E-34 AA 1

Completion date:

Depth of well: 2,897

\begin{tabular}{lrr|c}
$\begin{array}{l}\text { Geologic- } \\
\text { un1t code }\end{array}$ & $\begin{array}{r}\text { Depth } \\
\text { top }\end{array}$ & $\begin{array}{r}\text { Depth to } \\
\text { bottom }\end{array}$ & Lithology \\
323CFVL & 0 & 564 & SHLE \\
323CCKB & 564 & 572 & LMSN \\
325MRMN & 572 & 856 & SHLE \\
325MRMN & 856 & 905 & LMSN \\
325MRMN & 905 & 1,034 & SHLE \\
325MRMN & 1,034 & 1,078 & LMSN \\
325CHRK & 1,078 & 1,778 & SHLE \\
326ATCK & 1,778 & 1,878 & SHLE \\
330MSSP & 1,878 & 2,060 & LMSN \\
330MSSP & 2,060 & 2,090 & SHLE \\
341CTNG & 2,090 & 2,118 & SHLE \\
364SMPS & 2,118 & 2,282 & SDSL \\
367ABCK & 2,282 & 2,882 & DLMT \\
400PCMB & 2,882 & & GRNT
\end{tabular}


Table 3.--Geologic logs for wells in the study area--Continued

Wagoner County, Oklahoma

Latitude: 355604

Owner: LOVETT Use of site: P Altitude:
Longitude: 963519

Local number: $17 \mathrm{~N}-16 \mathrm{E}-22$ DBD 1 Completion date: 06/09/1934 Depth of well: 1,600

$\begin{array}{lrrc}\begin{array}{l}\text { Geologic- } \\ \text { unit code }\end{array} & \begin{array}{r}\text { Depth } \\ \text { top }\end{array} & \begin{array}{c}\text { Depth to } \\ \text { bottom }\end{array} & \text { L1thology } \\ \text { 325BGGY } & 0 & 165 & \text { SHLE } \\ \text { 325CHRK } & 165 & 205 & \text { SHLE } \\ \text { 325CHRK } & 205 & 612 & \text { SDSL } \\ \text { 325HRSR } & 612 & 775 & \text { LMSH } \\ \text { 324STRN } & 775 & 925 & \text { SDSL } \\ \text { 328WPCK } & 925 & 1,003 & \text { LMSH } \\ \text { 328WPCK } & 1,003 & 1,008 & \text { SNDS } \\ \text { 331PTKN } & 1,008 & 1,038 & \text { LMSH } \\ \text { 332FTVL } & 1,038 & 1,105 & \text { SHLE } \\ \text { 333DLCK } & 1,105 & 1,124 & \text { SHLE } \\ \text { 333MRMC } & 1,124 & 1,288 & \text { LMSH } \\ \text { 339KDRK } & 1,288 & 1,320 & \text { SHLE } \\ \text { 341CTNG } & 1,320 & 1,352 & \text { SHLE } \\ \text { 364SMPS } & 1,352 & 1,515 & \text { SDSL } \\ \text { 364BRGN } & 1,515 & 1,584 & \text { SDSL } \\ \text { 367ABCK } & 1,584 & & \text { DLMT }\end{array}$


Table 3.--Geologic logs for wells in the study area--Continued

Wagoner County, Oklahoma

Lat1tude: 355413

Owner: SHBLLY

Use of site: P

Alt1tude :
565
Long1tude: 953800

Local number: $17 \mathrm{~N}-16 \mathrm{E}-32$ CCA 1

Completion date: $12 / 22 / 1980$

Depth of well: 1,581

\begin{tabular}{lrc|c}
$\begin{array}{l}\text { Geolog1c- } \\
\text { unit code }\end{array}$ & $\begin{array}{r}\text { Depth } \\
\text { top }\end{array}$ & $\begin{array}{c}\text { Depth to } \\
\text { bottom }\end{array}$ & Lithology \\
325CHRK & 588 & 622 & SNDS \\
325CHRK & 622 & 760 & SHLE \\
324STRN & 760 & 784 & LMSN \\
324STRN & 784 & 804 & SHLB \\
324STRN & 804 & 828 & LMSH \\
324STRN & 828 & 860 & SHLE \\
324STRN & 860 & 886 & SNDS \\
324STRN & 886 & 906 & SHLE \\
327UNVL & 906 & 944 & LMSN \\
327UNVL & 944 & 950 & SNDS \\
331PTKN & 950 & 976 & LMSN \\
331PTKN & 976 & 997 & LMSH \\
332FTVL & 997 & 1,088 & SHLE \\
333DLCK & 1,088 & 1,146 & SHLE \\
333MRMC & 1,146 & 1,250 & LMSN \\
339KDRK & 1,250 & 1,308 & SHLE \\
341CTNG & 1,308 & 1,325 & SHLE \\
340MSNR & 1,325 & 1,336 & SNDS \\
361SLVN & 1,336 & 1,340 & SHLE \\
364SMPS & 1,340 & 1,351 & SNDS \\
364SMPS & 1,351 & 1,366 & SHLE \\
364TYNR & 1,366 & 1,402 & SNDS \\
364SMPS & 1,402 & 1,467 & SHLE \\
364TYNR & 1,467 & 1,496 & SNDS \\
364SMPS & 1,496 & 1,504 & SHLE \\
364BRGN & 1,504 & 1,524 & SNDS \\
364SMPS & 1,524 & 1,534 & SDSL \\
367ABCK & 1,534 & & DLMT \\
& & & \\
& & & \\
& & & \\
& & &
\end{tabular}


Table 3.--Geologic logs for wells in the study area--Continued

Washington County, Oklahoma

Latitude: 365825

Owner: THOMASON

Use of site: P

Altitude :
750
Longitude: 955250

Local number: 29N-13E-22 DDB 1 Completion date: $02 / 03 / 1917$

Depth of well: 3,175

\begin{tabular}{|c|c|c|c|}
\hline $\begin{array}{l}\text { Geologic- } \\
\text { unit code }\end{array}$ & $\begin{array}{c}\text { Depth to } \\
\text { top }\end{array}$ & $\begin{array}{l}\text { Depth to } \\
\text { bottom }\end{array}$ & Lithology \\
\hline 323MSSR & 0 & 50 & LMSH \\
\hline 323MSSR & 50 & 110 & SDSL \\
\hline 323MSSR & 110 & 200 & SHLE \\
\hline 323MSSR & 200 & 210 & SDSL \\
\hline 323MSSR & 210 & 290 & SHLE \\
\hline 323HGSR & 290 & 300 & IMSN \\
\hline 323MSSR & 300 & 340 & SNDS \\
\hline 323MSSR & 340 & 550 & SHLE \\
\hline 325MRMN & 550 & 565 & IMSN \\
\hline 325MRMN & 565 & 638 & SHLE \\
\hline 325MRMN & 638 & 665 & IMSH \\
\hline 325MRMN & 665 & 680 & IMSN \\
\hline 325MRMN & 680 & 760 & SHLE \\
\hline 325MRMN & 760 & 785 & LMSN \\
\hline 325MRMN & 785 & 795 & SHLE \\
\hline 325MRMN & 795 & 805 & SNDS \\
\hline 325MRMN & 805 & 905 & SHLE \\
\hline 325MRMN & 905 & 975 & LMSH \\
\hline 325MRMN & 975 & 1,005 & IMSH \\
\hline 325CHRK & 1,005 & 1,010 & COAL \\
\hline 325 CHRK & 1,010 & 1,110 & SHLE \\
\hline 325CHRK & 1,110 & 1,135 & SNDS \\
\hline 325CHRK & 1,135 & 1,235 & SHLE \\
\hline $325 \mathrm{CHRK}$ & 1,235 & 1,275 & SNDS \\
\hline $325 \mathrm{CHRK}$ & 1,275 & 1,325 & SHLE \\
\hline $325 \mathrm{CHRK}$ & 1,325 & 1,355 & LMSN \\
\hline $325 \mathrm{CHRK}$ & 1,355 & 1,395 & SHLE \\
\hline $325 \mathrm{CHRK}$ & 1,395 & 1,485 & SNDS \\
\hline 330MSSP & 1,485 & 1,685 & LMSN \\
\hline 338RSPG & 1,685 & 1,750 & LMSN \\
\hline 341WDFD & 1,750 & 1,815 & SHLE \\
\hline 367CRJF & 1,815 & 2,270 & DLMT \\
\hline 367RBDX & 2,270 & 2,390 & SNDS \\
\hline 367ABCK & 2,390 & 2,610 & DLMT \\
\hline 37IREGN & 2,610 & 2,720 & SHLE \\
\hline 400PCMB & 2,720 & & GRNT \\
\hline
\end{tabular}


Table 3.--Geologic logs for wells in the study area--Continued

Washita County, OkIahoma

Latitude: 350723

Owner: NO. AMER.

Use of site: $P$

Altitude: $\quad 1,602$
Iongitude: 990353

Local number: 08N-18W-35 DAB 1

Completion date:

Depth of well: 15,013

\begin{tabular}{|c|c|c|c|}
\hline $\begin{array}{l}\text { Geologic- } \\
\text { unit code }\end{array}$ & $\begin{array}{l}\text { Depth to } \\
\text { top }\end{array}$ & $\begin{array}{l}\text { Depth to } \\
\text { bottom }\end{array}$ & Lithology \\
\hline 310PRMN & 200 & 1,270 & SHLE \\
\hline 319PNTC & 1,270 & 1,675 & SHLE \\
\hline 319PNTC & 1,675 & 1,840 & SHLE \\
\hline 367ABCK & 1,840 & 2,170 & DLMT \\
\hline 367ABCK & 2,170 & 2,440 & DLMT \\
\hline $367 \mathrm{ABCK}$ & 2,440 & 2,590 & IMSN \\
\hline $367 \mathrm{ABCK}$ & 2,590 & 2,780 & IMDM \\
\hline 364SMPS & 2,780 & 2,970 & IMSH \\
\hline 364SMPS & 2,970 & 3,220 & SHLE \\
\hline 364SMPS & 3,220 & 3,270 & SLSN \\
\hline 364SMPS & 3,270 & 3,700 & SHLE \\
\hline 361 VIOL & 3,700 & 4,200 & LMSN \\
\hline 361 VIOL & 4,200 & 4,250 & LMSN \\
\hline 361 VIOL & 4,250 & 4,500 & LMSN \\
\hline 36IVIOL & 4,500 & 4,610 & LMSN \\
\hline 361 VIOL & 4,610 & 4,780 & LMSN \\
\hline 361VIOL & 4,780 & 5,010 & IMSSN \\
\hline 361 IOI & 5,010 & 5,280 & IMSN \\
\hline 364 SMPS & 5,280 & 5,590 & SHLE \\
\hline 364SMPS & 5,590 & 5,900 & SHLE \\
\hline 364SMPS & 5,900 & 6,000 & IMSN \\
\hline 364SMPS & 6,000 & 6,480 & LMSH \\
\hline 367ABCK & 6,480 & 6,590 & IMSN \\
\hline $367 \mathrm{ABCK}$ & 6,590 & 6,700 & LMSN \\
\hline $367 \mathrm{ABCK}$ & 6,700 & 7,220 & IMDM \\
\hline 332CSTR & 7,220 & 7,320 & IMSN \\
\hline 332CSTR & 7,320 & 7,480 & SHLE \\
\hline 332CSTR & 7,480 & 7,590 & IMSN \\
\hline 332CSTR & 7,590 & 7,820 & SHLE \\
\hline 332CSTR & 7,820 & 8,820 & LMSH \\
\hline 332CSTR & 8,820 & 8,950 & IMSN \\
\hline 332CSTR & 8,950 & 10,280 & SHLE \\
\hline 338 SCMR & 10,280 & 10,890 & LMSN \\
\hline 338SCMR & 10,890 & 11,420 & IMSN \\
\hline 34IWDFD & 11,420 & 12,030 & SHLE \\
\hline 340HNTN & 12,030 & 12,590 & LMSN \\
\hline 34OHNTN & 12,590 & 13,890 & LMSN \\
\hline 357CMNL & 13,890 & 14,180 & IMSN \\
\hline
\end{tabular}


Table 3.--Geologic logs for wells in the study area--Continued

Washita County, Oklahoma--Continued

Latitude: 350723

Owner: NO. AMER.

Use of site: $P$

Altitude: $\quad 1,602$
Longitude: 990353

Local number: 08N-18W-35 DAB 1 Completion date:

Depth of well: 15,013

$\begin{array}{lccc}\begin{array}{c}\text { Geologic- } \\ \text { unit code }\end{array} & \begin{array}{c}\text { Depth to } \\ \text { top }\end{array} & \begin{array}{c}\text { Depth to } \\ \text { bottom }\end{array} & \text { Lithology } \\ \text { 36ISLVN } & 14,180 & 14,300 & \text { SHLE } \\ \text { 36ISLVN } & 14,300 & 14,410 & \text { SHLE } \\ \text { 361VIOL } & 14,410 & 14,730 & \text { IMSN } \\ \text { 364SMPS } & 14,730 & 14,840 & \text { SHLE } \\ \text { 364SMPS } & 14,840 & 14,990 & \text { IMSH } \\ \text { 364SMPS } & 14,990 & 16,010 & \text { SNDS } \\ \text { 364SMPS } & 15,010 & & \text { QRTZ }\end{array}$


Table 3.--Geologic logs for wells in the study area--Continued

Woods County, Oklahoma

Latitude: 364257

Owner: ATLANTIC

Use of site: T

Altitude: $\quad 1,465$
Longitude: 984616

Local number: $26 \mathrm{~N}-15 \mathrm{~W}-24 \mathrm{CB}$

Completion date: $07 / 31 / 1948$

Depth of well: 7,370

$\begin{array}{lr}\begin{array}{l}\text { Geologio- } \\ \text { unit code }\end{array} & \begin{array}{r}\text { Depth } \\ \text { top }\end{array} \\ \text { 310PRMN } & 0 \\ \text { 319WFMP } & 2,350 \\ \text { 322WBNS } & 3,290 \\ \text { 322WBNS } & 3,684 \\ \text { 322SHWN } & 3,818 \\ \text { 322SHWN } & 4,460 \\ \text { 322DGLS } & 4,580 \\ \text { 322DGLS } & 4,820 \\ \text { 323HXBR } & 5,000 \\ \text { 330MSSP } & 5,700 \\ \text { 341WDFD } & 6,470 \\ \text { 340HNTN } & 6,525 \\ \text { 361SLVN } & 6,552 \\ \text { 361VIOL } & 6,675 \\ \text { 364SMPS } & 6,824 \\ \text { 364SMPS } & 6,970 \\ \text { 367ABCK } & 7,090\end{array}$

Depth to bottom

Lithology

2,350

SHLE

3,290

3,684

3,818

4,460

4,580

4,820

5,000

5,700

6,470

6,525

6,552

6,675

6,824

6,970

IMSH

SHLE

IMSN

SHLE

IMSN

SHLE

SNDS

IMSH

IMSN

SHLB

IMSN

SHLE

IMSN

7,090

SNDS

SHLE

DLMT 
Table 3.--Geologic logs for wells in the study area--Continued

\section{Woodward County, Oklahoma}

Latitude: 362941

Owner: SINCLAIR

Use of site: $T$

Altitude: $\quad 2,010$
Longitude: 991557

Local number: 23N-19W-06 DD

Completion date: 05/07/1951

Depth of well: 10,327

$\begin{array}{lrrr}\begin{array}{l}\text { Geologic- } \\ \text { unit code }\end{array} & \begin{array}{r}\text { Depth } \\ \text { top }\end{array} & \begin{array}{r}\text { Depth to } \\ \text { bottom }\end{array} & \text { Lithology } \\ \text { 112TRRCH } & 120 & 150 & \text { SAND } \\ \text { 310WTRS } & 150 & 280 & \text { SNDS } \\ \text { 310PRMN } & 280 & 2,022 & \text { SHLE } \\ \text { 310WLNG } & 2,022 & 3,020 & \text { ANDR } \\ \text { 310WLNG } & 3,020 & 3,236 & \text { SHLE } \\ \text { 319CHSE } & 3,236 & 3,710 & \text { LMSN } \\ \text { 319CCGV } & 3,710 & 4,185 & \text { LMSN } \\ \text { 322VRGL } & 4,185 & 4,820 & \text { LMSN } \\ \text { 322SHWN } & 4,820 & 5,228 & \text { SHLE } \\ \text { 322ORED } & 5,228 & 5,240 & \text { LMSN } \\ \text { 322SHWN } & 5,240 & 5,550 & \text { SHLE } \\ \text { 322SHWN } & 5,550 & 5,610 & \text { SNDS } \\ \text { 321TRNT } & 5,610 & 5,695 & \text { LMSN } \\ \text { 322DGLS } & 5,695 & 5,910 & \text { SHLE } \\ \text { 323HXBR } & 5,910 & 6,025 & \text { SNDS } \\ \text { 323MSSR } & 6,025 & 6,364 & \text { SHLE } \\ \text { 323KSSC } & 6,364 & 6,415 & \text { SNDS } \\ \text { 323HXBR } & 6,415 & 6,480 & \text { SHLE } \\ \text { 323HGSR } & 6,480 & 6,492 & \text { LMSN } \\ \text { 323CCKB } & 6,492 & 6,636 & \text { LMSN } \\ \text { 325FRSC } & 6,636 & 6,698 & \text { LMSN } \\ \text { 325DSMS } & 6,698 & 8,180 & \text { LMSH } \\ \text { 330MSSP } & 8,180 & 9,303 & \text { LMSN } \\ \text { 341WDFD } & 9,303 & 9,320 & \text { SHLE } \\ \text { 340HNTN } & 9,320 & 9,525 & \text { LMSN } \\ \text { 361SLVN } & 9,525 & 9,560 & \text { SHLE } \\ \text { 361VIOL } & 9,560 & 9,703 & \text { DLMT } \\ \text { 364SMPS } & 9,703 & 9,870 & \text { DLMT } \\ \text { 364SMPS } & 9,870 & 9,950 & \text { SNDS } \\ \text { 364SMPS } & 9,950 & 10,047 & \text { SHLE } \\ \text { 364SMPS } & 10,047 & 10,070 & \text { DLMT } \\ \text { 364SMPS } & 10,070 & 10,125 & \text { SNDS } \\ \text { 364SMPS } & 10,125 & 10,295 & \text { DLMT } \\ \text { 367ABCE } & 10,295 & & \text { DLMT } \\ & & & \end{array}$


Table 3.--Geologic logs for wells in the study

area--Continued

Carson County, Texas

Latitude: 352030

Owner: WHITTENBURG

Use of site: $T$

Altitude: $\quad 3,500$
Long1tude: 1013000

Local number: GIBSON BLK M4 SEC 4 Completion date: 08/01/1953

Depth of well: 9,311

\begin{tabular}{lrr|c}
$\begin{array}{l}\text { Geologic- } \\
\text { unit code }\end{array}$ & $\begin{array}{r}\text { Depth } \\
\text { top }\end{array}$ & $\begin{array}{c}\text { Depth to } \\
\text { bottom }\end{array}$ & Lithology \\
1210GLI & 0 & 805 & SDGL \\
31OPRMN & 805 & 1,520 & SDSL \\
313BLIN & 1,520 & 1,740 & ANDR \\
317CMRN & 1,740 & 1,820 & DLMT \\
31OGLRT & 1,820 & 1,850 & SNDS \\
318HNSS & 1,850 & 2,985 & EVPR \\
318HNSS & 2,985 & 3,245 & ANDR \\
318LNRD & 3,245 & 3,505 & SHLR \\
318LNRD & 3,505 & 3,570 & ANDR \\
318WCHT & 3,570 & 4,145 & DLMT \\
319WFMP & 4,145 & 4,694 & DLMT \\
319WFMP & 4,694 & 5,900 & LMSN \\
320PSLV & 5,900 & & ARRS
\end{tabular}

Dallam County, Texas

Latitude: 360630

Owner: SKELLY

Use of site: $T$

Altitude: 4,327
Long1tude: 1025300

Locel number: CSS BLK 5 SEC 58

Completion date: 11/07/1958

Depth of well: 8,085

\begin{tabular}{lrr|c}
$\begin{array}{l}\text { Geologic- } \\
\text { unit code }\end{array}$ & $\begin{array}{r}\text { Depth to } \\
\text { top }\end{array}$ & $\begin{array}{r}\text { Depth to } \\
\text { bottom }\end{array}$ & Lithology \\
120TRTR & 0 & 438 & LMSD \\
220JRSC & 438 & 640 & SDSL \\
230TRSC & 640 & 1,494 & SDSL \\
310PRMN & 1,494 & 1,900 & SDSL \\
313BLIN & 1,900 & 2,114 & ANDR \\
310GLRT & 2,114 & 2,186 & SNDS \\
318HNSS & 2,186 & 2,700 & SDSL \\
318LNRD & 2,700 & 2,858 & EVPR \\
318LNRD & 2,858 & 3,296 & SDSL \\
319WFMP & 3,296 & 4,792 & ARKS \\
319WFMP & 4,792 & 5,360 & LMSH \\
320PSLV & 5,360 & 8,010 & ARKS \\
333MRMC & 8,010 & & LMSN
\end{tabular}


Table 3.--Geologic logs for wells in the study area--Continued

Dallam County, Texas

Lat1tude: 361940

Owner: CONTINENTAL Use of site: $P$ Altitude: 4,415
Long1tude: 1025245

Local number: CSS BLK.81,7

Completion date:

Depth of well: 7,808

$\begin{array}{lccc}\begin{array}{l}\text { Geologic- } \\ \text { unit code }\end{array} & \begin{array}{r}\text { Depth to } \\ \text { top }\end{array} & \begin{array}{c}\text { Depth to } \\ \text { bottom }\end{array} & \text { Lithology } \\ \text { 313BLIN } & 1,440 & 1,970 & \text { ANDR } \\ \text { 318CLFK } & 1,970 & 2,565 & \text { SHLE } \\ \text { 310PRMN } & 2,565 & 4,900 & \text { ARKS } \\ \text { 310PMPV } & 4,900 & 6,780 & \text { ARKS } \\ \text { 328MRRW } & 6,780 & 7,380 & \text { ARKS } \\ \text { 367ELBG } & 7,380 & 7,770 & \text { DLMT } \\ \text { 400GRNT } & 7,770 & & \text { GRNT }\end{array}$

Dallam County, Texas

Lat1tude: 362645

Owner: HUMBLE OIL C Use of site: $P$ Altitude: 3,854
Longitude: 1021540

Local number: H TC RR BLK. 50, 16 Completion date:

Depth of well: 6,214

$\begin{array}{lrcc}\begin{array}{l}\text { Geologio- } \\ \text { unit code }\end{array} & \begin{array}{r}\text { Depth } \\ \text { top }\end{array} & \begin{array}{c}\text { Depth to } \\ \text { bottom }\end{array} & \text { L1thology } \\ \text { 313GDLP } & 610 & 1,120 & \text { ANDR } \\ \text { 318CLFR } & 1,120 & 1,755 & \text { SDSL } \\ \text { 318WCHT } & 1,755 & 2,165 & \text { SDSL } \\ \text { 318WCHT } & 2,165 & 2,970 & \text { ANDR } \\ \text { 319WFMP } & 2,970 & 3,240 & \text { DLMT } \\ \text { 321CSCO } & 3,240 & 3,470 & \text { LMSN } \\ \text { 321CNYN } & 3,470 & 3,810 & \text { LMSN } \\ \text { 324STRN } & 3,810 & 4,200 & \text { LMSN } \\ \text { 326ATCR } & 4,200 & 4,380 & \text { LMSH } \\ \text { 328MRRW } & 4,380 & 4,750 & \text { LMSH } \\ \text { 332CSTR } & 4,750 & 4,835 & \text { LMSN } \\ \text { 333MRMC } & 4,835 & 5,450 & \text { LMSN } \\ \text { 361VIOL } & 5,450 & 5,515 & \text { LMSN } \\ \text { 364SMPS } & 5,515 & 5,600 & \text { SDSL } \\ \text { 367ABCR } & 5,600 & 6,200 & \text { IMDM } \\ \text { 400GRNT } & 6,200 & & \text { GRNT }\end{array}$


Table 3.--Geologic logs for wells in the study

area--Continued

Gray County, Texas

Latitude: 363030

Owner: PHILLIPS

Use of site: T

Altitude: 2,854
Long1tude: 1003730

Local number: H GN;BLK A-6 SBC 53

Completion date: $08 / 14 / 1972$

Depth of well: 13,594

\begin{tabular}{lrr|c}
$\begin{array}{l}\text { Geolog10- } \\
\text { un1t code }\end{array}$ & $\begin{array}{r}\text { Depth } \\
\text { top }\end{array}$ & $\begin{array}{r}\text { Depth to } \\
\text { bottom }\end{array}$ & L1thology \\
1210GLL & 0 & 340 & SDGL \\
310PRMN & 340 & 1,020 & SDSL \\
313BLIN & 1,020 & 1,180 & ANDR \\
318HNSS & 1,180 & 2,783 & BVPR \\
318HNSS & 2,783 & 3,150 & SHLE \\
310WLNG & 3,150 & 3,846 & ANDR \\
319WFMP & 3,845 & 4,223 & LMDM \\
310PMPV & 4,223 & 6,400 & ARKS \\
320PSLV & 6,400 & 11,200 & ARKS \\
320PSLV & 11,200 & 11,330 & CHRT \\
339RDRK & 11,330 & 11,350 & SNDS \\
340HNTN & 11,350 & 12,100 & LMSN \\
361SLVN & 12,100 & 12,250 & DMSH \\
361VIOL & 12,250 & 12,620 & LMSN \\
364SMPS & 12,620 & 13,040 & SDSL \\
367ABCK & 13,040 & & DLMT
\end{tabular}


Table 3.--Geologic logs for wells in the study area--Continued

Hansford County, Texas

Latitude: 361760

Owner: GULF OIL

Use of site: $T$

Altitude: $\quad 3,193$
Longitude: 1012400

Local number: H GN; BLK P, SEC 17 Completion date: $07 / 17 / 1968$

Depth of well: $\quad 9,440$

$\begin{array}{lrrc}\begin{array}{c}\text { Geolog10- } \\ \text { unit oode }\end{array} & \begin{array}{c}\text { Depth to } \\ \text { top }\end{array} & \begin{array}{c}\text { Depth to } \\ \text { bottom }\end{array} & \text { Lithology } \\ \text { 1210GLI } & 0 & 490 & \text { SDGL } \\ \text { 310PRMN } & 490 & 970 & \text { SNDS } \\ \text { 313BLIN } & 970 & 1,110 & \text { ANDR } \\ \text { 310GLRT } & 1,110 & 1,160 & \text { SNDS } \\ \text { 318HNSS } & 1,160 & 1,800 & \text { EVPR } \\ \text { 318HNSS } & 1,800 & 2,455 & \text { SHLE } \\ \text { 310WLNG } & 2,455 & 3,050 & \text { ANDR } \\ \text { 319WFMP } & 3,050 & 3,404 & \text { DIMT } \\ \text { 319WFMP } & 3,404 & 4,150 & \text { LMSN } \\ \text { 310PMPV } & 4,150 & 5,090 & \text { SHLE } \\ \text { 323MSSR } & 5,090 & 5,150 & \text { SNDS } \\ \text { 323MSSR } & 5,150 & 5,800 & \text { SHLE } \\ \text { 325DSMS } & 5,800 & 6,055 & \text { LMSN } \\ \text { 325DSMS } & 6,055 & 6,205 & \text { SHLE } \\ \text { 320PSLV } & 6,205 & 6,730 & \text { LMSH } \\ \text { 332CSTR } & 6,730 & 7,185 & \text { LMSH } \\ \text { 330MSSP } & 7,185 & 7,800 & \text { LMSN } \\ \text { 3380SGE } & 7,800 & 8,000 & \text { LMSN } \\ \text { 339KDRR } & 8,000 & 8,250 & \text { SDSI } \\ \text { 340HNTN } & 8,250 & 8,500 & \text { LMSN } \\ \text { 340HNTN } & 8,500 & 8,830 & \text { SDSI } \\ \text { 361VIOL } & 8,830 & 9,080 & \text { LMSN } \\ \text { 364SMPS } & 9,080 & 9,205 & \text { SDSL } \\ \text { 367ELBG } & 9,205 & & \text { DLMT }\end{array}$


Table 3.--Geologic logs for wells in the study area--Continued

\section{Hartley County, Texas}

Latitude: 364400

Owner: SHAMROCK OIL Use of site: P

Altitude: $\quad 4,031$
Longitude: 1024900

Local number: G M, LE, 7 Completion date:

Depth of well: 6,114
Geolog10- Depth to Depth to unit oode top bottom

313BLIN

318CLFK

318WCHT

318WCHT

319WFMP

32OPSLV

40OGRNT
1,306

1,865

2,624

3,400

3,890

4,770

6,065
1,865

2,624

3,400

3,890

4,770

6,055
Lithology

ANDR

SHLE

SDSL

SHLB

DIMT

ARKS

GRNT 
Table 3.--Geologic logs for wells in the study area--Continued

\section{Hartley County, Texas}

Latitude: 355330

Owner: PHILLIPS

Use of site: T

Altitude: $\quad 3,955$
Longitude: 1023500

Local number: T NO;BLK ITO SEC 18

Completion date: 12/02/1956

Depth of well: 9,166

$\begin{array}{lrcc}\begin{array}{c}\text { Geologic- } \\ \text { unit code }\end{array} & \begin{array}{r}\text { Depth } \\ \text { top }\end{array} & \begin{array}{c}\text { Depth to } \\ \text { bottom }\end{array} & \text { Lithology } \\ \text { 121OGLL } & 0 & 434 & \text { SDGL } \\ \text { 230TRSC } & 434 & 1,150 & \text { SHLE } \\ \text { 231DCKM } & 1,150 & 1,225 & \text { SNDS } \\ \text { 313GDLP } & 1,225 & 1,636 & \text { SDSL } \\ \text { 313BLIN } & 1,636 & 1,958 & \text { ANDR } \\ \text { 31OGLRT } & 1,958 & 2,290 & \text { SNDS } \\ \text { 318HNSS } & 2,290 & 2,826 & \text { EVPR } \\ \text { 31OPRMN } & 2,826 & 3,890 & \text { SDSL } \\ \text { 319WFMP } & 3,890 & 4,424 & \text { DLMT } \\ \text { 319WFMP } & 4,424 & 5,160 & \text { LMSN } \\ \text { 319WFMP } & 5,160 & 5,690 & \text { SDSL } \\ \text { 32OPSLV } & 5,690 & 6,265 & \text { SHLE } \\ \text { 323HXBR } & 6,265 & 6,618 & \text { ARKS } \\ \text { 320PSLV } & 6,618 & 7,420 & \text { LMSN } \\ \text { 320PSLV } & 7,420 & 8,432 & \text { SHLE } \\ \text { 333MRMC } & 8,432 & 8,510 & \text { LMSN } \\ \text { 333STLS } & 8,510 & 8,764 & \text { LMSN } \\ \text { 338OSGE } & 8,764 & 8,873 & \text { DLMT } \\ \text { 367ELBG } & 8,873 & 9,146 & \text { DLMT } \\ \text { 40OPCMB } & 9,146 & & \text { IGNS }\end{array}$


Table 3.--Geologic logs for wells in the study area--Continued

\section{Hemphill County, Texas}

Latitude: 354130

Owner: PHILLIPS

Use of site: $T$

Altitude: $\quad 2,488$
Iongitude: 1000530

Local number: G MMB A; BLK C,S 266

Completion date: 08/26/1967

Depth of well: 20,113

$\begin{array}{lr}\text { Geologio- } & \begin{array}{r}\text { Depth } \\ \text { top }\end{array} \\ \text { 121OGLL } & 0 \\ \text { 31OPRMN } & 257 \\ \text { 31OPRMN } & 600 \\ \text { 318HNSS } & 770 \\ \text { 317CMRN } & 2,028 \\ \text { 317CMRN } & 2,556 \\ \text { 31OWLNG } & 3,073 \\ \text { 319WFMP } & 4,160 \\ \text { 319WFMP } & 4,293 \\ \text { 319WFMP } & 5,910 \\ \text { 319WFMP } & 6,150 \\ \text { 31OPRMN } & 6,320 \\ \text { 31OPRMN } & 8,090 \\ \text { 322VRGL } & 8,490 \\ \text { 323MSSR } & 8,900 \\ \text { 323MSSR } & 9,330 \\ \text { 325DSMS } & 10,364 \\ \text { 325DSMS } & 11,320 \\ \text { 32OPSLV } & 12,950 \\ \text { 328MRRW } & 13,425 \\ \text { 328MRRW } & 13,525 \\ \text { 328MRRW } & 13,565 \\ \text { 328MRRW } & 15,040 \\ \text { 328MRRW } & 15,650 \\ \text { 332CSTR } & 15,890 \\ \text { 332CSTR } & 16,240 \\ \text { 332CSTR } & 16,720 \\ \text { 330MSSP } & 17,554 \\ \text { 338OSGE } & 18,440 \\ \text { 341WDFD } & 19,180 \\ \text { 34OHNTN } & 19,280 \\ \text { 361SLVN } & 19,800 \\ \text { 361VIOL } & 19,908 \\ & \end{array}$

Lithology

SDGL

SDSL

ANDR

BVPR

BVPR

SHLE

ANDR

DLMT

LMSN

SHLE

LMSN

SHLE

SDSL

SHLE

SDSL

SHLE

SDSL

ARKS

LMSH

SHLE

SNDS

SHLE

SDSL

SNDS

LMSN

SHLE

LMSN

IMSN

IMSN

SHLE

IMSN

SHLE

IMSN 
Table 3.--Geologic logs for wells in the study area--Continued

\section{Hutohinson County, Texas}

Latitude: 360100

Owner: SUN COMPANY

Use of site: T

Altitude: 3,154
Longitude: 1010900

Looal number: GB CNG; BLK R;SBC 77

Completion date: $01 / 17 / 1958$

Depth of well: 8,287

$\begin{array}{lrrc}\begin{array}{l}\text { Geologio- } \\ \text { unit code }\end{array} & \begin{array}{r}\text { Depth } \\ \text { top }\end{array} & \begin{array}{c}\text { Depth to } \\ \text { bottom }\end{array} & \text { Lithology } \\ \text { 1210GLL } & 0 & 514 & \text { SDGL } \\ \text { 31OPRMN } & 514 & 735 & \text { SNDS } \\ \text { 313BLIN } & 735 & 902 & \text { ANDR } \\ \text { 31OGLRT } & 902 & 955 & \text { SNDS } \\ \text { 317CMRN } & 955 & 2,180 & \text { EVPR } \\ \text { 317CMRN } & 2,180 & 2,526 & \text { SHLE } \\ \text { 310WLNG } & 2,526 & 3,490 & \text { ANDR } \\ \text { 319WFMP } & 3,490 & 3,820 & \text { DLMT } \\ \text { 319WFMP } & 3,820 & 4,690 & \text { LMSN } \\ \text { 320PSLV } & 4,690 & 5,400 & \text { SHLE } \\ \text { 320PSLV } & 5,400 & 6,230 & \text { SDSL } \\ \text { 323MSSR } & 6,230 & 6,490 & \text { LMSH } \\ \text { 325DSMS } & 6,490 & 7,633 & \text { SHLE } \\ \text { 328MRRW } & 7,633 & 8,125 & \text { SHLE } \\ \text { 328MRR } & 8,125 & 8,258 & \text { SNDS } \\ \text { 332CSTR } & 8,258 & & \text { LMSN }\end{array}$

\section{Hutohinson County, Texas}

Latitude: 353930

Owner: TEXAS OIL GA Use of site: $P$

Altitude: 3,111
Long1tude: 1013545

Local number: H TC RR, 46,89 Completion date:

Depth of well: 5,457

$\begin{array}{lccc}\begin{array}{l}\text { Geologic- } \\ \text { unit oode }\end{array} & \begin{array}{r}\text { Depth to } \\ \text { top }\end{array} & \begin{array}{c}\text { Depth to } \\ \text { bottom }\end{array} & \text { Lithology } \\ \text { 318WCHT } & 1,600 & 1,750 & \text { SDSL } \\ \text { 318WCHT } & 1,750 & 2,140 & \text { SHLE } \\ \text { 319WFMP } & 2,140 & 2,890 & \text { DMSH } \\ \text { 322VRGL } & 2,890 & 3,495 & \text { SHLE } \\ \text { 320PSLV } & 3,495 & 5,380 & \text { ARKS } \\ \text { 400GRNT } & 5,380 & 5,457 & \text { GRNT }\end{array}$


Table 3.--Geologic logs for wells in the study area-Continued

\section{Iipsoomb County, Texas}

Latitude: 361130

Owner: GULF OIL Use of site: $T$ Alt1tude: $\quad 2,785$
Iong1tude: 1003000

Local number: H TCRR; BLK 43;S 413 Completion date: $12 / 08 / 1949$

Depth of well: 14,278

\begin{tabular}{lrr|l}
$\begin{array}{l}\text { Geolog10- } \\
\text { unit code }\end{array}$ & $\begin{array}{r}\text { Depth } \\
\text { top }\end{array}$ & $\begin{array}{c}\text { Depth to } \\
\text { bottom }\end{array}$ & L1thology \\
1210GLI & 0 & 190 & SDGL \\
317CMRN & 190 & 620 & SNDS \\
317CMRN & 620 & 1,170 & SHLE \\
318LNRD & 1,170 & 1,430 & EVPR \\
318LNRD & 1,430 & 1,525 & SHLE \\
318LNRD & 1,525 & 1,850 & EVPR \\
318LNRD & 1,850 & 2,890 & SHLE \\
310WLNG & 2,890 & 3,575 & ANDR \\
319WFMP & 3,575 & 5,195 & LMSN \\
322VRGL & 5,195 & 6,160 & SHLE \\
320PSLV & 6,150 & 6,420 & SNDS \\
320PSLV & 6,420 & 7,515 & SDSL \\
325DSMS & 7,516 & 7,630 & LMSH \\
325DSMS & 7,630 & 8,190 & SDSL \\
325DSMS & 8,190 & 8,280 & LMSN \\
320PSLV & 8,280 & 9,225 & SHLE \\
320PSLV & 9,225 & 10,430 & SHLE \\
332CSTR & 10,430 & 10,900 & LMSH \\
332CSTR & 10,900 & 11,000 & SHLE \\
330MSSP & 11,000 & 12,670 & LMSN \\
3380SGE & 12,670 & 13,010 & LMSN \\
341WDFD & 13,010 & 13,060 & SHLE \\
340HNTN & 13,060 & 13,120 & LMSN \\
361SLVN & 13,120 & 13,180 & SHLE \\
361VIOL & 13,180 & 13,565 & LMSN \\
364SMPS & 13,565 & 13,860 & SDSL \\
367ELBG & 13,860 & & DLMT \\
& & &
\end{tabular}


Table 3.--Geologic logs for wells in the study area--Continued

Moore County, Texas

Lat1tude: 355630

Owner: SAC-MOBILE Use of site: T

Alt1tude: $\quad 3,416$
Long1tude: 1014300

Looal number: T NO,BLK 3T, SEC 183

Completion date:

Depth of well: 6,987

\begin{tabular}{|c|c|c|c|}
\hline $\begin{array}{l}\text { Geologio- } \\
\text { unit code }\end{array}$ & $\begin{array}{c}\text { Depth to } \\
\text { top }\end{array}$ & $\begin{array}{l}\text { Depth to } \\
\text { bottom }\end{array}$ & Isthology \\
\hline $\begin{array}{l}318 \mathrm{HNSS} \\
318 \mathrm{HNSS} \\
318 \mathrm{HNSS} \\
310 \mathrm{WLNG} \\
319 \mathrm{CHSE} \\
310 \mathrm{PMPV} \\
325 \mathrm{DSMS} \\
320 \mathrm{PSLV} \\
333 \mathrm{MRMC} \\
3380 \mathrm{SGE} \\
339 \mathrm{KDRK} \\
364 \mathrm{SMPS} \\
367 \mathrm{BLBG} \\
371 \mathrm{REGN} \\
400 \mathrm{PCMB}\end{array}$ & $\begin{array}{r}950 \\
1,385 \\
1,750 \\
2,185 \\
2,580 \\
2,890 \\
4,485 \\
4,920 \\
6,345 \\
5,685 \\
6,900 \\
6,060 \\
6,165 \\
6,943 \\
6,968\end{array}$ & $\begin{array}{l}1,385 \\
1,750 \\
2,185 \\
2,580 \\
2,890 \\
4,485 \\
4,920 \\
5,345 \\
5,685 \\
5,900 \\
6,060 \\
6,165 \\
6,943 \\
6,968\end{array}$ & $\begin{array}{l}\text { SHLEB } \\
\text { EVPR } \\
\text { SHLB } \\
\text { ANDR } \\
\text { DLMT } \\
\text { LMSN } \\
\text { ARKS } \\
\text { SHLE } \\
\text { LMSN } \\
\text { LMDM } \\
\text { LMSD } \\
\text { SHLE } \\
\text { DLMT } \\
\text { SNDS } \\
\text { IGNS }\end{array}$ \\
\hline
\end{tabular}


Table 3.--Geologic logs for wells in the study area--Continued

\section{Oohiltree County, Texas}

Latitude: 360530

Owner: GULF OIL

Use of site: T

Altitude: 3,077
Longitude: 1005730

Local number: J.J.BALINTINB SURVEY

Completion date: 01/25/1950

Depth of well: 11,640

\begin{tabular}{|c|c|c|c|}
\hline $\begin{array}{l}\text { Geologio- } \\
\text { unit code }\end{array}$ & $\begin{array}{c}\text { Depth to } \\
\text { top }\end{array}$ & $\begin{array}{l}\text { Depth to } \\
\text { bottom }\end{array}$ & Lithology \\
\hline 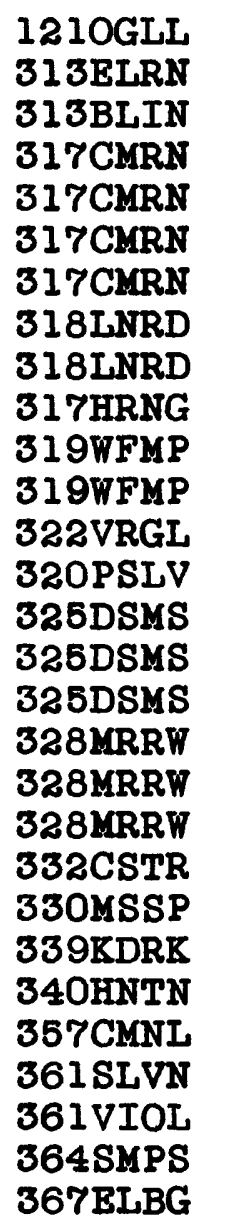 & $\begin{array}{r}0 \\
898 \\
940 \\
1,095 \\
1,160 \\
1,310 \\
1,500 \\
2,125 \\
2,490 \\
3,450 \\
3,825 \\
4,600 \\
5,580 \\
5,730 \\
6,880 \\
6,910 \\
7,325 \\
8,150 \\
8,260 \\
8,290 \\
8,950 \\
9,326 \\
10,505 \\
10,746 \\
10,820 \\
10,848 \\
10,922 \\
11,250 \\
11,402\end{array}$ & $\begin{array}{r}898 \\
940 \\
1,095 \\
1,160 \\
1,310 \\
1,500 \\
2,125 \\
2,490 \\
3,460 \\
3,825 \\
4,600 \\
5,580 \\
5,730 \\
6,880 \\
6,910 \\
7,325 \\
8,150 \\
8,260 \\
8,290 \\
8,950 \\
9,326 \\
10,505 \\
10,745 \\
10,820 \\
10,848 \\
10,922 \\
11,250 \\
11,402\end{array}$ & $\begin{array}{l}\text { SDGL } \\
\text { SNDS } \\
\text { ANDR } \\
\text { SHLE } \\
\text { SNDS } \\
\text { SHLE } \\
\text { EVPR } \\
\text { SHLE } \\
\text { ANDR } \\
\text { DINT } \\
\text { LMSN } \\
\text { SHLE } \\
\text { SNDS } \\
\text { SHLE } \\
\text { SNDS } \\
\text { LMSN } \\
\text { SHLE } \\
\text { SHLE } \\
\text { SNDS } \\
\text { LWSH } \\
\text { LMSH } \\
\text { LMSN } \\
\text { SNDS } \\
\text { LMSN } \\
\text { LMSN } \\
\text { DINT } \\
\text { LMSN } \\
\text { SHLB } \\
\text { DLYT }\end{array}$ \\
\hline
\end{tabular}


Table 3.--Geologic logs for wells in the study area--Continued

Oldham County, Texas

Lat1tude: 353500

Owner: SHELL Use of stte: $T$ Alt1tude: $\quad 3,496$
Longitude: 1021200

Local number: G M;BLK 2;SEC 64 Completion date: $02 / 16 / 1960$

Depth of well: 12,964

\begin{tabular}{|c|c|c|c|}
\hline $\begin{array}{l}\text { Geologic- } \\
\text { unit code }\end{array}$ & Depth to & $\begin{array}{l}\text { Depth to } \\
\text { bottom }\end{array}$ & L1thology \\
\hline 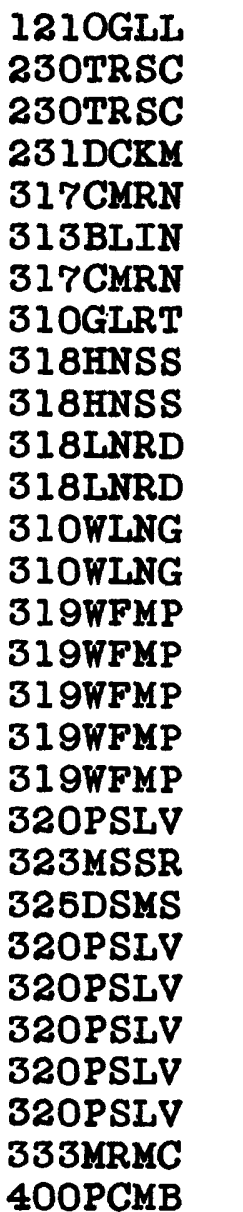 & $\begin{array}{r}85 \\
125 \\
540 \\
810 \\
923 \\
1,397 \\
1,650 \\
1,690 \\
1,740 \\
2,060 \\
2,290 \\
3,100 \\
3,690 \\
3,890 \\
4,093 \\
4,330 \\
5,400 \\
6,230 \\
6,444 \\
6,644 \\
7,760 \\
8,348 \\
8,990 \\
10,075 \\
11,620 \\
11,960 \\
12,348 \\
12,710 \\
12,920\end{array}$ & $\begin{array}{r}125 \\
540 \\
810 \\
923 \\
1,397 \\
1,650 \\
1,690 \\
1,740 \\
2,060 \\
2,290 \\
3,100 \\
3,690 \\
3,890 \\
4,093 \\
4,330 \\
5,400 \\
6,230 \\
6,444 \\
6,644 \\
7,760 \\
8,348 \\
8,990 \\
10,075 \\
11,620 \\
11,960 \\
12,348 \\
12,710 \\
12,920\end{array}$ & $\begin{array}{l}\text { SDGL } \\
\text { SDSL } \\
\text { SHLE } \\
\text { SNDS } \\
\text { SHLE } \\
\text { ANDR } \\
\text { DLMT } \\
\text { SNDS } \\
\text { BVPR } \\
\text { SDSL } \\
\text { BVPR } \\
\text { SHLE } \\
\text { ANDR } \\
\text { DLMT } \\
\text { DLMT } \\
\text { IMSN } \\
\text { LMSN } \\
\text { DLMT } \\
\text { SDSL } \\
\text { ARKS } \\
\text { SDSL } \\
\text { LMSN } \\
\text { SNDS } \\
\text { ARKS } \\
\text { LMSH } \\
\text { SHLE } \\
\text { LMSH } \\
\text { LMSN } \\
\text { IGNS }\end{array}$ \\
\hline
\end{tabular}


Table 3.--Geologic logs for wells in the study area--Continued

Oldham County, Texas

Latitude: 352315

Owner: SUPBRIOR OIL

Use of site: $P$

Alt1tude: $\quad 3,861$
Iong1tude: 1024240

Local number: LGE 31 CAPITAL LANDS

Completion date:

Depth of well: 5,499

\begin{tabular}{lrc|c}
$\begin{array}{l}\text { Geologio- } \\
\text { unit code }\end{array}$ & $\begin{array}{r}\text { Depth to } \\
\text { top }\end{array}$ & $\begin{array}{c}\text { Depth to } \\
\text { bottom }\end{array}$ & L1thology \\
313BLIN & 750 & 1,390 & ANDR \\
318CLFK & 1,390 & 2,820 & ANDR \\
318WCHT & 2,820 & 3,365 & SDSL \\
318WCHT & 3,365 & 3,670 & LMSH \\
319WFMP & 3,670 & 4,820 & LMSH \\
321CSCO & 4,820 & 5,460 & SHLE \\
400GRNT & 5,460 & & GRNT
\end{tabular}

\section{Oldham County, Texas}

Lat1tude: 351130

Owner: LIVERMORE IN

Use of site: $P$

Altitude: $\quad 3,881$
Long1tude: 1025715

Local number: 7N-1R, SBC. 26

Completion date:

Depth of well: 6,884

\begin{tabular}{|c|c|c|c|}
\hline $\begin{array}{l}\text { Geologio- } \\
\text { unit code }\end{array}$ & $\begin{array}{c}\text { Depth to } \\
\text { top }\end{array}$ & $\begin{array}{l}\text { Depth to } \\
\text { bottom }\end{array}$ & Lithology \\
\hline $\begin{array}{l}\text { 313BLIN } \\
318 \mathrm{CLFK} \\
318 \mathrm{WHT} \\
318 \mathrm{WHT} \\
319 \mathrm{WFMP} \\
320 \mathrm{PSLV} \\
\text { 400GRNT }\end{array}$ & $\begin{array}{l}2,170 \\
2,680 \\
3,290 \\
4,375 \\
4,680 \\
5,780 \\
6,740\end{array}$ & $\begin{array}{l}2,680 \\
3,290 \\
4,375 \\
4,680 \\
5,780 \\
6,740\end{array}$ & $\begin{array}{l}\text { ANDR } \\
\text { SHLB } \\
\text { SDSL } \\
\text { SHLB } \\
\text { DLMT } \\
\text { ARKS } \\
\text { GRNT }\end{array}$ \\
\hline
\end{tabular}


Table 3.--Geologic logs for wells in the study area--Continued

Potter County, Texas

Latitude: 352100

Owner: VHITTENBURG

Use of site: T

Altitude: $\quad 3,568$
Long1tude: 1014030

Local number: BS F;BLK 1; SEC 28 Complet1on date : 01/30/1955

Depth of well: 12,681

\begin{tabular}{|c|c|c|c|}
\hline $\begin{array}{l}\text { Geologic- } \\
\text { unit code }\end{array}$ & $\begin{array}{c}\text { Depth to } \\
\text { top }\end{array}$ & $\begin{array}{l}\text { Depth to } \\
\text { bottom }\end{array}$ & Litholog] \\
\hline 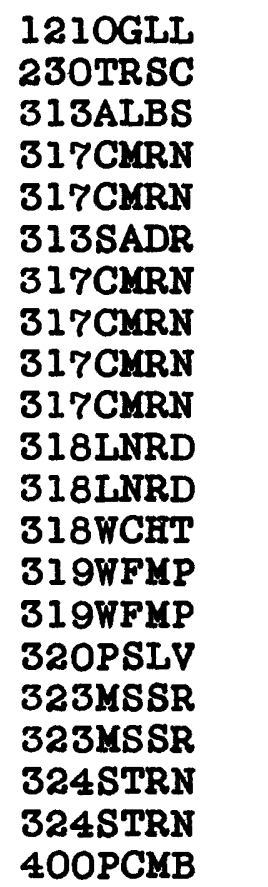 & $\begin{array}{r}30 \\
338 \\
550 \\
600 \\
895 \\
1,095 \\
1,770 \\
1,800 \\
2,703 \\
2,790 \\
3,330 \\
3,435 \\
3,665 \\
4,280 \\
5,596 \\
6,100 \\
7,520 \\
7,690 \\
7,850 \\
8,600 \\
8,740\end{array}$ & $\begin{array}{r}338 \\
550 \\
600 \\
895 \\
1,095 \\
1,770 \\
1,800 \\
2,703 \\
2,790 \\
3,330 \\
3,435 \\
3,665 \\
4,280 \\
5,596 \\
6,100 \\
7,520 \\
7,690 \\
7,850 \\
8,600 \\
8,740\end{array}$ & $\begin{array}{l}\text { SDGL } \\
\text { SHLE } \\
\text { DLMT } \\
\text { SDSL } \\
\text { ANDR } \\
\text { EVPR } \\
\text { SNDS } \\
\text { EVPR } \\
\text { SNDS } \\
\text { EVPR } \\
\text { SHLE } \\
\text { ANDR } \\
\text { DLMT } \\
\text { DLMT } \\
\text { LMSH } \\
\text { SDSL } \\
\text { LMSH } \\
\text { LMSN } \\
\text { LMSH } \\
\text { ARKS } \\
\text { GRNT }\end{array}$ \\
\hline
\end{tabular}


Table 3.--Geologic logs for wells in the study area--Continued

\section{Roberts County, Texas}

Latitude: 354130

Owner: PENNZOIL Use of site: $T$ Altitude: $\quad 2,795$
Long itude : 1003730

Locel number: H GN;BLK M2;SBC 62 Completion dete: $08 / 29 / 1974$

Depth of well: 14,552

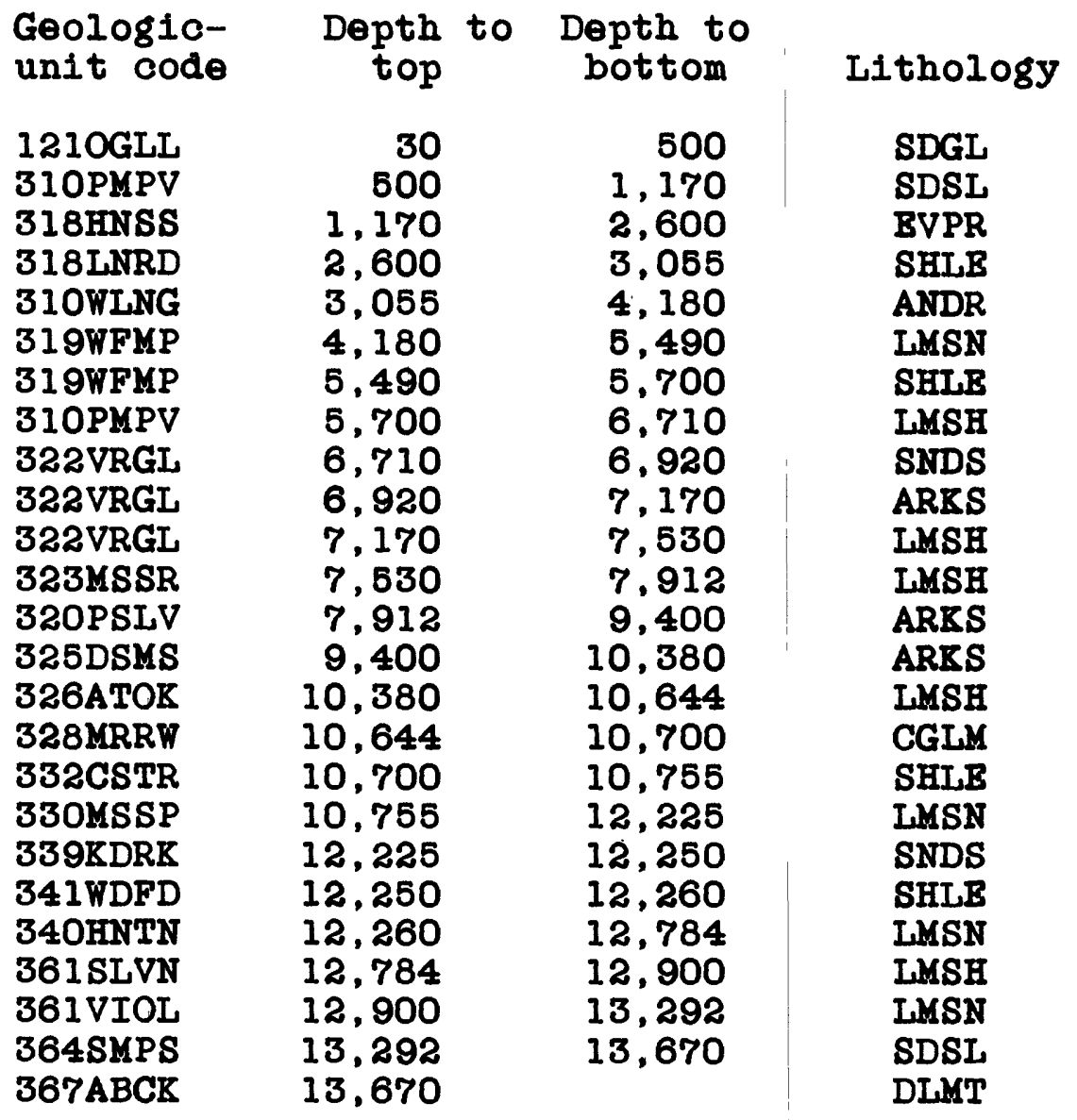


Table 3.--Geologic logs for wells in the study area--Continued

Sherman County, Texas

Lat1tude: 362230

Owner: PHILLIPS

Use of site: T

Altitude: $\quad 3,448$
Long1tude: 1014430

Local number: GH H;BLK 1C, SEC 68 Completion date: $01 / 13 / 1960$

Depth of well: 8,210

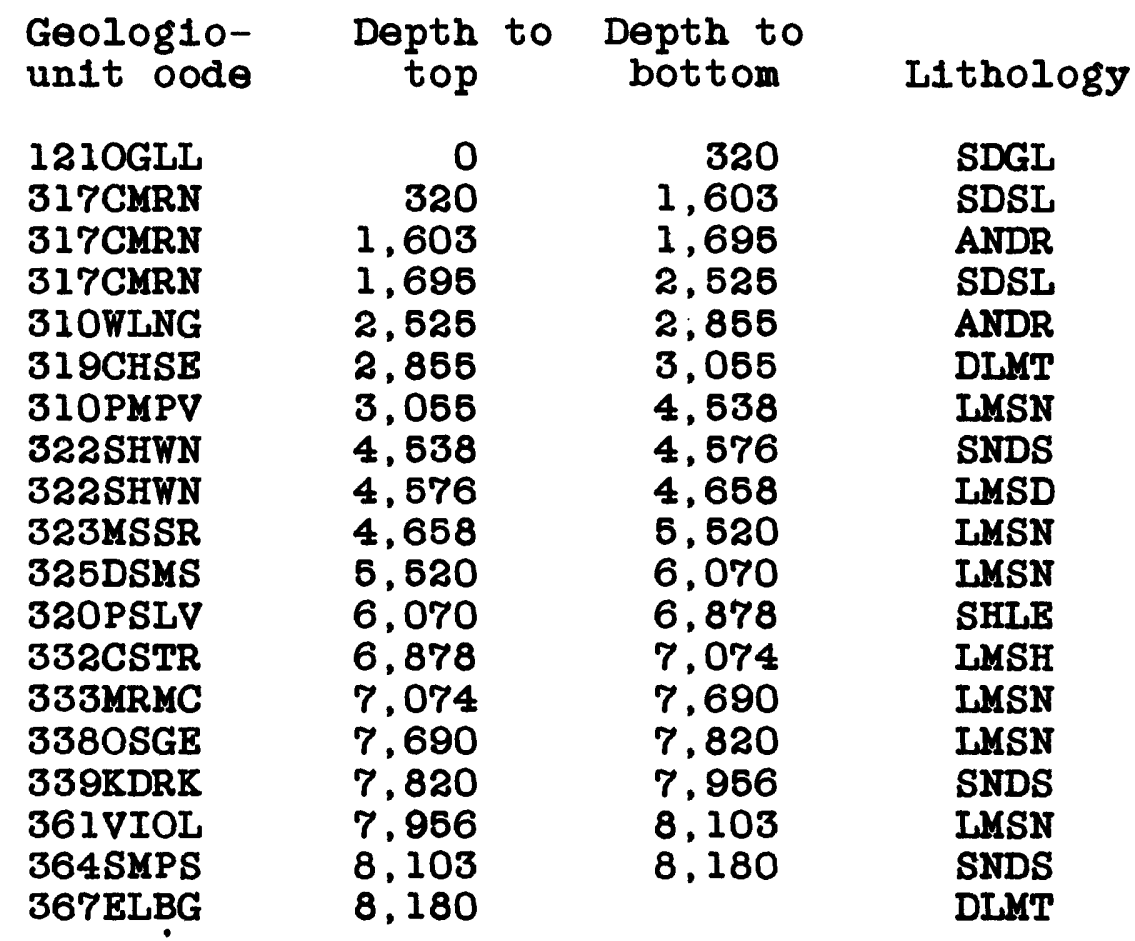

Sherman County, Texas

Lat1tude: 360700

Owner: I.T.I.O.

Use of site: $P$

Altitude: $\quad 3,639$
Long1tude: 1020130

Local number: T NO RR, IT, 369

Completion date:

Depth of well: B,138

$\begin{array}{lccc}\begin{array}{c}\text { Geolog10- } \\ \text { un1t code }\end{array} & \begin{array}{r}\text { Depth } \\ \text { top }\end{array} & \begin{array}{c}\text { Depth to } \\ \text { bottom }\end{array} & \text { L1thology } \\ \text { 318WCHT } & 1,400 & 2,175 & \text { SDSL } \\ \text { 318WCHT } & 2,175 & 2,240 & \text { DLMT } \\ \text { 319WFMP } & 2,240 & 2,800 & \text { DMSH } \\ \text { 321CSCO } & 2,800 & 3,440 & \text { LMSH } \\ \text { 321CNYN } & 3,440 & 3,900 & \text { IMSD } \\ \text { 330MSSP } & 3,900 & 4,350 & \text { LMSH } \\ \text { 364SMPS } & 4,360 & 4,460 & \text { SDSL } \\ \text { 367ELBG } & 4,460 & 5,120 & \text { DLMT } \\ \text { 400GRNT } & 5,120 & & \text { GRNT }\end{array}$


Table 3.--Geologic logs for wells in the study area--Continued

\section{Wheeler County, Texas}

Lat1tude: 362100 Owner: STD TEXAS Use of site: $T$ Altitude: $\quad 2,197$
Long1tude : 1000500

Local number: H GN;BLK A7, SEC 36 Completion date: 10/02/1970

Depth of well: 21,648
Geolog10unit code

$\begin{array}{lr}\text { 318LNRD } & 385 \\ \text { 318LNRD } & 550 \\ \text { 318LNRD } & 1,485 \\ \text { 318LNRD } & 2,610 \\ \text { 31OWLNG } & 3,055 \\ \text { 319WFMP } & 4,335 \\ \text { 319WFMP } & 6,145 \\ \text { 31OPMPV } & 6,220 \\ \text { 322DGLS } & 9,297 \\ \text { 322DGLS } & 9,440 \\ \text { 320PSLV } & 10,780 \\ \text { 320PSLV } & 12,660 \\ \text { 320PSLV } & 14,000 \\ \text { 320PSLV } & 16,060 \\ \text { 320PSLV } & 16,610 \\ \text { 330MSSP } & 17,272 \\ \text { 338OSGE } & 18,650 \\ \text { 341WDFD } & 20,470 \\ \text { 340FNTN } & 20,630 \\ \text { 361SLVN } & 21,606\end{array}$

Depth to
top

550

1,485

2,610

3,055

4,335

6,145

6,220

9,297

9,440

10,780

12,560

14,000

15,060

15,610

17,274

18,650

20,470

20,530

21,605
Lithology

ANDR

EVPR

SHLE

SHLB

ANDR

IMSN

SNDS

SDSL

SDSL

SHLB

SDSL

ARKS

IMSH

ARKS

IMSH

IMSSN

IMSNN

SHLB

IMDM

SHLE 
Table 3.--Geologic logs for wells in the study area--Cont Inued

Union County, New Mexico

Latitude: 360140

Owner: OIL EXPLORAT Use of site: P Altitude: $\quad 4,626$
Longitude: 1030746

Looal number: $21 \mathrm{~N}-36 \mathrm{E}-29$ BB

Completion date: $01 / 31 / 1966$

Depth of well: 7,360

$\begin{array}{lrcc}\begin{array}{l}\text { Geologio- } \\ \text { unit oode }\end{array} & \begin{array}{r}\text { Depth } \\ \text { top }\end{array} & \begin{array}{c}\text { Depth to } \\ \text { bottom }\end{array} & \text { L1thology } \\ \text { 211DKOT } & 130 & 280 & \text { SNDS } \\ \text { 217KION } & 280 & 298 & \text { SHLE } \\ \text { 217CYNN } & 298 & 345 & \text { SNDS } \\ \text { 221MRSN } & 345 & 525 & \text { SDSL } \\ \text { 221ENRD } & 525 & 565 & \text { SNDS } \\ \text { 231DCKM } & 655 & 1,010 & \text { SDSL } \\ \text { 231SNRS } & 1,010 & 1,625 & \text { SDSL } \\ \text { 310PRMN } & 1,525 & 1,847 & \text { DLNT } \\ \text { 313BLIN } & 1,847 & 2,112 & \text { ANDR } \\ \text { 310GLRT } & 2,112 & 2,173 & \text { SNDS } \\ \text { 318HNSS } & 2,173 & 2,687 & \text { SNDS } \\ \text { 317CMRN } & 2,687 & 3,046 & \text { SNDS } \\ \text { 310SGRC } & 3,045 & 7,268 & \text { ARKS } \\ \text { 367BLBG } & 7,268 & 7,297 & \text { DLMT } \\ \text { 371REGN } & 7,297 & 7,320 & \text { SNDS } \\ \text { 400PCMB } & 7,320 & & \text { GRNT }\end{array}$


Table 3.--Geologic logs for wells in the study area--Continued

Onion County, New Mexico

Latitude: 362015

Owner: CONTINENTAL

Use of site: P

Altitude: $\quad 4,678$
Long1tude: 1030320

Local number: $24 \mathrm{~N}-36 \mathrm{E}-2-\mathrm{DD}$

Completion date: 04/04/1957

Depth of well: 5,308

$\begin{array}{lr}\begin{array}{l}\text { Geolog10- } \\ \text { unit code }\end{array} & \begin{array}{r}\text { Depth } \\ \text { top }\end{array} \\ & \\ \text { 217CYNN } & 110 \\ \text { 221MRSN } & 238 \\ \text { 221ENRD } & 600 \\ \text { 231CHNL } & 650 \\ \text { 310PRMN } & 1,305 \\ \text { 310PRMN } & 1,340 \\ \text { 313BLIN } & 1,660 \\ \text { 310GLRT } & 2,043 \\ \text { 318HNSS } & 2,108 \\ \text { 317CMRN } & 2,492 \\ \text { 318WCHT } & 2,530 \\ \text { 310SGRC } & 2,685 \\ \text { 330MSSP } & 5,015 \\ \text { 367ELBG } & 5,180 \\ \text { 400PCMB } & 5,265\end{array}$

Depth to bottom

238

600

650

1,305

1,340

1,660

2,043

2,108

2,492

2,530

2,685

5,015

5,180

5,265

\section{Lithology}

SNDS

SDSL

SNDS

SDSL

SDSL

DIMT

ANDR

SNDS

ANDR

ANDR

SDSL

ARKS

IMSN

DINT

GRNT 
Table 3.--Geologic logs for wells in the study area--Continued

Union County, New Mexico

Latitude: 362745

Owner: HOXSEY OIL C

Use of site: P

Altitude: $\quad 5,509$
Long1tude: 1033030

Local number: 26N-32E-27 AD

Completion date: 08/01/1956

Depth of well: 3,898

$\begin{array}{lrcc}\begin{array}{l}\text { Geologic- } \\ \text { unit code }\end{array} & \begin{array}{r}\text { Depth } \\ \text { top }\end{array} & \begin{array}{c}\text { Depth to } \\ \text { bottom }\end{array} & \text { L1thology } \\ \text { 221MRSN } & 290 & 465 & \text { SDSL } \\ \text { 221ENRD } & 465 & 503 & \text { SNDS } \\ \text { 231DCKM } & 503 & 1,380 & \text { SDSL } \\ \text { 31OPRMN } & 1,380 & 1,942 & \text { DLMT } \\ \text { 313BLIN } & 1,942 & 2,125 & \text { ANDR } \\ \text { 31OGLRT } & 2,125 & 2,245 & \text { SDSL } \\ \text { 318HNSS } & 2,245 & 2,570 & \text { SNDS } \\ \text { 317CMRN } & 2,570 & 2,640 & \text { ANDR } \\ \text { 310SGRC } & 2,640 & 3,800 & \text { ARRS }\end{array}$

Union County, New Mexico

Latitude: 364438

Owner: TEXACO 1 CRU Use of site: $T$

Altitude: $\quad 4,835$
Long1tude: 1030408

Local number: 29N-36E-14 CCA 1

Completion date: 02/05/1964

Depth of well: 6,460

$\begin{array}{lrcc}\begin{array}{c}\text { Geologic- } \\ \text { unit code }\end{array} & \begin{array}{r}\text { Depth } \\ \text { top }\end{array} & \begin{array}{c}\text { Depth to } \\ \text { bottom }\end{array} & \text { L1thology } \\ \text { 211DKOT } & 20 & 266 & \text { SDSL } \\ \text { 220JRSC } & 266 & 465 & \text { SDSL } \\ \text { 220JRSC } & 465 & 550 & \text { LMSH } \\ \text { 230TRSC } & 550 & 1,422 & \text { SDSL } \\ \text { 310PRMN } & 1,422 & 1,448 & \text { DLMT } \\ \text { 317CMRN } & 1,448 & 1,690 & \text { SHLE } \\ \text { 313BLIN } & 1,690 & 1,940 & \text { GPSM } \\ \text { 310GLRT } & 1,940 & 1,970 & \text { SNDS } \\ \text { 318HNSS } & 1,970 & 2,505 & \text { EVPR } \\ \text { 319WFMP } & 2,505 & 3,715 & \text { SDSL } \\ \text { 320PSLV } & 3,715 & 5,600 & \text { ARKS } \\ \text { 330MSSP } & 5,600 & 6,015 & \text { IMDM } \\ \text { 367BLBG } & 6,015 & 6,422 & \text { DLMT } \\ \text { 400PCMB } & 6,422 & & \text { IGNS }\end{array}$


Table 3.--Geologic logs for wells in the study area--Continued

Union County, New Mexico

Lat1tude: 364710

Owner: NBLSON NOO

Use of site:

Altitude: $\quad 6,710$
Long1tude: 1035110

Local number: 30N-29B-33 DCC

Completion date: $07 / 25 / 1951$

Depth of well: 2,685

\begin{tabular}{lrr|r}
$\begin{array}{l}\text { Geologic- } \\
\text { un1t code }\end{array}$ & $\begin{array}{r}\text { Depth } \\
\text { top }\end{array}$ & $\begin{array}{c}\text { Depth to } \\
\text { bottom }\end{array}$ & Lithology \\
211DKOT & 0 & 81 & SNDS \\
217KIOW & 81 & 105 & SHLR \\
217CYNN & 105 & 145 & SNDS \\
221MRSN & 145 & 500 & SDSL \\
221TDLT & 500 & 535 & GPSM \\
221ENRD & 535 & 600 & SNDS \\
231CHNL & 600 & 1,055 & SDSL \\
310WTRS & 1,055 & 1,115 & SNDS \\
31OPRMN & 1,115 & 1,320 & DLMT \\
313BLIN & 1,320 & 1,378 & ANDR \\
310GLRT & 1,378 & 1,510 & SNDS \\
318HNSS & 1,610 & 1,798 & SNDS \\
310SGRC & 1,798 & 2,620 & ARKS \\
400PCKB & 2,620 & & GRNT
\end{tabular}

Union County, New Mexico

Latitude: 366546

Owner: GULF OIL CO

Use of site: P

Altitude: $\quad 6,300$
Longitude: 1032650

Local number: $31 \mathrm{~N}-33 \mathrm{~B}-17 \mathrm{BC}$

Completion date: $03 / 08 / 1970$

Depth of well: 2,866

\begin{tabular}{lrr|r}
$\begin{array}{l}\text { Geologic- } \\
\text { unit code }\end{array}$ & $\begin{array}{r}\text { Depth } \\
\text { top }\end{array}$ & $\begin{array}{r}\text { Depth to } \\
\text { bottom }\end{array}$ & Lithology \\
231CHNL & 330 & 760 & SDSL \\
231SNRS & 760 & 885 & SNDS \\
31OPRMN & 885 & 915 & DLMT \\
310WTRS & 916 & 1,067 & \\
313BLIN & 1,067 & 1,180 & \\
310GLRT & 1,180 & 1,280 & SNDS \\
310YBSO & 1,280 & 1,568 & \\
310SGRC & 1,558 & 2,538 & ARRS \\
330MSSP & 2,638 & 2,605 & LMSN \\
367BLBG & 2,605 & 2,756 & DLMT \\
400PCMB & 2,756 & & GRNT
\end{tabular}


Table 4.--Geophysical logs for wells in the study area

Adair County, Oklahoma

Latitude: 355931

Owner: WESTVILLE OK Use of site: $W$

Altitude: 1,123

Type of $\log$

Caliper

Electrio

Gamma

Neutron
Longitude: 943400

Local number: 18N-26E-31 DAD 1

Completion date: 08/07/1981

Depth of well: 1,500

Begin depth End depth

Adair County, Oklahoma

Latitude: 360507

Owner: ARTIE BROWN ONE

Use of site: W

Altitude: 1,070

Type of log

Drillers

$\begin{array}{rr}400 & 1,497 \\ 490 & 1,498 \\ 4 & 1,495 \\ 3 & 1,499\end{array}$

Longitude: 944022

Local number: 19N-25E-32 BDA 1 Completion date:

Depth of well: 1,430

Begin depth End depth

400

1,497

Alfalfa County, Oklahoma

Lat1tude: 363402

Owner: OLSON DRLG

Use of site: $T$

Altitude: 1,379

Type of $\mathrm{log}$

Electrio

Geologist
Longltude: 981901

Local number: 24N-11W-12 DDA 1

Completion date: 09/05/1942

Depth of well: 7,300

Begin depth End depth

100

40
7,300

7,300 
Table 4.--Geophysical logs for wells in the study area--Continued

Atoka County, Oklahoma

Lat1tude: 342016

Owner: LEWIS

Use of site: P

Alt1tude :

Type of 108

Geolog 1st

Induction

Microlog
599
1.Long1tude: 961130

Local number: 02S-11B-31 CA 1

Completion date: $03 / 16 / 1958$

Depth of well: 11,833
Begin depth

$$
\begin{array}{r}
50 \\
100 \\
9,200
\end{array}
$$

End depth
11,833
11,828
11,833

Atoka County, Oklahoma

Lat1tude: 341450

Owner: CROSSLAND

Use of site: P

Altitude :

Type of $10 \mathrm{~g}$

Caliper

Induct ion

Gamma

Sonic
512
Long1tude: 961304

Local number: 03S-10B-35 DD 1

Completion date: $10 / 08 / 1973$

Depth of well: 8,125

Begin depth Bnd depth

$\begin{array}{rr}550 & 8,124 \\ 604 & 8,122 \\ 0 & 8,124 \\ 603 & 8,124\end{array}$

Beaver County, Oklahoma

Lat1tude: 365335

Owner: PURE OIL

Use of site: $T$

Alt1tude: $\quad 2,597$

Type of 108

Blectric

Geologist
Long1tude: 1001507

Local number: 05N-26BCM-16 CDD 1

Completion date: 12/30/1951

Depth of well: $\quad 9,240$

Begin depth

Bnd depth

270

9,228

0

9,240 
Table 4.--Geophysical logs for wells in the study area--Continued

Beckham County, Oklahoma

Latitude: 351225

Owner: CARTER

Use of site: $P$

Altitude: $\quad 1,799$

Type of $\log$

Electric

Geologist
Longitude: 992823

Local number: 09N-22W-36 DDB 1 Completion date: 05/22/1951

Depth of well: 3,100

$$
\text { Begin depth End depth }
$$

3,090

\section{Beckham County, Oklahoma}

Latitude: 351835

Owner: LONE STAR

Use of site: P

Altitude: 1,953

Type of $\log$

Caliper

Electric

Geolog 1st

Gamma

Neutron

Sonic

Gamma-gamma

Other
Long1tude: 993142

Local number: 10N-22W-28 DCA 1 Completion date: 10/20/1972

Depth of well: 30,000
Begin depth

$$
\begin{array}{r}
4,822 \\
4,822 \\
5,160 \\
0 \\
4,822 \\
4,822 \\
4,823 \\
23,390
\end{array}
$$

End depth

30,000
30,000
30,000
30,000
22,726
30,000
29,978
30,000

Beckham County, Oklahoma

Latitude: 351816

Owner: CARTER

Use of site: P

Altitude: 1,828

Type of 108

Electric

Geologist
Longitude: 993453

Local number: 10N-23W-36 ABA 1

Completion date: 09/28/1950

Depth of well: 10,654

$$
\text { Begin depth End depth }
$$

86

10,648

0

10,654 
Table 4.--Geophysical logs for wells in the study area--Continued

Bla1ne County, Oklahoma

Lat1tude: 360746

Owner: SUPERIOR

Use of site: P

Altitude: 1,200

Type of 108

Electr10

Geologist
Long1 tude: 981658

Local number: 19N-10W-17 AAA 1

Completion date: 01/01/1945

Depth of well: 11,008

Begin depth End depth

$25 \quad 11,008$

$0 \quad 11,008$

Caddo County, Oklahoma

Lat1tude: 361119

Owner: SUPERIOR

Use of stte: P

Altitude: 1,440

Type of 108

Eleotrio

Geologist
Long1tude: 982556

Local number: 08N-12W-11 ABB 1

Complet1on date: 01/01/1947

Depth of well: 17,823

Begin depth End depth

1,539

17,738

140

Canadian County, Oklahoma

Lat1tude: 362521

Owner: PETREE

Use of site: $P$

Alt1tude: $\quad 1,310$

Type of 108

Electrio

Geologist
Longltude: 980460

Local number: 11N-08W-18 DD 1 Completion date:

Depth of well: 11,177

Begin depth End depth

365

450
11,170

11,177

Canadian County, Oklahoma

Lat1tude: 353053

Owner: HUCHTEMAN

Use of site: P

Altitude: $\quad 1,344$

Type of 108

Electric

Geologist

Miorolog
Longitude: 976418

Local number: $12 N-07 W-14$ DAB 1 Completion date:

Depth of well: 11,700
Begin depth End depth

$\begin{array}{rr}417 & 11,500 \\ 620 & 11,700 \\ 7,260 & 11,600\end{array}$


Table 4.--Geophysical logs for wells in the study area--Continued

Carter County, Oklahoma

Lat1tude: 342921

Owner: MEEKS

Use of site: P Altitude :

Type of 108

Geolog1st

Induation

Gamma

Miorolog

Neutron

Gamma-gamma
938
Long1tude: 972402

Local number: 01S-02W-10 BBD 1 Completion date: 03/22/1966

Depth of well: 3,018

Begin depth End depth

$\begin{array}{rr}315 & 3,000 \\ 317 & 3,017 \\ 10 & 316 \\ 316 & 3,015 \\ 10 & 316 \\ 316 & 3,017\end{array}$

Carter County, Oklahoma

Lat1tude: 341618

Owner: WHITE EAGLE

Use of site: P

Altitude :

763
Long1tude: 971010

Local number: 03S-01E-26 BAD 1

Completion date: 10/31/1977

Depth of well: 16,950

Type of 108

Begin depth

End depth

Geologist

Laterolog

Neutron

Son10

Cherokee County, Oklahoma

Lat1tude: 354856

Owner: PINE

Use of site: $T$

Altitude :

690
6,400
4,088
6,946
4,088
16,925

16,122

16,918

16,122

Type of $10 \mathrm{~g}$

Begin depth

Bnd depth

Geolog1st

62

2,093

Long1tude: 950444

Looal number: $16 \mathrm{~N}-21 \mathrm{E}-33 \mathrm{DCA} 1$

Completion date: 01/01/1962

Depth of well: 2,093 
Table 4.--Geophysical logs for wells in the study area--Continued

Cherokee County, Oklahoma

Lat1tude: 360502

Owner: M F OIL CO

Use of site: T

Altitude:

800
Long1tude: 950152

Local number: 19N-21E-35 AD 1

Completion date: 01/01/1961

Depth of well: 1,935

Type of 108

Begin depth

Bnd depth

Geolog1st 460 1,935

Lat1 tude: 365818

Owner: SUN CO.

Use of site: T

Altitude: $\quad 4,305$

Type of 108

Eleotrio

Geologist
Long1tude: 1024134

Local number: 06N-03B-24 CB 1

Completion date: 06/24/1954

Depth of well: 5,904

Begin depth End depth

$0 \quad 5,904$

$0 \quad 5,904$

Cleveland County, Oklahoma

Lat1tude: 351432

Owner: ROSB

Use of site: P

Altitude: 1,127

Type of 108

Eleotrio

Geologist

Miorolog
Long1tude: 973138

Local number: 09N-03W-21 BCA 1 Completion date:

Depth of well: 10,590

Begin depth Bnd depth

$\begin{array}{ll}1,002 & 10,588 \\ 3,000 & 10,590 \\ 8,050 & 10,588\end{array}$

Coal County, Oklahoma

Lat1tude: 343349

Owner: CRIM

Use of site: $P$

Altitude:

674
Long1tude: 960953

Loo I number: O1N-11E-17 ABA 1 Complet1on date: $07 / 14 / 1972$

Depth of well: 11,632

Type of 108

Begin depth

Bnd depth

Induotion

Gamma

70

70

1,550

11,632

1,257

11,632 
Table 4.--Geophysical logs for wells in the study area-Continued

Coal County, Oklahoma

Latitude: 344000

Owner: MORGAN

Use of site: $P$

Altitude :

725
Longitude: 962036

Local number: 02N-09E-03 DC 1

Completion date: 09/13/1968

Depth of well: 8,038

Type of 108

Begin depth

Bnd depth

Geologist

Induction

Gamma-gamma

8,038

8,038

8,038

Comanche County, Oklahoma

Latitude: 344646

Owner: HUMBLE OIL

Use of site: $P$

Altitude: 1,210

Type of 108

Blectric

Geologist
Longitude: 982218

Local number: 03N-11W-OB AD 1

Completion date: 10/16/1964

Depth of well: 1,691

Begin depth Bnd depth

253

1,688

$0 \quad 1,591$

Comanche County, Oklahoma

Latitude: 344626

Owner :

Use of site: $P$

Altitude: 1,272

Type of 108

Caliper

Electric

Geologist

Gamma

Sonic
Longitude: 981746

Local number: 04N-10W-31 CBA I Completion date: $11 / 11 / 1968$

Depth of well: 6,603

Begin depth End depth

1,208

6,591

70

6,594

60

6,603

1,208

6,691

1,420

6,691 
Table 4.--Geophysical logs for wells in the study area--Continued

Craig County, Oklahoma

Latitude: 365016

Owner: NEILI

Use of site: W

Altitude :

Type of 108

Geologist
Long1tude: 950705

Local number: $27 \mathrm{~N}-20 \mathrm{~B}-12$ BDD 2 Completion date: 06/13/1971

Depth of well: 1,090

Begin depth End depth

550

1,090

Craig County, Oklahoma

Latitude: 365000

Owner: RWD-3

Use of site: W

Altitude :

Type of 108

Caliper

Electric

Fluid

Gamma

876
Long1tude: 950101

Local number: $27 \mathrm{~N}-21 \mathrm{E}-12$ CCB 1

Completion date : 07/07/1980

Depth of well: 1,352
Begin depth

687

200

185

4
End depth

1,350

1,352

1,350

1,352

Creek County, Oklahoma

Latitude: 355718

Owner: BERRYHILL

Use of site: P

Altitude :

Type of 108

Electric

Microlog

710
Long1tude: 960308

Local number: 17N-12E-17 ACA 1 Completion date:

Depth of well: 3,932

Begin depth End depth

\begin{tabular}{l|l}
280 & 3,930 \\
660 & 3,928
\end{tabular}

Custer County, Oklahoma

Latitude: 354318

Owner: ARKLA

Use of site: $P$

Altitude: 1,795

Type of $\log$

Geologist
Long1tude: 990029

Local number: 14N-17W-04 A 1 Completion date: 05/12/1972

Depth of well: 16,700

Begin depth End depth

600 
Table 4.--Geophysical logs for wells in the study area--Continued

Custer County, OkIahoma

Lat1tude : 354548

Owner: MACNOLIA

Use of site: P

Alt1tude: 1,794

Type of $10 \mathrm{~g}$

Eleotric

Geologist

Sonic
Longltude: 985328

Local number: $15 \mathrm{~N}-16 \mathrm{~W}-22$ BD 1

Completion date: 04/07/1959

Depth of well: 17,000

Begin depth Bnd depth

$\begin{array}{rr}269 & 16,996 \\ 10,800 & 17,000 \\ 11,479 & 16,993\end{array}$

Delaware County, Oklahoma

Latitude: 362442

Owner: RWD-1

Use of site: W

Alt1tude: 1,100

Type of $10 \mathrm{~g}$

Geologist
Longltude: 945208

Local number: 22N-23B-05 DCA 1

Completion date: 06/18/1979

Depth of well: 1,538

Begin depth Fnd depth

Dewey County, Oklahoma

Lat1tude: 360821

Owner: MAGNOLIA

Use of site: $P$

Altitude: $\quad 2,225$

Type of 108

Caliper

Eleotrio

Geologist

Gemma

Neutron
Longltude: 992007

Local number: $19 \mathrm{~N}-20 \mathrm{~W}-09$ AC 1

Completion date: 09/28/1947

Depth of well: 12,856

Begin depth

End depth

6,700

332

310

6,700

6,700
12,748

13,800

13,811

12,748

12,748 
Table 4.--Geophysical logs for wells in the study area-Continued

Blils County, Oklahoma

Latitude: 355912

Owner: MCCULIOCH

Use of site: T

Altitude: $\quad 2,395$

Type of $\log$

Caliper

Geologist

Induction

Gamma.

Laterolog

Neutron

Sonic

Other
Longitude: 994508

Local number: $18 \mathrm{~N}-24 \mathrm{~W}-34$ CCA 1

Completion date: 03/12/1971

Depth of well: 17,500

Begin depth Bnd depth

$\begin{array}{rr}11,800 & 16,419 \\ 7,000 & 17,500 \\ 4,370 & 16,419 \\ 16,250 & 17,500 \\ 11,800 & 16,419 \\ 16,250 & 17,500 \\ 11,770 & 16,415 \\ 11,771 & 16,419\end{array}$

Bllis County, Oklahoma

Latitude: 363140

Owner: GULF OIL Use of site: $T$

Altitude:

2,382
Longitude: 995830

Local number: 24N-26W-27 CA 1 Completion date: 05/15/1944

Depth of well: $\quad 9,305$

Type of 108

Begin depth

End depth

Electric

Geologist

9,298

9,305

Garfield County, Oklahoma

Latitude: 362951

Owner: VOLIN

Use of site: P

Altitude: 1,050

Type of 108

Geologist

Neutron

Gamma-gamma
Long1tude: 973446

Local number: 23N-04W-01 CAA 1 Completion date:

Depth of well: 7,152
Begin depth

2,400

5,200

1,070
End depth

7,100

7,150

7,150 
Table 4.--Geophysical logs for wells in the study area--Continued

Garvin County, Oklahoma

Latitude: 343139

Owner: HUMBLE OIL Use of site: P Altitude:

Type of $\log$

Caliper

Geologist

Gamma

Dip meter

Sonic
857

Begin depth
Long1tude: 972018

Local number: 01N-01W-30 DAB 1 Completion date: 06/24/1971

Depth of well: 13,187

Grady County, Oklahoma

Latitude: 344547

Owner: PARKER

Use of site: $P$

Altitude: 1,123

Type of $\log$

Electric

Geologist

Gamma

Sonic
Longitude: 974029

Local number: 03N-05W-01 AC 1

Completion date: 11/01/1966

Depth of well: 18,413

Begin depth End depth

$\begin{array}{rr}2,490 & 18,382 \\ 6,600 & 18,413 \\ 15,814 & 18,382 \\ 15,814 & 18,382\end{array}$

Grady County, Oklahoma

Latitude: 350345

Owner: PHILLIPS

Use of site: $P$

Altitude: 1,161

Type of 108

Caliper

Electric

Geologist

Gamma

Other
Longitude: 980307

Local number: 07N-08W-21 CAC 1

Completion date: 10/29/1969

Depth of well: 10,754

Begin depth

End depth
10,747

10,754

10,754

10,747

10,747 
Table 4.--Geophysical logs for wells in the study area-Continued

Grant County, Oklahoma

Latitude: 365939

Owner: HENDRIXON

Use of site: P

Altitude: 1,271

Type of $10 \mathrm{~g}$

Electric

Geologist
Longitude: 980513

Local number: 29N-08W-17 BDB 1

Completion date:

Depth of well: 5,660

Begin depth End depth

262

400
5,660

5,660

Harper County, Oklahoma

Latitude: 364014

Owner: CONTINENTAL

Use of site: T

Altitude: 1,924

Type of $\log$

Electric

Geologist
Longitude: 993209

Local number: $25 \mathrm{~N}-22 \mathrm{~W}-02$ DBD 1

Completion date: 09/10/1949

Depth of well: 9,440
Begin depth

321

0
End depth

9,440

9,436

Haskell County, Oklahoma

Latitude: 350957

Owner: GORDON

Use of site: P

Altitude :

Type of 108

Electric

Geologist 636
Longitude: 951138

Local number: 08N-20E-16 DBB 1

Completion date: 02/03/1955

Depth of well: 6.077
Begin depth

529

15
End depth

6,069

6,077

Haskell County, Oklahoma

Latitude: 351010

Owner: ALLRED

Use of site: $P$

Altitude:

685
Longitude: 951345

Local number: 08N-20E-18 ADB 1

Completion date: 08/28/1951

Depth of well: 7,483
Type of 108

Electric

Geologist

Miorolog
Begin depth

763

800

5,590
End depth

7,482

7,483

7,480 
Table 4.--Geophysical logs for wells in the study area--Continued

\section{Hughes County, Oklahoma}

Latitude: 345529

Owner: PIERCE

Use of site: P

Altitude:

825
Iong1tude : 961838

Local number: 05N-09E-12 BAD 1 Completion date:

Depth of well: 6,957
Type of 108

Electric

Geologist
Begin depth

100

700
End depth

6,557

6,957

\section{Johnston County, Oklahoma}

Latitude: 341328

Owner: CHAPMAN

Use of site: P

Altitude:

655
Long1tude: 964912

Local number: 04S-05E-07 BDD 1 Completion date: 04/15/1979 Depth of well: 11,747
Type of 108

Cal1per

Geologist

Induction

Neutron
Begin depth

1,660

70

1,660

1,660
End depth

11,705

11,747

11,705

11,705

Kay County, Oklahoma

Latitude: 365414

Owner: WELCH

Use of site: P

Alt1tude: 1,138

Type of 108

Electric

Microlog
Longltude: 971324

Local number: 28N-01E-17 CAB 1 Completion date:

Depth of well: 4,407

Begin depth End depth

$\begin{array}{rl}100 & 4,407 \\ 1,692 & 4,407\end{array}$


Table 4.--Geophysical logs for wells in the study area--Continued

\section{Kingfisher County, Oklahoma}

Latitude: 355619

Owner: ASH

Use of site: P

Altitude: 1,005

Type of $\log$

Electric

Geologist

Microlog
Longitude: 974613

Local number: 17N-05W-19 ABD 1 Completion date:

Depth of well: 8,519

Begin depth End depth

$\begin{array}{ll}1,024 & 8,518 \\ 4,000 & 8,515 \\ 6,081 & 7,282\end{array}$

Kingfisher County, Oklahoma

Latitude: 360132

Owner: VILHAUER

Use of site: P

Altitude: 1,188

Type of $\log$

Electric

Geologist
Longitude: 980903

Local number: 18N-09W-22 BAC 1 Completion date:

Depth of well: $\quad 9,639$
Begin depth End depth 255

0
9,639

9,639

Klowa County, Oklahoma

Latitude: 350539

Owner: EASON OIL Use of site: P Altitude: 1,689

Type of $\log$

Caliper

Geologist

Gamma.

Neutron
Longitude: 991400

Local number: 07N-19W-08 CBA 1 Completion date: 11/26/1976

Depth of well: 4,296

Begin depth End depth

407

400

407

407
4,296

4,290

4,296

4,296 
Table 4.--Geophysical logs for wells in the study area--Continued

Latimer County, Oklahoma

Latitude: 345706

Owner: FIELDS

Use of site: P

Altitude :

Type of $10 \mathrm{~g}$

Geologist

Induction

Gamma

Neutron

Gamma-gamma
Longitude: 950120

Local number: 06N-22E-31 BDB 1 Completion date: 04/08/1977

646
Begin depth End depth

$\begin{array}{rr}300 & 12,350 \\ 312 & 12,324 \\ 312 & 12,324 \\ 4,500 & 12,322 \\ 312 & 12,322\end{array}$

Leflore County, Oklahoma

Latitude: 351010

Owner: HICKMAN

Use of site: $P$

Altitude:

Type of 108

Electric

Geologist 465
Longitude: 944115

Local number: $08 \mathrm{~N}-25 \mathrm{E}-17$ BDB 1 Completion date: 01/29/1952

Depth of well: 7,650
Begin depth

400

0
End depth

6,916

7,650

Leflore County, Oklahoma

Latitude: 351128

Owner: COLEMAN

Use of site: $P$

Altitude : 419
Long1tude: 943503

Local number: 08N-26E-05 CCA 1 Completion date: $11 / 21 / 1974$ Depth of well: 8,841
Type of $\log$

Geologist

Induction

Microlog

Temperature

Gamma-gamma
Begin depth

7,400

602

6,100

502

5,750
End depth
8,841
8,827
7,646
8,834
8,826 
Table 4.--Geophysical logs for wells in the study area--Continued

Lincoln County, Oklahoma

Latitude: 353528

Owner: LINAN

Use of site: $P$

Altitude: 1,038

Type of $\log$

Electric

Geologist
Longitude: 970039

Local number: 13N-03E-20 BAC 1

Completion date:

Depth of well: 6,350

Begin depth End depth

264

6,341

3,000

6,340

Lincoln County, Oklahoma

Latitude: 354040

Owner: CUNNINGHAM

Use of site: $P$

Altitude:

933
Longitude: 965221

Local number: 14N-04B-22 BCA 1 Completion date:

Depth of well: 6,024

Type of 108

Begin depth

End depth

Electric

151

Geologist

190

5,023

5,024

Logan County, Oklahoma

Latitude: 354629

Owner: KROUT

Use of site: P

Altitude: $\quad 1,006$

Type of $\log$

Electric

Geologist

Gamma

Microlog
Longitude: 973554

Local number: 15N-04W-14 CBA 1 Completion date:

Depth of well: 8,350

Begin depth End depth
311
2,400
7,180
3,800

8,350

7,350

8,350

7,350 
Table 4.--Geophysical logs for we lls in the study area--Continued

McClain County, Oklahoma

Latitude: 345956

Owner: LITTLE

Use of site: $P$

Altitude: 1,134

Type of 108

Caliper

Electric

Geologist

Gamma.

Sonic
Long1tude: 972342

Local number: 06N-02W-15 AB 1 Completion date: 07/08/1971 Depth of well: 10,037
Begin depth

7,100

521

30

7,100

7,100
End depth

10,030

10,036

9,700

10,030

10,030

MoIntosh County, Oklahoma

Latitude: 351503

Owner: FOLLANSBEE

Use of site: $P$

Altitude:

Type of $\log$

Electric

Geologist

Microlog
653
Longitude: 954538

Local number: 09N-15E-18 CBC 1

Completion date: $11 / 28 / 1950$

Depth of well: 5,461

Begin depth End depth

$\begin{array}{rr}503 & 5,451 \\ 20 & 5,461 \\ 1,790 & 5,458\end{array}$

Major County, Oklahoma.

Latitude: 362139

Owner: SUPERIOR

Use of site: $P$

Altitude: 1,276

Type of $\log$

Electric

Geologist
Longitude: 981527

Local number: 22N-10W-27 BAA 1

Completion date: $01 / 01 / 1945$

Depth of well: 8,279

Begin depth

End depth

30

30
8,279

8,279 
Table 4.--Geophysical logs for wells in the study area--Continued

\section{Mayes County, Oklahoma}

Lat1tude: 360823

Owner: BALLOU

Use of site: $P$

Altitude:

Type of $\log$

Geologist
Longitude: 951111

Local number: 19N-20E-09 BCD 1 Completion date: 01/01/1967 Depth of well: 1,124
Begin depth

175
End depth

1,124

\section{Murray County, Oklahoma}

Latitude: 343046.

Owner: FLEMING

Use of site: T

Altitude:

Type of log

Induction

Microlog

Gamma-gamma

Geologist
Longitude: 970040

Local number: 01N-03E-32 CAA 1

Completion date: 06/04/1973

Depth of well: 5,178

Begin depth End depth

100

100

819

0
5,178

799

5,178

5,178

Muskogee County, Oklahoma

Latitude: 353510

Owner: MARSHALL

Use of site: P

Altitude:

601
Longitude: 951528

Local number: $13 \mathrm{~N}-19 \mathrm{E}-23 \mathrm{DB} 1$

Completion date: 01/09/1958

Depth of well: 3,296
Type of 108

Electric

Gamma

Microlog

Neutron
Begin depth

250

300

400

300
End depth

3,290

3,289

2,000

3,289 
Table 4.--Geophysical logs for wells in the study area--Continued

\section{Noble County, Oklahoma}

Latitude: 361218

Owner : HUBBART

Use of site: $P$

Altitude: 1,100

Type of $\log$

Electric

Geologist
Longitude: $\quad 972036$

Local number: 20N-01\%-18 CDB 1 Completion date:

Depth of well: 6,239

Begin depth End depth

$200 \quad 5,239$

$220 \quad 5,239$

Noble County, Oklahoma

Latitude: 361216

Owner: MAGNEY

Use of site: $P$

Altitude: 1,269

Type of $\log$

Electric

Geologist
Long1tude: $\quad 972626$

Local number: 20N-02W-17 CC 1 Completion date:

Depth of well: 7,088

Begin depth End depth

$\begin{array}{ll}5,062 & 7,085 \\ 6,274 & 7,088\end{array}$

Nowata County, Oklahoma

Latitude: 366100

Owner: ALSUP

Use of site: $P$

Altitude: $\quad 760$

Type of $\log$

Electric

Microlog
Longitude: $\quad 953920$

Local number: 27N-15E-01 DBB 1 Completion date: 06/07/1954

Depth of well: 2,222

Begin depth End depth

101

101

2,216

2,212

Okfuskee County, Oklahoma

Latitude: 353066

Owner: REPLOGLE

Use of site: $P$

Altitude:

Type of $\log$

Electric

Geologist
944
Longitude: 962714

Local number: 12N-08E-15 CAB 1

Completion date:

Depth of well: 4,507
Begin depth End depth

89

1,000
4,606

4,600 
Table 4.--Geophysical logs for wells in the study area-Continued

Oklahoma County, Oklahoma

Latitude: 352505

Owner: FARLEY

Use of site: $P$

Altitude: 1,242

Type of 108

Electric

Geologist
Longitude: 972712

Local number: 11N-02W-19 BAC 1

Completion date:

Depth of well: 8,342

Begin depth End depth

8,342

8,342

Oklahoma County, Oklahoma

Lat1tude: 353755

Owner : MACKLIN

Use of site: P

Altitude: 1,030

Type of $\log$

Electric

Geologist
Longitude: 971525

Local number: 13N-01W-01 BDD 1 Completion date:

Depth of well: 5,942

Begin depth End depth

$228 \quad 5,934$

350

5,942

Okmulgee County, Oklahoma

Latitude : 354922

Owner: MILLER

Use of site: P

Altitude:

802
Longltude : 955050

Local number: 16N-14E-32 BDA 1 Completion date:

Depth of well: 3,915
Type of 108

Electric
Begin depth

115
End depth

3,915

Osage County, Oklahoma

Latitude: 361940

Owner: ZINK

Use of site: $P$

Altitude :

Type of $\log$

Electrio

Miorolog

890
Long1tude: 960915

Local number: 21N-1IE-05 BC 1 Completion date:

Depth of well: 2,995
Begin depth End depth

80

1,000
2,990

2, 990 
Table 4.--Geophysical logs for wells in the study area-Continued

Osage County, Oklahoma

Latitude: 362015

Owner: SUNRAY

Use of site: $P$

Altitude:

836
Long1tude: 961313

Local number: $22 \mathrm{~N}-10 \mathrm{E}-34 \mathrm{C} \quad 1$

Completion date:

Depth of well: 3,235

Type of $\log$

Begin depth

End depth

Electric

327
2,300

3,234

Miorolog

3,232

Osage County, Oklahoma

Latitude: 363722

Owner: PHILLIPS

Use of site: P

Altitude:

Type of 108

Caliper

Eleotrio

Microlog
Longitude: 962512

Local number: 25N-08E-23 CCC 1

Completion date: 08/15/1956

Depth of well: 3,663

898

Begin depth

2,316
127
2,316

End depth

3,663

3,663

3,663

Ottawa County, Oklahoma

Latitude: 365324

Owner: GOODRICH RUB.CO

Use of site: W

Altitude: $\quad 798$

Type of $\log$

Geologist
Longitude: 945313

Local number: 28N-22E-24 CAD 1 Completion date: 01/01/1944

Depth of well: 1,065

Begin depth End depth

2,316

3,663

Pawnee County, Oklahoma

Latitude: 362551

Owner: TRIGG

Use of site: $P$

Altitude:

Type of 108

Eleotric

Geologist
Longitude: 965836

Local number: 23N-03E-34 BCB 1 Completion date:

Depth of well: 4,585

Begin depth End depth

147

4,375

1,500

4,375 
Table 4.--Geophysical logs for wells in the study area--Continued

Payne County, Oklahoma

Lat1tude: 361024

Owner: FLYNN

Use of site: P

Altitude:

960
Iong1tude: 970614

Local number: 20N-02E-33 BBB I Completion date:

Depth of well: 4,877

Type of $\log$

Begin depth

End depth

Electric

162

Goologist

200

4,877

4,877

P1ttsburg County, Oklahoma

Lat1tude: 345558

Owner: SEALY

Use of site: P

Altitude:

Type of 108

Eleotrio

Geologist
Long1tude: 955923

Local number: 05N-12E-01 CA 1

Completion date: 06/01/1964

Depth of well: 3,541

Begin depth End depth

175

400
3,536

3,541

Pittsburg County, Oklahoma

Latitude: 345758

Owner: MANSHRICK

Use of site: $P$

Altitude :

963
Longitude: 963023

Local number: 06N-17E-28 ADB 1

Completion date: 09/23/1954

Depth of well: 12,915
Type of $\mathrm{log}$

Electric

Geologist
Begin depth

68

3,325
End depth

12,914

12,915 
Table 4.--Geophysical logs for wells in the study area--Continued

\section{Pontotoc County, Oklahoma}

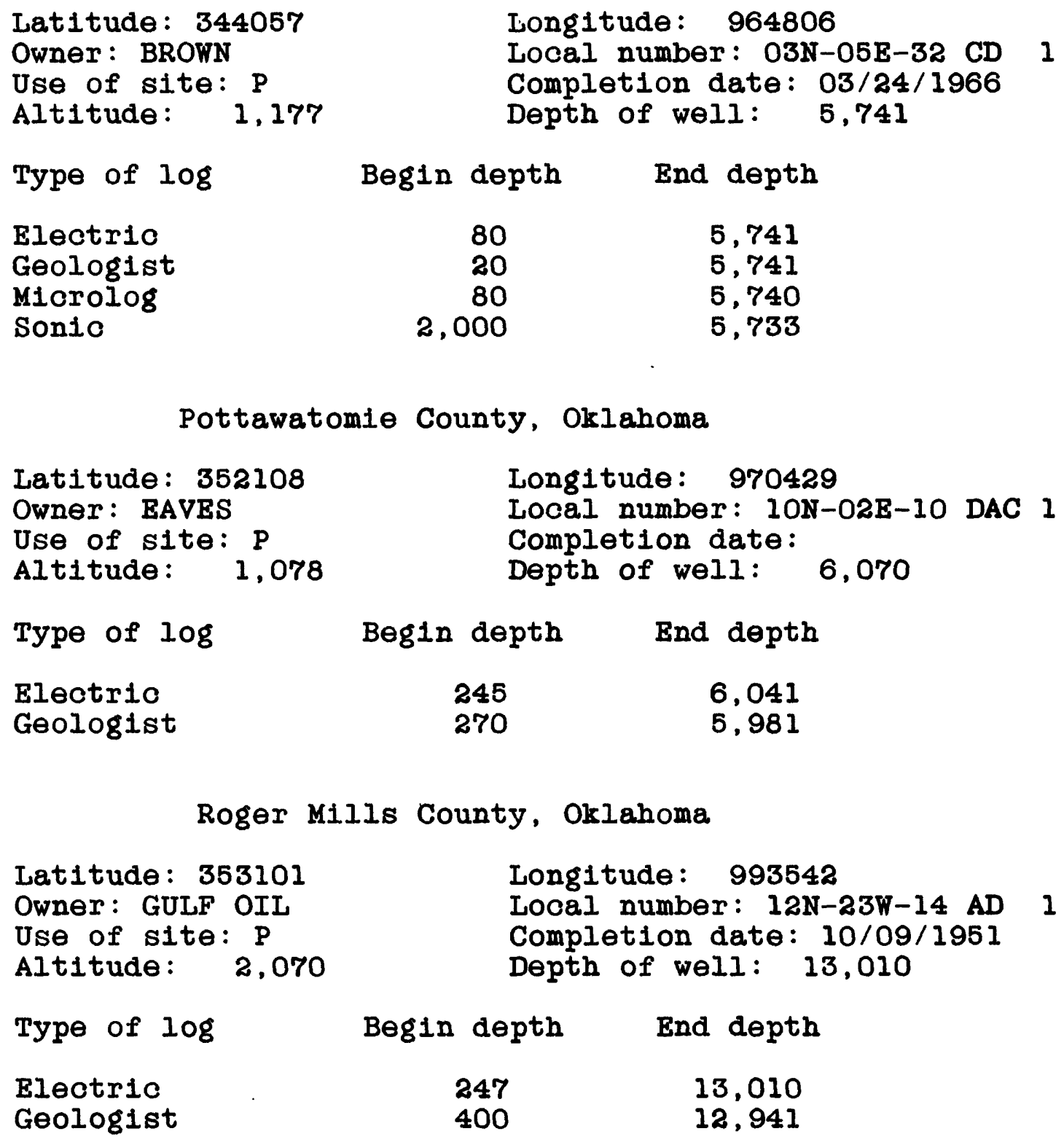


Table 4.--Geophysical logs for wells in the study area--Continued

Roger Mills County, Oklohome

Lat1tude: 354737

Owner: CLARK CNDN

Use of site: $P$

Altitude: $\quad 2,140$

Type of $\log$

Caliper

Electrio

Geologist

Gamma

Neutron

Sonic

Other
Long1tude: 993343

Local number: 15N-22W-08 BDA 1

Completion date: 05/17/1972

Depth of well: 19,435

Begin depth End depth

$\begin{array}{rr}7,700 & 19,435 \\ 4,230 & 19,435 \\ 4,240 & 19,435 \\ 7,700 & 19,435 \\ 12,910 & 19,435 \\ 4,230 & 19,435 \\ 7,700 & 19,435\end{array}$

Seminole County, Oklahoma

Latitude: 351022

Owner: LIVINGSTON Use of site: P

Altitude : 865
Longitude: 963936

Local number: 08N-06E-15 ABB 1 Completion date:

Depth of well: 4,880

Type of $\log$

Begin depth

End depth

Geologist 490 4,880

Sequoyah County, Oklahoma

Latitude: 352757

Owner: SNOW

Use of site: P

Altitude:

Type of 108

Electric

Geologist

Microlog
Longitude: 945526

Local number: 12N-22E-36 CD 1 Completion date: 12/03/1959

Depth of well: 1,718

524

Begin depth

117

130

140
End depth

1,714

1,718

1,716 
Table 4.--Geophysical logs for wells in the study area--Continued

Sequoyah County, Oklahoma

Latitude: 352958

Owner: CHEEK

Use of site: $P$

Altitude:

Type of 108

Electric

Geologist

Microlog 595
Long1tude: 944135

Local number: 12N-25E-19 CAA 1

Completion date: 12/11/1958

Depth of well: 3,504
Begin depth

126

170

600
End depth

$$
\begin{aligned}
& 3,503 \\
& 3,500 \\
& 3,503
\end{aligned}
$$

Stephens County; Oklahoma

Lat1tude: 343533

Owner: FERGUSON

Use of site: P

Alt1tude: $\quad 1,105$

Type of $\log$

Caliper

Electric

Geolog1st

Gamma

Sonic

Other
Longitude: 980323

Local number: 01N-08W-05 AA 1

Completion date: 12/05/1971

Depth of well: 9,152

Begin depth End depth

795

795

0
795

795

795
9,152
9,152
9,150
9,152
9,152
9,152

Stephens County, Oklahoma

Latitude: 342743

Owner: KERR MCGEE

Use of site: $P$

Alt1tude: $\quad 1,133$

Type of 108

Electric

Geolog1st

Gamma

Miorolog

Sontc
Longitude: 980419

Locel number: 01S-08W-19 AA 1

Completion date: 05/31/1966

Depth of well: 8,515
Begin depth End depth

$$
\begin{array}{r}
121 \\
3,000 \\
3,521 \\
1,900 \\
3,521
\end{array}
$$
8,514
8,515
8,505
8,515
8,505 
Table 4.--Geophysical logs for wells in the study area-Continued

\section{Texas County, Oklahoma}

Latitude: 364100

Owner: PHILIIPS

Use of site: T

Altitude: $\quad 3,648$

Type of 108

Geologist

Gamma

Laterolog
Long1tude: 1020015

Local number: 03N-10ECM-32 ACC

Completion date: 05/06/1955

Depth of well: 6.473

Tulsa County, Oklahoma

Latitude: 360619

Owner: RNOCH

Use of site: P

Altitude:

Type of $\log$

Electric

Sonic

822
Begin depth

0
100
100
End depth

6,400

5,429

5,429
Longitude: 960715

Local number: $19 \mathrm{~N}-11 \mathrm{E}-34$ AA 1 Completion date:

Depth of well: $\quad 2,897$
Begin depth

64

400
End depth

$$
\text { 2, } 897
$$

2,897

Wagoner County, Oklahoma

Lat1tude: 365604

Owner: LOVETT

Use of site: $P$

Altitude: 630
Long1tude: 953619

Local number: 17N-16E-22 DBD 1

Completion date: 06/09/1934

Depth of well: 1,600

Type of $\log$

Begin depth

End depth

Geologist

0 1,600

Wagoner County, Oklahoma

Latitude: 355413

Owner: SHELLY

Use of site: $P$

Altitude:

565
Long1tude: 953800

Local number: 17iN-16E-32 CCA 1

Completion date: $12 / 22 / 1980$

Depth of well: 1,681
Type of $\log$

Induction

Gamma-gamma
Begin depth

140

750
End depth

$$
1,580
$$$$
1,580
$$ 
Table 4.--Geophysical logs for wells in the study area--Continued

Washington County, Oklahoma

Latitude: 365825

Owner: THOMASON

Use of site: $P$

Altitude: $\quad 750$

Type of $\log$

Geologist
Longitude: 955250

Local number: 29N-13E-22 DDB 1 Completion date: 02/03/1917

Depth of well: 3,175

\section{Begin depth End depth}

$0 \quad 3,175$

Washita County, Oklahoma

Latitude: 350723

Owner: NO. AMER.

Use of site: $P$

Alt1tude: 1,602

Type of 108

\section{Caliper}

Electric

Geologist

Gamma

Neutron

Sonic

Other

Microlog
Longitude: 990353

Local number: 08N-18W-35 DAB 1 Completion date:

Depth of well: 15,013
Begin depth

10,306
294
200
10,306
600
600
10,302
10,306
End depth

$$
\begin{array}{r}
14,893 \\
15,010 \\
15,013 \\
14,893 \\
1,913 \\
14,996 \\
14,890 \\
14,893
\end{array}
$$

Woods County, Oklahoma

Lat1tude: 364257

Owner: ATLANTIC

Use of site: T

Altitude: 1,465

Type of $\mathrm{log}$

Electric

Geologist
Long1tude: 984616

Local number: $26 \mathrm{~N}-15 \mathrm{~W}-24 \mathrm{CB}$

Completion date: 07/31/1948

Depth of well: 7,370

Begin depth End depth

389

410
7,271

7,370 
Table 4.--Geophysical logs for wells in the study area--Continued

Woodward County, Oklahoma

Latitude: 362941

Owner: SINCLAIR

Use of site: T

Altitude: 2,010

Type of 108

Electric

Geologist
Longitude: 991557

Local number: 23N-19W-06 DD

Completion date: 05/07/1951

Depth of well: 10,327

Begin depth End depth

$\begin{array}{rr}3,167 & 10,310 \\ 120 & 10,327\end{array}$

Carson County, Texas

Latitude: 352030

Owner: WHITTENBURG

Use of site: T

Altitude: $\quad 3,500$

Type of 108

Electric

Gamma

Laterolog
Longitude: 1013000

Local number: GIBSON BLK M4 SEC 4 Completion date: 08/01/1953

Depth of well: 9,311

Begin depth End depth
0
9,311
1,061
9,166
1,061
9,166

Dallam County, Texas

Latitude: 360630

Owner: SKELLY

Use of site: T

Altitude: $\quad 4,327$

Type of $\log$

Geologist

Induction

Laterolog

Microlog
Longitude: 1025300

Local number: CSS BLK 5 SEC 58

Completion date: 11/07/1958

Depth of well: 8,085

Begin depth End depth
0
2,937
8,085
2,938
8,084
2,938
8,085
8,085 
Table 4.--Geophysical logs for wells in the study area--Continued

\section{Dallam County, Texas}

Latitude : 361940

Owner: CONTINENTAL OIL Use of site: P

Altitude: $\quad 4,415$

Type of 108

Geologist
Longitude: 1025245

Local number: CSS BLK.81,7

Completion date:

Depth of well: 7,808

Begin depth End depth

1,440

7,808

Dallam County, Texas

Latitude : 362645

Owner: HUMBLE OIL CO. Use of site: $P$

Altitude: $\quad 3,854$

Type of $\log$

Geologist
Longitude: 1021540

Local number: H TC RR BLK. 50,16 Completion date:

Depth of well: 6,214

Begin depth End depth

610

6,214

Gray County, Texas

Latitude: 353030

Owner: PHILLIPS

Use of site: $T$

Altitude: $\quad 2,854$

Type of 108

Caliper

Geologist

Induction

Gamma

Laterolog

Neutron

Sonic

Other
Longitude: 1003730

Local number: H GN;BLK A-6 SEC 53 Completion date: 08/14/1972

Depth of well: 13,594

Begin depth End depth

3,677

13,583

13,594

13,578

13,583

13,578

13,583

13,575

13,583 
Table 4.--Geophysical logs for wells in the study area--Continued

\section{Hansford County, Texas}

Latitude: 361760

Owner: GULF OIL Use of site: T Altitude: $\quad 3,193$

Type of 108

Caliper

Geologist

Induction

Gamma

Laterolog
Longitude: 1012400

Local number: H GN;BLK P, SBC 17 Completion date: 07/17/1968

Depth of well: $\quad 9,440$

Begin depth End depth

$\begin{array}{rr}0 & 9,438 \\ 0 & 9,432 \\ 2,985 & 9,436 \\ 0 & 9,438 \\ 2,985 & 9,436\end{array}$

Hartley County, Texas

Lat1tude: 354400

Longitude: 1024900

Owner: SHAMROCK OIL CO. LOCal number: G M, LE, 7 Use of site: $P$

Altitude: $\quad 4,031$

Completion date:

Depth of well: 6,114

Type of $\log$

Begin depth

End depth

Geologist

1,305

6,114

Hartley County, Texas

Latitude: 355330

Owner: PHILLIPS

Use of site: T

Altitude: $\quad 3,955$

Type of 108

Blectric

Geologist

Microlog
Long1tude: 1023500

Local number: T NO;BLK ITO SEC 18

Completion date: $12 / 02 / 1955$

Depth of well: $\quad 9,166$

Begin depth Bnd depth

$\begin{array}{rr}468 & 9,166 \\ 0 & 9,150 \\ 3,990 & 5,995\end{array}$


Table 4.--Geophysical logs for wells in the study area--Continued.

\section{Hemphill County, Texas}

Lat1tude: 354130

Owner: PHILLIPS

Use of site: T

Altitude: $\quad 2,488$

Type of 108

Callper

Geologist

Induction

Gamma

Sonic

Other
Long1tude: 1000530

Local number: G MMB A; BLK C,S 255 Completion date: 08/26/1967

Depth of well: 20,113
Begin depth

4,020

4,020

4,020

100

4,020
End depth

$$
\begin{aligned}
& 16,703 \\
& 20,100 \\
& 20,103 \\
& 16,703 \\
& 20,105 \\
& 20,110
\end{aligned}
$$

Hutchinson County, Texas

Lat1tude: 360100

Owner: SUN COMPANY

Use of site: $T$

Altitude: 3,154

Type of $\log$

Caliper

Electric

Geologist
Long1tude: 1010900

Local number: GB CNG; BLK R; SEC 77

Completion date: $01 / 17 / 1958$

Depth of well: 8,287

Begin depth End depth
2,709
2,709
8,282
8,285
0
8,287

\section{Hutchinson County, Texas}

Lat1tude: 353930

Owner: TEXAS OIL GAS

Use of site: P

Alt1tude: 3,111

Type of $\log$

Geologist
Long1tude: 1013545

Local number: H TC RR, 46, 89

Completion date:

Depth of well: 5,457

Begin depth End depth

1,500

5,457 
Table 4.--Geophysical logs for wells in the study area--Continued

Lipscomb County, Texas

Latitude: 361130

Owner: GULF OIL

Use of site: $T$

Altitude: 2,785

Type of $\log$

Electric

Geologist

Gamma
Longitude: 1003000

Local number: H TCRR;BLK 43;S 413

Completion date: 12/08/1949

Depth of well: 14,278

$$
\text { Begin depth End depth }
$$

$\begin{array}{ll}306 & 13,494 \\ 120 & 14,278 \\ 306 & 13,494\end{array}$

Moore County, Texas

Latitude: 355630

Owner: SAC-MOBILE

Use of site: $T$

Altitude: $\quad 3,416$

Type of $10 \mathrm{~g}$

Caliper

Geologist

Induction

Gamma

Sonic
Longitude: 1014300

Local number: T NO,BLK 3T, SEC 183 Completion date:

Depth of well: 6,987

Begin depth End depth

Ochiltree County, Texas

Latitude: 360530

Owner: GULF OIL

Use of site: $T$

Altitude: 3,077

Type of $\log$

Electric

Geologist
Longitude: 1005730

Local number: J.J.BALINTINE SURVEY

Completion date: 01/25/1950

Depth of well: 11,640

$$
\text { Begin depth End depth }
$$

927

11,632

0 
Table 4.--Geophysical logs for wells in the study area--Continued

\section{Oldham County, Texas}

Lat1tude: 353500

Owner : SHELL

Use of site: $T$

Altitude: 3,496

Type of 108

Geologist

Gamma

Laterolog

Microlog

Sonic
Longitude: 1021200

Local number: G M;BLK 2;SEC 64

Completion date: $02 / 16 / 1960$

Depth of well: 12,964

Begin depth End depth

$\begin{array}{rr}85 & 12,964 \\ 20 & 12,960 \\ 110 & 12,960 \\ 38 & 9,296 \\ 10,602 & 12,958\end{array}$

Oldham County, Texas

Latitude: 352315

Owner: SUPERIOR OIL CO Use of site: $P$

Altitude: $\quad 3,861$

Type of 108

Geologist
Longitude: 1024240

Local number: LGE 31 CAPITAL LANDS

Completion date:

Depth of well: 5,499

$$
\text { Begin depth End depth }
$$

750

5,499

\section{Oldham County, Texas}

Latitude: 351130

Owner: LIVERMORE INC.

Use of site: $P$

Altitude: 3,881

Type of $\log$

Geologist
Longitude: 1025715

Local number: $7 \mathrm{~N}-1 \mathrm{E}$, SEC. 26

Completion date:

Depth of well: 6,884

Begin depth End depth
2,170
6,884 
Table 4.--Geophysical logs for wells in the study area--Continued

\section{Potter County, Texas}

Latitude: 352100

Owner: WHITTENBURG

Use of site: $T$

Altitude: $\quad 3,568$

Type of $\log$

Geologist

Gamma

Laterolog
Long1tude: 1014030

Local number: BS F; BLK 1; SEC 28

Completion date: $01 / 30 / 1955$

Depth of well: 12,581

Begin depth End depth

$\begin{array}{rl}30 & 12,581 \\ 586 & 12,194 \\ 586 & 12,194\end{array}$

Roberts County, Texas

Latitude: 354130

Owner: PENNZOIL

Use of site: T

Altitude: $\quad 2,795$

Type of 108

Caliper

Geologist

Induction

Gamma

Sonic

Other
Longitude: 1003730

Local number: H GN;BLK M2; SEC 62 Completion date: 08/29/1974

Depth of well: 14,552

Begin depth End depth

$\begin{array}{rr}4,887 & 14,167 \\ 30 & 14,552 \\ 790 & 14,164 \\ 790 & 14,164 \\ 4,887 & 14,167 \\ 4,887 & 14,187\end{array}$

Sherman County, Texas

Latitude: 362230

Owner: PHILLIPS

Use of site: T

Altitude: $\quad 3,448$

Type of $\log$

Geologist

Induction

Laterolog

Microlog

Other
Longitude: 1014430

Local number: GH H;BLK 1C, SEC 68

Completion date: $01 / 13 / 1960$

Depth of well: 8,210

\section{Begin depth End depth}

$\begin{array}{rr}0 & 8,210 \\ 2,101 & 7,702 \\ 2,101 & 7,702 \\ 2,101 & 7,702 \\ 3,000 & 8,210\end{array}$


Table 4.--Geophysical logs for wells in the study area--Continued

\section{Sherman County, Texas}

Latitude: 360700

Owner: I.T.I.O.

Use of site: $P$

Altitude: $\quad 3,639$

Type of 108

Geolog1st
Longitude: 1020130

Local number: T NO RR, IT, 369

Completion date:

Depth of well: 5,138

Begin depth End depth

1,400

6,138

Wheeler County, Texas

Lat1tude: 352100

Owner: STD TEXAS

Use of site: T

Altitude: $\quad 2,197$

Type of $\log$

Caliper

Geologist

Laterolog

Sonic

Other
Longitude: 1000500

Local number: H GN;BLK A7, SEC 36 Completion date: 10/02/1970

Depth of well: 21,648

Begin depth End depth

$\begin{array}{rr}380 & 21,647 \\ 385 & 21,640 \\ 380 & 21,642 \\ 380 & 21,647 \\ 4,410 & 21,647\end{array}$

Union County, New Mexico

Latitude: 360140

Owner: OIL EXPLORATION Use of site: P

Altitude: $\quad 4,526$

Type of $\log$

Electric

Geologist
Long1tude: 1030745

Local number: 21N-36E-29 BB

Completion date: $01 / 31 / 1956$

Depth of well: 7,360
Begin depth End depth

153

750
7,354

7,360 
Table 4.--Geophysical logs for wells in the study area--Continued

\section{Union County, New Mexico}

Latitude: 362015

Owner: CONTINENTAL OIL

Use of site: P

Altitude: $\quad 4,678$

Type of 108

Geologist

Gamma

Neutron
Longitude: 1030320

Local number: 24N-36E-2-DD

Completion date: 04/04/1957

Depth of well: 5,308

Begin depth End depth

5,308

$80 \quad 5,308$

$80 \quad 5,308$

Union County, New Mexico

Latitude: 362745

Owner: HOXSEY OIL CO.

Use of site: $P$

Altitude: $\quad 5,509$

Type of $\log$

Electric

Geologist
Longitude: 1033030

Local number: 26N-32E-27 AD

Completion date: 08/01/1956

Depth of well: 3,898

Begin depth End depth

$203 \quad 3,521$

$290 \quad 3,898$

Union County, New Mexico

Latitude: 364438

Owner: TEXACO 1 CRUZ

Use of site: T

Altitude: $\quad 4,835$

Type of 108

Electric

Induction

Laterolog
Longitude: 1030408

Local number: 29N-36E-14 CCA 1

Completion date: 02/05/1964

Depth of well: 6,460
Begin depth End depth

$\begin{array}{rr}20 & 6,460 \\ 431 & 6,448 \\ 431 & 6,448\end{array}$

Union County, New Mexico

Latitude: 364710

Owner: NELSON MOORE

Use of site: $T$

Altitude: $\quad 6,710$

Type of $10 \mathrm{~g}$

Geologist
Longitude: 1035110

Local number: $30 \mathrm{~N}-29 \mathrm{E}-33$ DCC Completion date: 07/25/1951

Depth of well: 2,685
Begin depth End depth

0 2,685 
Table 4.--Geophysical logs for wells in the study area--Continued

\section{Union County, New Mexico}

Latitude: 365545

Owner: GULF OIL CO

Use of site: $P$

Alt1tude: $\quad 5,300$

Type of $\log$

Electric

\section{Longitude: 1032650}

Local number: $31 \mathrm{~N}-33 \mathrm{E}-17 \mathrm{BC}$

Completion date: 03/08/1970

Depth of well: 2,855
Begin depth

333
End depth

2,828 